\title{
Session 2aAA
}

\section{Architectural Acoustics and Engineering Acoustics: Loudspeaker Student Design Competition}

\author{
Neil A. Shaw, Cochair \\ Menlo Scientific Acoustics, Inc., P.O. Box 1610, Topanga, California 90290-1610 \\ Allan Devantier, Cochair \\ Infinity Engineering, Harman Consumer Group, 8500 Balboa Boulevard, Northridge, California 91329

\begin{abstract}
The ASA Technical Committees on Engineering Acoustics and Architectural Acoustics, in association with the American Loudspeaker Association, JBL Professional, JBL Consumer, Infinity and Revel, are sponsoring a Student Design Competition, which will be professionally judged at this meeting. The purpose of this design competition is to encourage students enrolled in Engineering, Physics, and other University curriculums that involve engineering, physics, materials, electroacoustics and/or acoustics to express their knowledge of electroacoustic transducer design. This knowledge will be demonstrated in the prototype design of an electroacoustic transduction system where various design parameters must be balanced to provide a product that meets the design objectives. The 2000 competition involves the design of a loudspeaker system, one specimen of which is to be developed, that may be placed on a pedestal or surface mounted to a room surface with physical and performance characteristics defined by the Design Specification.
\end{abstract}

The submitted designs will be judged by a panel of professional loudspeaker designers and design consultants at the meeting. An award of $\$ 1,000$ US will be made to the submitter(s) of the entry judged "First Honors." Four awards of $\$ 500$ US each will be made to the submitters of entries judged "Commendation."

Entries will be on a display in this session. Each entry consists of a poster presentation and a prototype specimen device. In addition, the results of the objective and subjective tests performed for each entry will also be presented.

Session 2aAO

\section{Acoustical Oceanography: Bioacoustics III}

\author{
Timothy K. Stanton, Chair \\ Department of Applied Ocean Physics and Engineering, Woods Hole Oceanographic Institution, Bigelow 20,
} Woods Hole, Massachusetts 02543-1053

Chair's Introduction-7:50

\section{Contributed Papers}

8:00

2aAO1. Acoustic investigation of the Hawaiian mesopelagic boundary community. Kelly Benoit Bird and Whitlow Au (Hawaii Inst. of Marine Biol., 46-700 Lilipuna Rd., Kaneohe, HI 96744)

A $200 \mathrm{kHz}$ echosounder modified to sample directly into a computer was used to survey the mesopelagic boundary community (MBC) in waters off the leeward coasts of three Hawaiian Islands from dusk to dawn. The target strengths of individual mesopelagic organisms captured in Isaacs-Kidd midwater trawls were measured with the echosounder in a shipboard seawater tank for use in echo-energy integration calculations. Acoustic survey data were mapped in ArcView, allowing analysis of spatial structure. The MBC is spatially heterogeneous both horizontally and vertically. The parameters of patch structure vary significantly throughout a night and between islands. The density of mesopelagic organisms was consistently higher off the coasts of Oahu and Lanai than the coast of Hawaii. However, the density of organisms observed in all locations was high, reaching a maximum of 1800 organisms $/ \mathrm{m}^{3}$ off Oahu and 700 organisms $/ \mathrm{m}^{3}$ off Hawaii. Patterns in density were significantly affected by the location's distance from the shoreline, but not by its depth. Patterns in density suggest that the MBC undergoes a significant diel horizontal migration in addition to its well-documented vertical migration. Due to this predictable and conserved horizontal migration, the $\mathrm{MBC}$ is found much closer to shore than previously reported.

\section{8:15}

2aAO2. Acoustical study of the prey field and supporting food web for whales in the Ligurian Sea north of Corsica. Duncan E. McGehee (BAE Systems, 4669 Murphy Canyon Rd., Ste. 102, San Diego, CA 92123, duncan.mcgehee@baesystems.com) and David A. Demer (Southwest Fisheries Sci. Ctr., La Jolla, CA 92037)

The Sound, Oceanography, and Living Marine Resources (SOLMAR) program is a study coordinated by the NATO SACLANT Undersea Research Centre to develop ways of mitigating risks to whales from anthropogenic sound sources. One objective of the SOLMAR program is to 
examine the correlation between the distribution of whales in parts of the Mediterranean Sea and that of their prey, lower trophic levels, and basic oceanographic parameters. A multiship field study was conducted in the Ligurian Sea north of Corsica in August 1999, as will be a follow-up study in August 2000. The primary role of the R/V Ammiraglio Magnaghi is to map basic physical oceanographic parameters with a CTD; phytoplankton with a fluorometer; small zoo-plankton (copepods and smaller) with a six-frequency TAPS (Tracor Acoustic Profiling System) and small plankton net; and large zooplankton (euphausiids and larger) with a $120-\mathrm{kHz}$ split-beam echosounder. Measurements in 1999 were consistent with a counterclockwise circulation in the basin causing Ekman pumping of nutrients in the center. Phytoplankton were concentrated in the center, small zooplankton were around the periphery, and larger zooplankton were associated with the shelf break and basin center. Results from the two field seasons will be discussed and compared.

\section{$8: 30$}

2aAO3. Biophysical interactions at the Almeria-Oran front. Sophie Fielding, Nick Crisp, and Alex Mustard (Southampton Oceanogr. Ctr., European Way, Southampton SO14 3ZH, UK)

The Almeria-Oran front forms where Atlantic origin waters meet Mediterranean surface waters at the eastern end of the Alboran Sea. Following a multidisciplinary cruise on RRS DISCOVERY in December 1996, analysis of temperature and salinity on density surfaces at the front showed that periodic subduction of Mediterranean surface waters (MSW) influenced the distribution of phytoplankton and zooplankton in the frontal region. A layer of fluorescence coincident with the subducted MSW indicated that phytoplankton were drawn down and along isopycnals to depths of $200 \mathrm{~m}$. The study of scattering layers, identified with acoustic backscatter data from the shipboard ADCP and a SIMRAD EK500 echosounder, revealed that a layer of zooplankton was also found coincident with the drawn-down phytoplankton. This layer persisted during and despite diel vertical migration. Smaller zooplankton, not undertaking diel vertical migration, were concentrated in the fast-flowing frontal jet as seen in highresolution OPC data. EK500 target-strength data indicate that the subducted communities may constitute different size classes to those in the surrounding waters. Samples from Longhurst Hardy plankton recorder tows across the front provide additional ground truthing for our acoustic observations. [Work supported by EU MAST, NERC, and DERA.]

\section{$8: 45$}

2aA04. Changes in volume reverberation from deep to shallow water in the Gulf of Mexico. Richard H. Love (BayouAcoust., 209 Baywood Dr., Pass Christian, MS 39571), Charles H. Thompson, and Redwood W. Nero (Stennis Space Center, MS 39529)

Volume reverberation at frequencies below $10 \mathrm{kHz}$ is caused primarily by resonance scattering from swimbladders of dispersed fish. Since fish populations change from deep to shallow water, the character of volume reverberation should likewise change. However, there are few data available from shelf and slope waters to document expected changes. Therefore, the Naval Research Laboratory conducted a short experiment to examine volume reverberation in deep, slope, and shelf waters of the eastern Gulf of Mexico. Day and night measurements at frequencies from 1 to 10 $\mathrm{kHz}$ were made with shallow explosive sources and downward-looking receivers in water depths of 200, 450, and over $3000 \mathrm{~m}$. Reverberation at the deep location was typical of that caused by small mesopelagic fishes of the 'deep scattering layer;', scattering strengths increased rapidly with frequency up to about $5 \mathrm{kHz}$ and were relatively constant at higher frequencies. At the shelf location, scattering strengths were low during the day and increased by $20 \mathrm{~dB}$ at $10 \mathrm{kHz}$ to $40 \mathrm{~dB}$ below $2 \mathrm{kHz}$, as larger fishes rose off bottom at night. Reverberation over the slope combined characteristics of the deep and shelf locations. [Work supported by ONR.]
2aAO5. Herring hydroglyphics in littoral waters of the northern Gulf of Mexico. Redwood W. Nero, Charles H. Thompson, John R. Dubberley (Naval Res. Lab., Stennis Space Center, MS 39529-5004, woody.nero@nrlssc.navy.mil), and Richard H. Love (Bayou Acoust., Pass Christian, MS 39571-2111)

A large shoal of fish occurring in the vicinity of the 220-m isobath was observed using a standard $38-\mathrm{kHz}$ fisheries echosounder and a $1.5-$ to $10-\mathrm{kHz}$ low-frequency fish sonar (LFFS) for several days in July 2000. The fish behaved like herring, exhibiting a rapid rise to the sea surface at dawn, formation into schools, and a rapid descent to the sea floor. Schools remained at depth through the day and gradually rose to the sea surface at dusk and then rapidly descended and spread out into a diffuse scattering layer at 75- to $125-\mathrm{m}$ depth. Shifts in resonance frequencies during migration, release of gas bubbles during migration, and strong avoidance of the vessel when maneuvering, all suggest the fish were most likely round herring, Etrumeus teres, which are common at these depths in the NMFS historical trawl survey data. An examination is made of some of the scattering characteristics of the schools and layers of these fish and comparisons of the $38-\mathrm{kHz}$ data to scattering at $500-\mathrm{Hz}$ bands from 1.5 to $5 \mathrm{kHz}$ and $1-\mathrm{kHz}$ bands from 5 to $10 \mathrm{kHz}$. [Work supported by ONR.]

\section{9:15}

2aAO6. Monitoring fish movement using an ADCP. Ranjan Patro, Len Zedel, and Cristina Spanu-Tollefsen (Dept. of Phys. and Physical Oceanogr., Memorial Univ. of New Foundland, St. John's, NF A1B 3X7, Canada)

An Acoustic Doppler Current Profiler, ADCP, can detect the presence of fish in water using the backscatter intensity. The Doppler profiler does not, however, make a point measurement; rather, measurement is made by averaging multiple beams and assuming that the current velocity is uniform between the distinct sample locations. As a result, individual fish speed cannot be measured. However, data are presented that demonstrate that the ADCP can measure the swimming speed of large fish schools. Fish speed and direction were measured for Norwegian spring herring. Observed speeds were $0-40 \mathrm{~cm} \mathrm{sec}^{-1}$. Diel vertical migrations were observed with Norwegian herring ascending to the surface at dusk, and descending to greater depth at dawn. The accuracy and precision of an ADCP is a complex function of many variables (i.e., frequency, pulse length, transducer characteristics, backscatter strength, type and distribution of scatters). The other problems to be considered are sampling criteria and calibration. These sampling problems are explored for the case of ADCP measurements of fish movement. [Work supported by the Natural Sciences and Engineering Research Council of Canada and an Atlantic Canada Opportunities Agency Infrastructure grant.]

\section{$9: 30$}

2aA07. Comparison of measured and calculated absorption spectra due to ensembles of fish with swim bladders. Orest Diachok (Naval Res. Lab., Washington, DC 20375)

Absorption spectra derived from long-term, broadband transmission loss measurements were compared with calculated absorption spectra due to ensembles of identical fish with swim bladders over the frequency range 0.5-5.0 kHz, and near-coincident trawling data. The dominant fishes during this experiment were sardines $(\sim 60 \%)$ and anchovies $(\sim 30 \%)$. Only nighttime data, when the separation between these fishes is large (compared to wavelength), were considered. Measured distributions of the lengths of sardines and anchovies were separated into groups, which were of nearly uniform length-essentially year classes. Matching of measured and calculated absorption spectra yielded estimates of the number densities and $Q$ 's of species and year classes. The spatial number densities of adult sardines, which were derived from absorptivity measurements $\left(1.1 / \mathrm{m}^{2}\right)$ and nearly coincident echo sounder data $\left(0.6-1.6 / \mathrm{m}^{2}\right)$, were consistent. The $Q$ 's and resonance frequencies of the absorption lines, which were attributed to adult and juvenile sardines, were approximately 
2.3 at $1.3 \mathrm{kHz}$ and 4.7 at $3.8 \mathrm{kHz}$, respectively. These values of $Q$ appear to be dominated by the $Q_{0}$ of individual fish. Limited laboratory measurements of $Q_{0}$ of other species versus fish age are consistent with the observed age dependence. [Work supported by ONR.]

\section{9:45}

2aA08. Species identification of pelagic fish schools using acoustic descriptors and ancillary information. Gareth Lawson ${ }^{\text {a) }}$ (Marine and Coastal Management, Private Bag X2, 8012 Rogge Bay, South Africa), Manuel Barange (Plymouth Marine Lab., Plymouth PL1 3DH, UK), and Pierre Freon (Institut de Recherche pour le Developpement, France)

It is shown that schools of similarly sized and behaving pelagic fish species can be accurately identified to species over a large spatial area, on the order of the broad spatial-scales typical of standard assessment surveys, and over a time-span of three years. Acoustic measurements of morphometric, energetic, and bathymetric features of anchovy, sardine, and round herring schools were extracted using commercially available software from acoustic data collected by a conventional single-frequency narrow-band echosounder during trawling operations of pelagic stock surveys of the South African continental shelf (November 1997, 1998 and 1999). Discriminant function analysis of 18 descriptors of the 214 schools for which the species composition was satisfactorily determined by trawl samples indicated that schools could be correctly identified to species in $88.3 \%$ of all cases $(94.9 \%$ for anchovy, $82.6 \%$ sardine, $82.6 \%$ round herring). The three species were differentiated primarily on the basis of school bathymetric position and backscattered energy. Including ancillary information (latitude, longitude, sea surface temperature, bottom depth, and time of day) in analysis improved the accuracy of school identification to $94.9 \%$, since such variables allowed discrimination on the basis of interspecific differences in habitat use, as well as in schooling behavior. a) Currently at MIT/Woods Hole Joint Program.

\section{0:00-10:15 Break}

\section{0:15}

2aA09. Estimating the scattering contribution from microstructure and marine organisms using multiple-frequency acoustic data collected from a Gulf of Maine internal wave. Joseph D. Warren, Peter H. Wiebe (Woods Hole Oceanogr. Inst., Woods Hole, MA 02543), Harvey E. Seim (Univ. of North Carolina, Chapel Hill, NC 27599), and Timothy K. Stanton (Woods Hole Oceanogr. Inst., Woods Hole, MA 02543)

High-frequency sound $(>10 \mathrm{kHz})$ is scattered in the ocean by many different sources. Marine organisms are often thought to be the primary source of backscattered sound energy. Because of this, acoustical methods are used widely to quickly survey large areas of the ocean for the distribution and abundance of animals. Field experiments and recent theoretical work suggest that temperature and salinity microstructure in some oceanic regions could cause acoustic scattering at levels comparable to that caused by organisms. A method which uses multiple-frequency acoustic data to discriminate between biological and physical sources of scattering has been developed and applied to data collected in an internal wave in the Gulf of Maine. Model calculations show that in certain regions, scattering contributions from turbulence are equal to the contributions from animals. Using net tow information to account for the scattering from biological sources, acoustic scattering data combined with temperature and salinity profiles are inverted for estimates of the dissipation rate of turbulent kinetic energy $(\epsilon)$. The estimates of $\epsilon$ made at the depths where an internal wave occurred are several orders of magnitude higher than the $\epsilon$ estimates from depths above and below the internal wave.
10:30

2aAO10. Comparison and evaluation of methods for distinguishing among several functional classes of zooplankton and fish using acoustic backscatter data at four frequencies. Gordon L. Swartzman (Appl. Phys. Lab., Univ. of Washington, Seattle, WA 98105), D. Van Holliday (BAE SYSTEMS, San Diego, CA 92123), Kenneth O. Coyle (Univ. of Alaska, Fairbanks, AK), and Jeffrey M. Napp (Alaska Fisheries Sci. Ctr., Seattle, WA 98115)

Zooplankton net samples data were collected at 30 locations near the Pribilof Islands, Alaska in September 1999 using a MOCNESS. Simultaneous volume backscatter data were collected with an HTI echosounder system at: 43, 120, 200, and $420 \mathrm{kHz}$. Three algorithms were compared for their ability to classify acoustic data into the dominant zooplankton functional/size groups (euphausiids, copepods, and ostra cods) and separating them from fish. The algorithms tested include: (1) combining morphological image processing and image differences to identify patches in different size ranges, and using the forward problem calibrated to plankton found in MOCNESS hauls to ascribe size/functional groups to the different patches; (2) application of canonical correlation between acoustics (backscatter volume and target strengths) and plankton biomass captured by the MOCNESS; and (3) application of inverse techniques to estimate the number of individuals in set size classes using multiple theoretical models of backscatter volume compared to plankton biomass captured by a net. Knowledge of the strengths and weaknesses of each technique allows us to better interpret broad-scale acoustic survey results from the same cruise, thus providing a synoptic view of the fish and their plankton prey which are in the size and density range to be acoustically detected.

\section{0:45}

2aAO11. Validity study of the distorted wave Born approximation (DWBA) model: Application to the acoustic scattering by marine organisms. Dezhang Chu and Timothy Stanton (Dept. of Appl. Phys. and Eng., Woods Hole Oceanogr. Inst., Woods Hole, MA 02543)

Applications of the distorted wave Born approximation (DWBA) to the acoustic scattering by weakly scattering marine organisms have shown much progress [Chu et al., J. Acoust. Soc. Am. 93, 2985-2988 (1993); Stanton and Chu, ibid. 103, 236-253 (1998)]. Although the applicability of the model has been examined for simple objects such as spheres, cylinders, and prolate spheroids, the validity of applying the DWBA model to the acoustic scattering by marine organisms with more complicated shapes has not been studied. To evaluate the performance of the DWBA model, a systematic study of the DWBA will be presented. An in-depth study of the 1D problem reveals the fundamental difference between the exact and DWBA solutions and helps us better understand the scattering problem. By studying the internal field of $1 \mathrm{D}, 2 \mathrm{D}$, and $3 \mathrm{D}$ problems for a more general bistatic geometry and evaluating the volume integral derived from the exact Helmholtz-Kirchhoff integral equation, we are able to estimate the validity of the DWBA model in describing the acoustic scattering by arbitrarily shaped marine organisms. [Work supported by NSF and ONR.]

\section{1:00}

2aAO12. Wavelet analysis comparing spectral characteristics of high frequency acoustics and hydrographic properties on Georges Bank. Karen Fisher (2154 Snee Hall, Cornell Univ., Ithaca, NY 14853, kef10@cornell.edu) and Peter Wiebe (Woods Hole Oceanogr. Inst., Woods Hole, MA 02543)

Acoustic surveys of Georges Bank were carried out during the GLOBEC Broadscale program in January, March, and June of 1998 and 1999. The Greene Bomber towfish collected temperature, salinity, and fluorescence as well as the acoustics data at 120 and $420 \mathrm{kHz}$, from approximately 3 meters depth on all six cruises. With over 1200 kilometers of acoustic trackline on each cruise, covering well mixed, frontal, and well stratified water columns, a method is needed to characterize patch structures encountered. Wavelet analysis offers a computationally effective approach that can be applied to all the data obtained along the trackline. 
Variance analysis of the wavelet transform provides both spatial and spectral resolution simultaneously, allowing distinct consideration of spectra obtained in any spatial subset of the entire dataset. Temporal and spatial variations in the characteristic slopes and length scales of temperature, salinity, and fluorescence indicate that distinct spectral signatures exist that can be exploited to sort out physical and biological contributions. The extent to which physical and biological spectral signatures influence the wavelet variance spectra of the acoustic data is examined in each hydrographic regime.

\section{$11: 15$}

2aA013. Volume acoustic backscattering measurements with a 68$\mathbf{k H z}$ cylindrical array. Timothy C. Gallaudet and Christian P. de (Marine Physical Lab., Scripps Inst. of Oceanogr., UCSD (Code 0205), 9500 Gilman Dr., La Jolla, CA 92037-0205, tcg@mpl.ucsd.edu) Moustier
Most sensors designed to measure volume acoustic backscatter over the entire water column have limited horizontal coverage. The $68 \mathrm{-kHz}$ cylindrical arrays of the U.S. Navy's Toroidal Volume Search Sonar (TVSS), towed $80 \mathrm{~m}$ below the sea surface in 200-m water depth, have been used to measure volume backscatter over the entire water column with about $4.5 \mathrm{deg}$ of angular resolution over a swath roughly $300 \mathrm{~m}$ wide. The data were analyzed to determine the volume acoustic backscattering strengths and cross sections, and the target strengths of (1) near-surface bubble clouds in the tow ship wake; (2) midcolumn zooplankton layers; and (3) near-bottom fish schools. Unlike previous acoustic studies of ship wakes, these results are obtained from data which sample the bubble field at a constant distance from the ship. Echo integration over a midcolumn scattering layer is used to assess the patchiness of zooplankton in the region. Target strengths of near-bottom fish schools are easily distinguished from the surrounding seafloor backscattering strengths. [Work sponsored by ONR-NRL (Contract No. N00014-96-1-G913).]

11:30-12:00

Panel Discussion

\title{
Session 2aBB
}

\section{Biomedical Ultrasound/Bioresponse to Vibration, Physical Acoustics and Acoustical Oceanography: Detection and Characterization of Bubbles, Acoustic Cavitation, and Associated Physical Effects I}

\author{
Ronald A. Roy, Cochair \\ Department of Aerospace and Mechanical Engineering, Boston University, 110 Cummington Street, \\ Boston, Massachusetts 02215 \\ Timothy G. Leighton, Cochair \\ Institute of Sound and Vibration Research, University of Southampton, Highfield, Southampton SO17 1BJ, United Kingdom
}

Chair's Introduction-7:30

Invited Papers

$7: 35$

2aBB1. Methods for detecting bubbles and monitoring cavitation activity; a report from Working Group 22 of Standards Committee S1. Wesley L. Nyborg (Phys. Dept., Univ. of Vermont, Burlington, VT 05405)

Few liquids or solids are homogeneous; most contain cavities filled with air or other gas. They may be large or small, desirable or harmful. In diagnostic medicine, small gas-containing particles are introduced into the circulation as contrast agents to improve ultrasound images. In lakes and oceans, bubbles produced at the surface present difficulties for sound propagation. In industries gaseous phase is sometimes desired in manufactured products, but unwanted gas bubbles can be a serious problem. Exposing cavities to sound produces a complex panoply of activity which includes simple breathing oscillations, shape oscillations, high-speed travel, jet formation, dramatic implosions, fragmentation, bubble growth and microstreaming. Mechanical stresses and chemicals produced by acoustic cavitation are capable of causing a host of physical, chemical and biological effects which can be desired, as in sonic cleaning or sonoporation, or harmful if unwanted chemical action or solid erosion is produced. The ASA Committee on Standards established a working group to prepare a technical report dealing with the many techniques which have been used to characterize bubbles and monitor cavitation activity. In this report, 25 techniques are described. Principles of operation are explained and applications discussed for each of these.

\section{8:00}

2aBB2. Measurement of oceanic bubble populations using broadband acoustics. Eric J. Terrill (Scripps Inst. of Oceanogr., Mail Code 0213, La Jolla, CA 92093-0213, et@mpl.ucsd.edu)

The size distribution of bubbles in the ocean will evolve with a number of different temporal and spatial scales as a result of both the physics which govern their formation (breaking wind waves, breaking waves in the surf zone, or ship wakes) and the physical processes which control their lifetimes: turbulent mixing, bubble rise speed, and gas dissolution. A broadband acoustic bubble sizing technique that is suitable for deployment in the rigors of the ocean environment has been developed and deployed on a number of occasions to study the physics of the bubble field and their implications to underwater sound propagation (Terrill and Melville, 2000). 
The technique is based upon inversions of direct acoustic attenuation and sound-speed measurements at frequencies ranging from approximately 2-200 kHz using broadband pulse transmissions at ping rates up to $25 \mathrm{~Hz}$ across short path lengths of $\mathrm{O}(0.1-1) \mathrm{m}$. The accuracy of the measured bubble size distributions has been confirmed with both internal consistency checks based on the complex dispersion relationship as well as laboratory comparisons with independent optical techniques. A review of the development of the bubble sizing instrumentation will be presented and results from a number of different field deployments discussed.

$8: 25$

2aBB3. Bubble characterization in the surf zone. Grant Deane (Scripps Inst. of Oceanogr., UCSD, La Jolla, CA 92093-0238)

Breaking waves entrain bubbles ranging in radius from tens of microns to centimeters. In the surf zone, these bubbles are evident above the ocean's surface as patches of foam that persist for a few seconds after the passage of a breaking wave. Beneath the surface, the bubbles are organized into dense plumes with void fractions of air exceeding 0.1 . In the absence of additional breaking, the void fraction of air decreases to 1e- 6 or less in a minute or so as bubbles rise to the surface, dissolve, and disperse. Thus, a full characterization of bubbles in the surf zone requires instruments that operate over a wide range of void fractions and bubble radii. In practice, a variety of techniques is used, and the application and results from a number of optical and acoustical instruments, including photographic techniques, travel time sonars, Doppler sonars, and acoustical resonators will be discussed. Some of the physical acoustical effects associated with the large populations of bubbles entrained by breaking surf, such as acoustic hot spots in surf noise and acoustic transmission dropouts, will also be presented.

8:50

2aBB4. On bubble-mediated scattering from the sea surface: Modeling, and field and laboratory measurements. Peter $\mathrm{H}$. Dahl (Appl. Phys. Lab., Univ. of Washington, Seattle, WA 98105)

Bubbles, located just below the air-sea interface, contribute significantly to the observed level of acoustic scattering originating from the sea surface, in contrast, for example, to microwave scattering. Typically, such bubbles are the result of breaking windgenerated waves, and thus their concentration is closely linked to wind speed. In this paper we discuss scattering from bubbles located within the proximity of the sea surface, with emphasis on the effects of the nearby reflecting surface, which gives rise to multiple paths from source to bubble to receiver. The effects of scattering geometry, e.g., as in bistatic forward scattering and monostatic backscattering, are illustrated using field data taken at a frequency of $30 \mathrm{kHz}$. An interpretive model for the contribution of near-surface bubbles to the apparent scattering cross section per unit area of sea surface is also discussed. This model has both a monostatic and a bistatic form, and, somewhat paradoxically, the bistatic form does not reduce to the monostatic form in the limit of source and receiver co-location. The issue is clarified, however, by examining the constituent pressures associated with the multiple paths, and by well-controlled laboratory measurements that include rough surface effects. These confirm both monostatic and bistatic forms of the model.

9:15

2aBB5. Acoustic cavitation and sonoluminescence. Thomas J. Matula (Appl. Phys. Lab, Univ. of Washington, 1013 NE 40th St., Seattle, WA 98105)

The violent collapse of a bubble in an acoustic field can lead to the emission of light, a phenomenon called sonoluminescence (SL). The study of sonoluminescence has led to refinements in understanding cavitation. This talk will focus on three areas of research in which the application of sonoluminescence is used to investigate cavitation: (1) In the field of single-bubble SL, techniques have been improved to study the dynamical motion of the bubble and, with comparisons to SL, models of the bubble dynamics have been refined. In particular, thermal conduction and the trapping of water vapor during the bubble collapse contribute significantly to the internal temperatures and to the dynamics of the cavitation bubble. (2) In lithotripsy, intense shock waves generate cavitation fields which play a role in kidney stone communition. In vitro studies have shown that SL can be correlated with a bubble's expansion and collapse within the field. (3) In sonochemistry, high-intensity ultrasound is used to initiate or enhance chemical reactions. SL is often observed under these conditions, and in some cases, there is a correspondence between SL intensity and sonochemical yield. Although often overlooked, it can be important to account for the physics and chemistry at the bubble surface.

\section{9:40-9:55 Break}

\section{9:55}

2aBB6. Microcavitation-assisted selective surface erosion. Sameer I. Madanshetty (Mech. and Nuclear Eng., Kansas State Univ., 322 Rathbone Hall, Manhattan, KS 66506)

Cavitation is a chance-dominated process and cavitation activity cannot be readily controlled. Interestingly, our recent research in ACIM (acoustic coaxing induced microcavitation) allows us to bring about microcavitation quite readily at all surfaces of interest. Liquid-borne microparticles exposed to near threshold strength sound fields do not ordinarily cause cavitation, however, experience with ACIM indicates that cavitation by the microparticles is readily facilitated and one can bring about controlled erosion of painted and inked surfaces. Cavitation is an energy concentrator and microcavitation implosions concentrate enormous energy at points. ACIM, consequently, can generate high-energy density points on liquid-borne surfaces. Since the implosion effects are felt only locally, these high-energy density points easily remove ink/paint off surfaces while preserving the structural integrity of the underlying 
substrate. The presentation will demonstrate initial evidence of using silent sound and clean water-controlled acoustic microcavitation for removing xerographic ink from paper, and paint layers from metal surfaces without damaging the underlying substrates, paper and metal, respectively. More significantly such microcavitation-assisted surface erosion permits an easy method for determining the bonding strength between the thin film and its substrate. [Work supported by Navy and NSF.]

\section{Contributed Papers}

\section{0:20}

2aBB7. Surf-zone bubble detection using multiple techniques: The Worbarrow Bay experiment. Timothy G. Leighton, Matthew D. Simpson, Steve D. Meers, Paul R. White (Inst. of Sound and Vib. Res., Univ. of Southampton, Highfield, Southampton SO17 1BJ, UK), Gary J. Heald, Hugh A. Dumbrell, James W. Clarke (DERA Bincleaves, Weymouth, Dorset, UK), Peter R. Birkin, and Yvonne Watson (Univ. of Southampton, Highfield, Southampton SO17 1BJ, UK)

This paper describes a multisensor experiment to characterize the bubble population and its effects in the surf zone. Active bubble detectors (combination-frequency sensors, and the inversion of sound speed and attenuation) provided estimations of the bubble-size distribution, and allowed better interpretation of more novel sensors. These include acoustoelectrochemical sensors, and estimations of the bubble population from the ring-up time associated with the insonification of the population, and from the ambient noise (attention being paid to how the ringing population so determined differs from the full population measured by active techniques). [Work supported by the U.K. Defence Evaluation Research Agency and the EPSRC.]

\section{0:35}

2aBB8. The effects of oceanic surfactants on acoustic propagation in bubbly liquids. Joseph C. Jankovsky and Ronald A. Roy (Dept. of Aerosp. and Mech. Eng., Boston Univ., 110 Cummington St., Boston, MA 02215, jankov@bu.edu)

The chemical composition of the top layer of the ocean is known to contain surface-active substances that are readily adsorbed to an air-water interface. The presence of surfactants in a liquid induces a viscoelastic stress along a two-dimensional interface. These surfactants can coat bubbles that alter their individual dynamics. A model is presented that incorporates the effects of surface viscoelasticity for acoustic propagation in bubbly fluids. The effects of both surface dilatational viscosity and surface elasticity on the phase speed and attenuation are considered. Surface viscosity is found to decrease the attenuation near bubble resonance frequencies, yet increase damping below resonance. Surface viscosity also diminishes the resonant effects for the phase speed in bubbly fluids. Both effects become significant for bubble sizes below 100 microns. The addition of surface elasticity is found to decrease the mean oscillation bubble radius, and thus shift bubble resonance to a higher frequency. The effects of the model on in situ acoustic bubble sizing methods will also be discussed. [Work supported by ONR.]

\section{0:50}

2aBB9. Acoustic scattering from an elastic tube filled with bubbly fluid. Preston S. Wilson, Ronald A. Roy, and William M. Carey (Dept. of Aerosp. and Mech. Eng., Boston Univ., Boston, MA 02215)

A complete model describing broadband sea surface scattering at high wind speeds has not been developed. One difficulty is accounting for scattering from near-surface bubble clouds. This problem has been addressed in the literature for low frequencies. To first order, an acoustically compact bubble cloud can be modeled as a compressible sphere, where the scattering strength depends only on spherical cloud volume and mean void fraction, not the bubble size distribution or cloud shape. This hypothesis has been experimentally tested using freely rising artificial bubble clouds [J. Acoust. Soc. Am. 92, 2993-2996 (1992)]. The measured lowfrequency monopole target strength of the cloud agreed with theory but higher-frequency results did not. To further understand scattering from these objects, laboratory scattering experiments are underway using geometrically well-characterized bubbly fluid targets. Initial measurements of scattering from a bubbly fluid-filled latex tube are presented and compared to an effective medium theory. These initial results lack independent void fraction determination but good qualitative agreement is found, even above the monopole resonance frequency. A new method used in these experiments to generate large volumes of nearly monodisperse bubbly fluid samples will also be described. [Work supported by ONR.]

\section{1:05}

2aBB10. Acoustic scattering from partially voided compliant and fluid spheres. Joseph C. Jankovsky, Ryan D. McCormick, Ronald A. Roy, and William M. Carey (Dept. of Aerosp. and Mech. Eng., Boston Univ., 110 Cummington St., Boston, MA 02215, jankov@bu.edu)

The presence of bubbles has been shown to change the compressibility and complex sound speed in a liquid. In the ocean, acoustically compact bubbly mixtures manifest themselves as highly compressible regions that effectively scatter low-frequency sound. To study low-frequency sound scattering, multifrequency backscattering experiments have been performed in a tank using partially voided three-quarter-inch diameter polyurethane spheres as targets. Target strengths $(2-20 \mathrm{kHz})$ were measured for four spheres with void fractions of $0 \%, 3.4 \%, 4.2 \%$, and $6 \%$. Measured target strengths for the voided spheres were on the order of -40 to -60 $\mathrm{dB}$ (re $1 \mathrm{~m}$ ). The frequency response exhibited modal structure, with peaks shifting to lower frequencies for higher void fractions. No backscatter signal was detected for the solid polyurethane sphere. Target strength was also measured for a hollow polyurethane sphere containing a suspension of bubbles in polymer gel. The void fraction was determined by fitting the scattering theory and low-frequency bubbly fluid compressibility model to the measured data. [Work supported by ONR.]

\section{1:20}

2aBB11. Comparing the predictions of a numerical model of SBSL in a variable acceleration environment to experiment. Charles Thomas, Sean Wyatt, ${ }^{\text {a) }}$ Ronald Roy, and R. Glynn Holt (Dept. of Aerosp. and Mech. Eng., Boston Univ., 110 Cummington St., Boston, MA 02215)

The results of an August 1999 KC-135 SL experiment will be discussed and compared to a numerical model [Wyatt et al., J. Acoust. Soc. Am. 106, No. 4, Pt. 2, 2290 (1999)] for SL in a cubic acoustic resonator in a varying acceleration environment. The model takes into account gravitational effects and varying ambient pressure effects, and predicts the maximum bubble size, light intensity, and levitation position of the bubble. A review of and comparison to other investigators experimental results will be included. [Work supported by NASA.] ${ }^{\text {a) }}$ Currently at Ford Motor Company, Detroit, MI.

\section{1:35}

2aBB12. Sonoluminescence at the nanoscale. Carlos G. Camara, Keith R. Weninger, and Seth J. Putterman (Phys. Dept., Univ. of California, Los Angeles, Los Angeles, CA 90095)

Sonoluminescence (SL) has been observed to be robust over a wide parameter space ranging from $8 \mathrm{kHz}$ to $11 \mathrm{MHz}$. Although some lines can be discerned in the spectra of some cavitation clouds, they sit on top of a broadband ultraviolet continuum that spans to at least $6 \mathrm{eV}$. Experiments and theory indicate that the light-emitting region can reach tenths of nanometers. Although the SL mechanism and its huge parameter space remain a mystery, it has already been put to use as a surgical device. At 30 $\mathrm{kHz}$ it is used for internal lipectomy and at $1 \mathrm{MHz}$ it is used for externally assisted lipectomy. At $11 \mathrm{MHz}$ a dense cloud of light-emitting bubbles is observed in the far field of the transducer. These observations could be exploited for future uses of nonintrusive ultrasound surgery. 
conventional chemical reactions, the intensity of luminescence was expected to increase with stirring operation. At 28 and $100 \mathrm{kHz}$, the lumi-

2aBB13. Anomalous changes of sonochemical luminescence intensity under stirring operation. Hideto Mitome (Natl. Industrial Res. Inst. of Nagoya, AIST, Nagoya, 462-8510 Japan, mitome@nirin.go.jp) and Shinichi Hatanaka (Japan Sci. and Tech. Corp., Kawaguchi, Saitama, 332-0012 Japan)

Measurement of sonoluminescence is an effective method to evaluate a reaction field induced by intense ultrasound in a liquid. The present paper discusses changes of intensity of sonochemical luminescence from a luminol solution under various stirring conditions. A glass beaker filled with a luminol solution was irradiated by ultrasound using Langevin-type transducers attached to the bottom and side walls of a cooling bath. As in nescence intensity increased at a higher applied power and at a faster stirring speed. But, at $45 \mathrm{kHz}$ where intense cavitation was observed in the present apparatus, the luminescence intensity decreased as the applied power increased while stirring with a flat paddle. This anomalous behavior was more pronounced at more intense stirring operation with an impeller. The authors reported the existence of upper threshold of sound pressure for sonoluminescence [Hatanaka et al., Jpn. J. Appl. Phys. 38, 3053-3057 (1999)]. The present results suggest that stirring operation affects the generation and growth of cavitation bubbles making them exceed the upper threshold in some cases, and that there is an optimum stirring motion for sonochemical reactions.

\title{
Session 2aEA
}

\section{Engineering Acoustics: Ultrasonic Sensors and Motors}

\author{
Harold C. Robinson, Chair \\ Naval Surface Warfare Center, Code 2131, 1176 Howell Street, Newport, Rhode Island 02841-1708
}

Chair's Introduction-8:35

Invited Papers

8:40

2aEA1. Ultrasonic motors (USM) - An emerging actuation technology for planetary applications. Yoseph Bar-Cohen, Xiaoqi Bao, and Hari Das (JPL/Caltech, MC 82-105, 4800 Oak Grove Dr., Pasadena, CA 91109-8099, yosi@jpl.nasa.gov)

Efficient miniature actuators that are light, compact, and driven by low power are needed to drive telerobotic devices and space mechanisms in future NASA missions. Examples of space mechanisms and devices that require actuators include robotic arms, miniature rovers, release mechanisms, positioning devices, aperture opening and closing devices, and real-time compensation for thermal expansion in space structures. These motors need to operate at various temperatures and pressures with a large range of thermal variations over a relatively short period swing. Ultrasonic rotary motors have the potential to meet this NASA need and they were developed as actuators for miniature telerobotic applications. These motors were adapted for operation in the environment of Mars, which includes very low temperatures and vacuum. A hybrid analytical model, including the influence of the rotor and stator dynamics, friction effects, and interface effects, was developed to design an efficient ultrasonic motor as a complete system. In parallel, efforts have been made to determine the thermal and vacuum performance of these motors, and effective operation at temperatures as low as $-150{ }^{\circ} \mathrm{C}$ and at a pressure of 16 mtorr were demonstrated. To explore telerobotic applications for USMs a robotic arm was constructed with such motors.

2aEA2. Bio-inspired acoustic sensors based on artificial stereocilia. Flavio Noca, Michael Hoenk, Brian Hunt (Jet Propulsion Lab., 4800 Oak Grove Dr., Pasadena, CA 91109, flavio.noca@jpl.nasa.gov), Petros Koumoutsakos, Jens Walther, and Thomas Werder (ETH Zurich, Zurich, Switzerland)

A unique, biologically inspired acoustic transducer based on artificial stereocilia is being developed. This transducer will enable directional sensitivity and miniaturization of acoustic sensors while enhancing sensitivity, ultimately leading to revolutionary advances in acoustic detection and signal processing. The similarities between natural stereocilia and the proposed transducer array could allow for the first time the fabrication of an artificial cochlea that relies on biologically inspired signal-processing techniques. Other applications, such as measuring sounds generated by moving micro-organisms and nanoscale biological events (metabolic flows), may be enabled by this novel transducer. Finally, artificial stereocilia (functioning as actuators instead of sensors, analogous to stridulatory pegs in insects) will be capable of generating acoustic signals for applications in active acoustic instruments such as sonar. Before these advantages can be realized, fundamental developments are required in the design and fabrication of a transducer based on artificial stereocilia arrays. The potentially revolutionary nature of this transducer is being demonstrated by fabricating and characterizing acoustic sensors based on recently produced carbon nanotube arrays. 
2aEA3. A surface acoustic wave hygrometer for high-resolution measurements of atmospheric humidity. Michael E. Hoenk, Greg Cardell, Flavio Noca, and Robert K. Watson (Jet Propulsion Lab., California Inst. of Technol., Pasadena, CA 91109)

Weather and climate depend on atmospheric phenomena at all scales, from large-scale atmospheric circulation to microphysical processes. In this talk, a surface acoustic wave (SAW) hygrometer developed at JPL for high-resolution measurements of atmospheric humidity will be discussed. Flight tests of an early prototype on the NASA DC8 showed more than an order of magnitude faster response than chilled-mirror hygrometers. In 1998, a 1-kg reference radiosonde based on the SAW hygrometer was flown on a small balloon to an altitude of 44000 feet, while a mobile ground station recorded telemetered data containing high-resolution measurements of humidity, pressure, temperature, and GPS position. Later in 1998, the SAW hygrometer was deployed on the NASA DC8 to measure humidity in Atlantic hurricanes. In 1999, the SAW hygrometer recorded humidity in the test flight of NASA's Helios unpiloted aircraft. Currently, this instrument is being modified for incorporation into a hand-held instrument for monitoring environmental humidity on the space station. [The research described in this paper was performed at the Jet Propulsion Laboratory, California Institute of Technology, and was jointly sponsored by the National Aeronautics and Space Administration, Earth Science Enterprise, Space Science Enterprise, and Human Exploration and Development of Space Enterprise.]

\section{9:55-10:10 Break}

\section{0:10}

2aEA4. The zNose, a new electronic nose using acoustic technology. Edward J. Staples (Electronic Sensor Technology, LP, 1077 Business Ctr. Circle, Newbury Park, CA, staples@estcal.com)

An array of sensors simulating the human olfactory response has become known as an electronic nose, or eNose. An eNose provides a vectorial image in $N$-dimensional space (where $N$ equals the number of sensors) of specific vapor mixtures containing possibly hundreds of different chemical species. Typically, eNoses have only a few sensors, produce responses that are not correlated, and have poor sensitivity. A new type of electronic nose, based upon fast chromatography and a single high-Q acoustic sensor, solves these problems by stimulating a virtual sensor array containing hundreds of orthogonal sensors. Analysis of any odor is accomplished by serially polling this virtual sensor array or a spectrum of retention times. This paper will describe the acoustic technology as well as present results for applications involving important vapors associated with several commercial foods and beverages. Part per billion sensitivity has been achieved with volatile compounds and part per trillion sensitivity for semi-volatile compounds. Because the new acoustic technology is quantitative it is the only electronic nose technology to be validated by the US EPA. The commercial expression of this technology, the zNose, is now providing an on-line quantitative measure of quality for food, beverages, cosmetics, and other aromatic products.

\section{$10: 35$}

2aEA5. Recent developments in transducers: Opportunities for the future. Ilene Busch-Vishniac (Whiting School of Eng., Johns Hopkins Univ., NEB 120, 3400 N. Charles St., Baltimore, MD 21218)

Most innovations in sensors and actuators result either from the introduction of new materials, or the exploitation of newly discovered mechanisms for conversion of energy between the electrical and mechanical domains. This talk reviews new recent transducer improvements and suggests opportunities for the future of ultrasonic sensors and actuators. Although many of the transducer examples portrayed were not conceived for ultrasonic or acoustic applications, the exploitation of their materials and energy conversion mechanisms could provide a means of enhancing the arsenal of ultrasonic transducers available.

\section{Contributed Paper}

\section{1:00}

2aEA6. MEMS condenser microphone. Peter J. Henning and Torben Storgaard-Larsen (Bruel \& Kjaer, 2850 Naerum, Denmark)

The measurement microphones of today are the results of almost 50 years of continuous optimization of production methods based on precision mechanics. Scientists have searched for new ways of measuring sound, which can reach the performance of the condenser microphone, but so far have not succeeded! However, applying new materials and production methods, the window of achievable specifications of condenser mi- crophones can be widened. Combining the accuracy and reliability of silicon micro machining with the experience gained on traditional measurement microphones, unique new features can be added to the range of microphones. As a first result of such efforts, a new microphone unit, the multifield microphone, has been developed. The multifield microphone has a "flat" frequency response in all sound fields, i.e., pressure field, diffuse field, or free field (when the sound comes from different angles of incidence). This results in higher accuracy in most measurement situations, where the sound field is nonidealized. 


\title{
Session 2aNSa
}

\section{Noise and NOISE-CON: Soundscapes}

\author{
Brigitte Schulte-Fortkamp, Cochair \\ Department of Physics/Acoustics, Oldenburg University, Oldenburg D-26111, Germany \\ Joseph Pope, Cochair \\ Pope Engineering Company, P.O. Box 590236, Newton Centre, Massachusetts 02459-0002
}

Chair's Introduction-8:40

Invited Papers

8:45

2aNSa1. Can soundscapes work as a moderator concerning noise annoyance? Brigitte Schulte-Fortkamp (Dept. of Phys./ Acoust., Oldenburg Univ., 26111 Oldenburg, Germany, brigitte@aku.physik.uni-oldenburg.de)

The evaluation procedure to detect and to analyze soundscapes in their meaning regarding noise annoyance measurement is a new step and a very complex procedure. Focusing on the interaction of people and noise, different aspects like the structure of urban areas, people living in those areas, architectural and social parameters designing those areas, and acoustical and visual parameters will be taken into account for analyses. Moreover socio-cultural- and lifestyle-related elements of soundscapes have to be discussed concerning the aspects of annoyance measurement which usually are focused on interference, disturbance, sources of annoyance, and predicting noise annoyance. Basically, in the discussion of both procedures it is questioned whether soundscapes can work as a moderator concerning annoyance. Detailed aspects may lead to the question of correlations between annoyance judgments and somatic processes, and what happens when people give an overall judgment on the degree of annoyance. Future research is needed to explore soundscapes moderating the judgment on life situation and noise sources.

9:05

2aNSa2. Soundscapes in American mainstream films. Barbara Flueckiger (Inst. of Film Theory, Univ. of Zurich, Plattenstrasse 54, CH-8032 Zurich, Switzerland, zauberklang@bluewin.ch)

The question of how films establish an acoustic environment was studied in the course of a research project on sound design in American mainstream film. The study was supported by the Swiss National Foundation. The corpus of this research project consisted of 96 films, their production years ranging from 1926 to 1995. Most of these films received an Academy Award for Best Sound. They were closely examined regarding their strategies to establish fictional, yet natural-seeming, soundscapes. It was discovered that the film industry developed a rather restricted vocabulary to display certain types of geographically, socially, and/or culturally defined places. The cause of the restriction can be found in technical and historical reasons as well as psychological considerations. Film soundscapes have a clear communicative function in contrary to natural soundscapes, which contain random noises. According to an unwritten Hollywood rule of the so-called classic era of the thirties and forties, any distraction from the narrative goal had to be avoided. This rule, however, was revised when multichannel formats were developed, first in the fifties with different wide-screen systems, and later in the mid-seventies with the Dolby stereo 4- to 6-channel sound system.

9:25

2aNSa3. Sound quality of exterior vehicle noise. Klaus Genuit (HEAD Acoustics GmbH, Eberstrasse 30a, 52134 Herzogenrath, Germany)

For several years the acoustical engineers from all car manufacturers have been busy investigating and improving the sound quality of the interior noise of vehicles. For 20 years we have been using specific measurement technologies and analyses to describe sound quality with consideration of the signal processing of human hearing. But with respect to the exterior vehicle noise only standard measurements, e.g., the A-weighted sound pressure level, are still in use. Although the A-weighted sound pressure level has been reduced, the annoying effect of exterior vehicle noise and traffic noise has increased. The negative impact of exterior vehicle noise and traffic noise on man must be reduced. Solutions must therefore be found to the vehicle noise emission problem while allowing sustainable mobility. An important prerequisite for improvement of the well-being of the public and acceptance of future traffic and acoustic environments is the consideration of human sound perception of the exterior vehicle. This paper describes how we can transfer the knowledge of sound quality analysis with respect to interior noise to the evaluation of exterior vehicle noise. This includes not only the methodologies for the measurement and analyses, but also the development of a prediction tool. 
2aNSa4. Soundscapegraphy of old-town of Kyoto with the Gion Festival. Kozo Hiramatsu (Mukogawa Women's Univ., 663-8558 Nishinomiya, Japan, NBG01036@nifty.ne.jp)

The soundscape of the Yamahoko-cho area in the old capital of Japan, Kyoto, is described from various aspects based on an intensive survey of acoustic ecology. The area located in the city center is famous for the Gion Festival, which is one of the biggest and oldest festivals in the country. The area's soundscape on ordinary days is basically dominated by the noises of automobiles passing through the streets, since there are also companies and shops in the area, which is the trading center of Japanese costumes, kimono. In July, when the ceremony and festival are undertaken for 1 month, the soundscape changes to be dominated by a variety of sounds related to the festival. Particularly during the days when bands of music by flutes, drums, and small gongs are performed, the soundscape of the area becomes a complete festival one. The music is so famous that it is recognized as characteristic of the city. The residents in the area are raised listening to the sounds in summer, and these sounds are closely connected with their lives. Though the music is no doubt the loudest sound heard in the area, no one complains.

\section{0:05}

2aNSa5. Factors moderating the effect of noise barriers. Tommaso Meloni and Fredy Fischer (Swiss Agency for the Environment, Forests and Landscape, 3003 Berne, Switzerland, tommaso.meloni@buwal.admin.ch)

More often than not, on noise research the dose-response relationship shows a modest explained variance. A field study was carried out to investigate the effects of 13 different noise barriers, and 500 people exposed to noise were surveyed using questionnaires. First it seems that the acoustic effectiveness of the noise barriers is responsible for the reduction in annoyance. However, a detailed analysis shows that further factors moderate the relation between the noise parameters and the reaction to noise. It seems that simply introducing a noise barrier can cause a reduction in annoyance to an extent equivalent to $5 \mathrm{~dB}$. This confirms the fact that the outdoor noise levels and the acoustic design of the surroundings are among the principle parameters influencing annoyance. In addition, annoyance is determined by individual acceptance of noise barriers. The degree of acceptance splits the reaction curve with a separation around 4 units on the rating-scale of 11 units of annoyance. The design of the noise barrier partly affects this splitting. There is a higher degree of acceptance of naturally constructed and transparent noise barriers than for ones that are specially formed. Other determining factors for annoyance will be discussed.

\section{0:25-10:45 Break}

\section{Contributed Papers}

\section{0:45}

2aNSa6. Quiet areas and quiet facades: Important elements in the EU Noise policy. Tor Kihlman (Dept. of Appl. Acoust., Chalmers Univ. of Technol., SE 41296 Goteborg, Sweden)

The long-term goal for road traffic noise, Lden $<55 \mathrm{~dB}$, cannot be reached for all dwellings either in sprawled or in compact cities during the foreseeable future [T. Kihlman and W. Kropp, "Limits to the Noise Limits," ICA, Seattle 1998]. What, then, can be done to improve the situation? One strategy is to exploit the spatial noise level variations. Even though many dwellings are exposed to Lden $=65-70 \mathrm{~dB}$, outdoor levels in the range 40-50 Lden are not rare in the cities: courtyards in European cities being the typical case. The EU draft directive on ambient noise puts emphasis on the protection of quietness. Quiet areas and "quiet facades" shall be shown on noise maps. The concept of a relatively quiet facade has been introduced. Questions to answer are: What are the effects on the annoyance and sleep disturbance when dwellings have one noisy and one quiet side? To what extent do existing, noise-exposed dwellings also have a quiet side? What are the potentials to increase also at low cost-the access to quietness in the urban renewal process? Some results from ongoing research will be presented as well as plans for further research.

\section{1:00}

2aNSa7. Measurement of the natural soundscape in national parks. J. Micah Downing (Wyle Labs., 2001 Jefferson Davis Hwy., Ste. 701, Arlington, VA 22202, mdowning@arl.wylelabs.com) and Eric Stusnick (San Jose, CA 95135)

The National Park Service (NPS) has identified the natural soundscape in its various park units as one of the resources it has been mandated to protect. Natural soundscape is defined as the acoustic environment that would exist in the absence of human-related activity. Accordingly, the
NPS has developed policies related to soundscape management, preservation, and restoration, which require information about the natural ambient sound levels in all of their properties throughout the country. As part of this process, metrics and procedures for measuring the natural soundscape and assessing non-natural intrusions have been developed. Two general types of procedures have been developed: one based on audibility considerations, the other based on acoustic energy measurements. This paper describes the work that has been carried out in developing and assessing these procedures.

\section{1:15}

2aNSa8. Measurement and modeling of snowmobile noise and audibility at Yellowstone and Grand Teton National Parks. Christopher Menge and Jason Ross (Harris Miller Miller and Hanson, Inc., 15 New England Executive Park, Burlington, MA 01803)

The U.S. National Park Service is concerned about the effect that noise from snowmobiles and other over-snow vehicles has on the natural soundscapes in the National Parks. This paper addresses the measurements and modeling performed to assess both the total area in the parks where snowmobile noise would be audible and the noise intrusiveness as a function of distance from the trails. The work was performed in support of an Environmental Impact Statement that evaluated seven operational transportation alternatives in the two parks. The modeling approach computed both A-weighted sound levels and audibility continuous time histories of snowvehicle noise at several distances from the trails. Acoustical input to the model included $\frac{1}{3}$-oct band levels for ambient conditions, vehicle source levels, and propagation of sound over a snow-covered surface. Consequently, distances to the onset of audibility detection, areas of the parks affected, and statistics on received sound levels were calculated. The paper presents both the modeling approach and examples of results. 
2aNSa9. The application of a noise-rating scale composed of hue-scale and $L_{\text {Aeq }}$ on various kinds of noise sources for noise map. Kenji Furihata and Takesaburo Yanagisawa (Dept. of Elec. and Electron. Eng., Faculty of Eng., Shinshu Univ., 4-17-1 Wakasato, Nagano, 380-8553 Japan, kennfur@gipwc.shinshu-u.ac.jp)

In addition to reporting the results of measurements of existing environmental noise and the results of calculations of noise from projected activities, the application of color for representing in terms of noise zones may be useful (ISO 1996-2). Between color and noise, in spite of differences, there exists an interaction, although the connection between the visible and the audible expression is subjective. Subjectivity can in a given moment become objective, if one succeeds in unifying the visible and the audible. In the case of colored paper on a white background, the relation between the hue level expressing the psychological effect (annoyance) and $L_{\text {Aeq }(5 \min )}(\mathrm{dB})$ of typical noise sources was discussed in a laboratory experiment. According to the obtained noise-rating scale, the seven-hue scale and $L_{\text {Aeq }(5 \mathrm{~min})}$ are correlated well $(r=0.874)$ as follows: white (value V9; not at all bothersome) $\Leftrightarrow$ below $26 \mathrm{~dB}$, light blue (hue $2.5 \mathrm{~B}$, value $8 /$ chrome 4 ; not bothersome) $\Leftrightarrow 26$ to $37 \mathrm{~dB}$, light green (2.5BG, 7/8; not too bothersome) $\Leftrightarrow 37$ to $49 \mathrm{~dB}$, bright yellow (7.5YR, 7/14; a little annoying) $\Leftrightarrow 49$ to $61 \mathrm{~dB}$, brown $(10 \mathrm{R}, 4 / 8$; annoying) $\Leftrightarrow 61$ to $70 \mathrm{~dB}$, purple $(2.5 \mathrm{P}, 4 / 10$; very annoying $) \Leftrightarrow 70$ to $80 \mathrm{~dB}$ and red $(5 \mathrm{R}, 5 / 14$; extremely annoying) $\Leftrightarrow$ over $80 \mathrm{~dB}$
2aNSa10. Methodology for analyzing perceptual criteria of urban soundscape. Raimbault Manon (CERMA UMR CNRS 1563 Rue Massenet, 44000 Nantes, France)

The exposed study is part of a larger program which intends to constitute an urban soundscape simulator. This paper presents analyses of urban scenes with the aim of identifying parameters of sound perception for this future model. It seems that the perception of everyday urban sounds is not yet well understood by urban planning actors. However, those considerations are more and more problematic in everyday life and are becoming increasingly important for town planner recommendations. Therefore the assessment of perception indicators linked to acoustical measures is intended to help one fully understand urban acoustics phenomena. The method consisted of two simultaneous procedures on site. In order to calculate acoustical parameters, we recorded samples of soundscape that we analyzed afterwards. At the same time we observed perceptual attitudes and opinions using a semantic differential inquiry of passersby. The locations were chosen in order to represent the main places of European city centers. Experimental results showed that five sector-related indicators could be defined. A full understanding of urban acoustical phenomena needs this multidisciplinary approach so that both physical and psychosociological parameters can be integrated in town planning.

Session 2aNSb

\title{
Noise and NOISE-CON: Power Plant Noise Control and Prediction and Industrial Noise
}

\author{
Frank H. Brittain, Chair \\ Bechtel Corporation, 50 Beale Street, San Francisco, California 94105
}

Chair's Introduction-7:55

Invited Papers

\begin{abstract}
8:00
2aNSb1. Noise control engineering challenges in designing power plants to meet noise limits. Robert Putnam (Siemens Westinghouse, 4400 Alafaya Trail, Orlando, FL 32826)

Noise control considerations play a vital and often pivotal role in a broad range of issues in the design of modern power plant facilities. The entire design process, from site selection, through final operational acceptance, is affected by the proper integration of the governing noise criteria, especially when relatively low levels are called for. The noise control engineer is continually challenged to justify added noise control measures, maintain adequate design margins, minimize costs, performance impacts, and design impacts, while coordinating a number of cross-discipline interfaces ranging from marketing and sales to contracts to startup and field engineering. This paper presents an overview of the principal noise control engineering tasks involved when effectively integrating required noise control features into the design process, highlights key interface requirements, and provides notes on the practicality, safety, and maintainability of control features. Illustrative examples from the author's experience, both positive and negative, will be included.
\end{abstract}

8:20

2aNSb2. Using a prediction model to allocate allowable noise between sources and establish equipment noise limits. David J. Parzych (Power Acoustics, Inc., 12472 Lake Underhill Rd. \#302, Orlando, FL 32828, dparzych@ poweracoustics.com)

Power generation facilities typically require customized noise abatement features to achieve compliance with various local and state noise regulations. Differing equipment or equipment arrangements, size and placement of equipment on the plant owner's property, and location within a community can all affect the amount of noise control necessary for a given facility. Selecting the correct amount of silencing for each piece of plant equipment is essential when optimizing for reduced cost of the overall plant noise control treatments. To achieve the overall plant noise goals, an analytical noise prediction model of the facility is built and exercised with various equipment options until the desired goal is achieved. The paper describes methods of using a noise prediction model to 
allocate noise "budgets" among sources, and using the model to establish noise criteria in specification form. The discussion will address the overall effects of starting with poorly defined data, as well as imprecise or erroneous noise source data supplied by equipment manufacturers. Estimating reasonable and achievable noise goals for the equipment will be addressed.

8:40

2aNSb3. Integrating noise controls into the power plant design. Wayne E. Bradley (Stone \& Webster Eng. Corp., 245 Summer St., Boston, MA 02210, wayne.bradley@ stoneweb.com)

The selection of noise controls and the specification of low-noise equipment is an important part of designing a power plant to meet specific noise limits. However, successfully interfacing with the design team and integrating these controls into the project design is an equally demanding task. The noise-control specialist must work closely with the design team to assure that the affects of the mitigation on space allocation, equipment performance, energy consumption, cooling, access to equipment, and safety are appropriately considered. Each of these factors must be addressed to assure that the controls work effectively under plant operating conditions. The design process is iterative-design details and controls may change as the design progresses. Both formal and informal lines of communication must be cultivated with the design team, and the mitigation recommendations made to them should be followed up to assure their proper incorporation into the project design. This paper describes these processes.

\section{9:00}

2aNSb4. Noise-control engineering interacting with the design process of a power plant. Marlund E. Hale (Parsons Eng. Sci., Inc., 100 W. Walnut St., Pasadena, CA 91124)

There are many challenges which face the noise-control engineer associated with developing and implementing both the occupational and community noise controls for power generation facilities. They include becoming an early and viable part of the proposal and design process and maintaining a proper and profitable business effort. Challenges during the proposal and design phase include working within the business plan, dealing with cost estimates incorporated during the development of the winning proposal budget, the project design schedule, the engineering process, and the noise-control cost estimating and procurement process. Once a project has been won, certain financial and scheduling commitments are already in place. The project design team is then assembled and the design evolves conceptually as the various issues affecting the project are incorporated. To be a viable and effective part of the design team, the noise-control engineer must become an early player, deal with an incomplete and evolving design, keep communication lines open, stay abreast of the many facets of the design evolution, communicate project-impacting information to project management, interface with the procurement process, and stay in a position to interface with and support engineering and construction inspectors.

2aNSb5. Risks in designing power plants to meet noise limits and how to manage them. Frank Brittain (Bechtel Corp., 50 Beale St., San Francisco, CA 94105)

Risks and risk avoidance affect both strategy and many choices made in designing power plants to meet noise limits. Each player typically attempts to avoid risk by passing off as many as feasible to other players. Many of these risks can readily be anticipated, but others are often unexpected. Each player-noise control engineer, engineer-constructor, owner, banker, and regulator-has somewhat different risks. Many factors can affect risks, including technical uncertainties, noise criteria, unreliable noise source data, regulatory environment, limits to the state-of-the-art, costs, schedule, any lack of experience of the responsible noise control engineer, and equipment that does not meet its noise limits. Risks are greatly increased when liquidated damages are present, the responsible noise control engineer is not adequately supported, and noise control is neglected or initiated too late in the design process. All of these factors are interrelated in complex ways. To successfully design a power plant, risks and risk avoidance should be understood, and strategies developed to reduce those risks. This paper builds on previous papers in the Special Session, reviews the risks of each player, and suggests strategies to reduce risks. Further, the ways in which risk affect the design process are identified.

\section{9:40}

2aNSb6. Strategy for writing procurement specifications. George F. Hessler, Jr. (Hessler Assoc., Inc., 3862 Clifton Manor Pl., Haymarket, VA 20169, gfhhai@msn.com)

The key word for writing workable procurement specifications is clarity. The recipient is much more interested in responding when the specification requirements are understood and unambiguous. As a minimum, the procurement specification must describe the starting point and end result specified by the author. The method of test verification and allowable tolerances, if any, is critical and must be defined in the procurement specification. Current acoustical requirements for power plant equipment must be specified in the near and far field with a clear order of precedence if there is a conflict between the two locations. The trend today for specifying far-field sound levels is to limit the specified parameter to the overall "A"'-weighted sound level. Since this parameter offers no chance of preventing excessive low-frequency noise, the " $\mathrm{C}$ "-weighted limit, as a minimum, must also be concurrently specified for all types of power plants.

\section{0:00}

2aNSb7. Customer sound criteria and vendor acoustical performance guarantees: The difficulties of meeting the first and verifying the second. Robert S. Johnson, Sr. (Solar Turbines, Inc., 9280 Sky Park Court, San Diego, CA 92131)

In their procurement specifications, gas turbine end users include acoustical criteria. Gas turbine manufacturers, in accepting the criteria, specify the performance of acoustical components which are procured from acoustical component vendors for installation on the sound sources supplied by the gas turbine manufacturer. If site noise problems develop, the sound sources must be investigated 
and the performances of the acoustical components evaluated to determine if the performance guarantees have been met. This paper discusses one gas turbine manufacturer's experience with how sound criteria are specified and responded to. Also discussed, are problems associated with the verification of vendor product performance guarantees, and the resolution of perceived product performance deficiencies.

10:20-10:40 Break

\section{Contributed Papers}

\section{0:40}

2aNSb8. Active control of low-frequency turbine exhaust noise. Bruce Walker, Alan Hersh, Joseph Celano (Hersh Walker Acoust., 780 Lakefield Rd., Unit G, Westlake Village, CA 91361), and Eldon Ray (Braden Manufacturing, Tulsa, OK 74101)

Gas turbine generators radiate noise from their exhaust stacks that contains substantial acoustical energy at very low frequencies. This kind of noise can result in community annoyance due to window rattling and other structural vibration excitation as well as disturb residents. Because of the long wavelengths of sound at these frequencies, typical duct sound absorbing treatments are inefficient, large and expensive. A research program was conducted to demonstrate the ability of a Helmholtz-resonatorbased active noise control system to attenuate low-frequency noise in a heated exhaust stack. A unique thermal stratification concept was developed that successfully protected the sound sources from hot exhaust flue gases. The thermal stratification concept permitted the use of both conventional off-the-shelf JBL 18-in. electrodynamic actuators and customfabricated piezoceramic sound sources in the demonstration program. Test results showed that $14-22 \mathrm{~dB}$ of noise attenuation was achieved over the frequency range $20-32 \mathrm{~Hz}$ at flue temperatures between $80^{\circ}$ and $1300^{\circ} \mathrm{F}$.

\section{0:55}

2aNSb9. Certifying noise emissions from heat recovery steam generators (HRSG) in complex power plant environments. George F. Hessler, Jr. (Hessler Assoc., Inc., 3862 Clifton Manor Pl., Haymarket, VA20169, gfhhai@msn.com)

HRSG units are used to generate steam by capture of heat from the combustion turbine exhaust gas, typically at $1000^{\circ} \mathrm{F}$. Combustion turbines, nominally in the 100-200-MW size, generate approximately 150 $\mathrm{dB}$, re $1 \mathrm{pW}$ " $\mathrm{A}$ " weighted sound power. Hence, noise emissions from the HRSG wall surfaces and stack outlet are a significant source of power plant noise in today's combustion turbine-based plants. Noise mitigation measures are required for most installations, and it is the responsibility of the HRSG supplier to engineer, furnish, and warrant the performance of the HRSG assembly. Verification of HRSG noise emissions in the field is difficult since the units are typically surrounded by countless other plant noise sources in complex arrangements. There is no applicable test standard in place for reference. This paper presents alternative methods of isolating and verifying HRSG noise source emissions in complex environments, and recommends test methods based on experience.

\section{1:10}

2aNSb10. Determination of sound pressure levels in situ using sound intensity measurements. Stephen E. Keith, Vincent Chiu (Health Canada, Radiation Protection Bureau, 775 Brookfield Rd., 6301B, Ottawa, ON K1A 1C1, Canada, skeith@hpb.hwc.ca), and G. Krishnappa (Natl. Res. Council of Canada, Ottawa, ON K1A 0R6, Canada)

Measurements of emission sound pressure levels of machinery require either specially defined test rooms or calculated corrections for the acoustic environment. In principle, it is possible to determine emission sound pressure levels from sound intensity measurements at specified work stations in any test environment if the requirements of background noise levels and field indicators are fulfilled. The draft international standard ISO $11205 / C D$ specifies such a method. In this paper the accuracy of emission sound pressure levels using sound intensity measurements was examined for three small sources in three acoustic environments, an ane- choic environment with loudspeakers to simulate background noise, an office environment, and a reverberant environment inside a stairwell. In the first two environments good measurement accuracies, within $1 \mathrm{~dB}$, were obtained. Sound intensity measurements by pointing the probe towards the source were as accurate, and simpler than computation of the resultant intensity using three arbitrary orthogonal measurements. As predicted by field indicators, measurements in the stairwell gave unacceptable errors for all three sources.

\section{1:25}

2aNSb11. Diagnostics of vibration excitation mechanisms for complex hydromechanical systems. Andrei B. Prokofiev, Valeri V. Lenchine, and Evgeniy V. Shakhmatov (Inst. of Acoust. of Machines, Samara St. Aerosp. Univ., Moskovskoe shosse 34, Samara 443086, Russia)

It is known that high-amplitude vibration can significantly impair reliability of technical tools. Sometimes it is difficult to identify the mechanism of vibration excitation in complex hydromechanical systems with many sources of oscillation. Simple frequency and correlation analysis does not provide all needed information. It is suggested to perform both usual vibration analysis in frequency domain and check out fluid-induced vibrations and conditions for auto-excitation caused by interaction of reciprocating mechanical parts and pulsing fluid. A case with an ammoniac compressor station is considered as an implication of the method. During the compressor station exploitation vibration has been observed with a frequency of $18.8 \mathrm{~Hz}$ that propagates through the system and exceeds the permitted level by 2.23 times. The frequency does not correspond to any known sources of oscillation for the system. The analysis showed that the back valve and attached pipes represent an auto oscillation contour that generates both low-frequency pulsation of fluid and mechanical vibration. It represented results of operational development of the compressor station, using the proposed technique that allowed the reduction of essential vibration of the system and generated noise.

\section{1:40}

2aNSb12. The mufflers for exhaust valves of industrial equipment. Valeri V. Lenchine (Inst. of Acoust. of Machines, Samara St. Aerosp. Univ., Moskovskoe shosse 34, Samara 443086, Russia)

The operating of exhaust valves of pneumatic cylinders and other pneumo-automatics causes impulse noise generation. Staff, working on half-automated conveyers and package lines, are often exposed to the high-intensity impulse noise, which is a reason for hearing loss. The design of a silencer for steady flow is well explored but there is not as much attention for an impulse noise attenuator. Moreover, mufflers for discharge valves of industrial equipment must meet additional requirements because an air exhaust emits broadband sound and an implication of mufflers with essential aerodynamic drag results in an operational disorder for pneumoautomatics. The long lifetime and simple maintenance are also significant for a consumer. The muffler's model takes into account an attenuation performance, calculation of a silencer pressure drop, and prediction of mass and size of the device that allows us to work out multi-criteria optimization tasks. There are considered peculiarities of impulse noise muffler design for the packaging pneumatic automates. The mufflers have low aerodynamic drag, are easy to install, inexpensive, and reliable. They provide the best acoustical performance for frequencies 500-4000 Hz. The comparison of effectiveness is represented for the different silencers. There are given examples of successful implementation of the mufflers in industry. 


\title{
Session 2aNSc
}

\section{Noise, NOISE-CON and Architectural Acoustics: International Noise Standards}

\author{
Paul D. Schomer, Chair \\ U.S. Army Construction Engineering Research Laboratory, P.O. Box 9005, Champaign, Illinois 61826-9005
}

Chair's Introduction-7:25

Invited Papers

$7: 30$

2aNSc1. The Acoustical Society of America program in standards. Paul Schomer (USA-CERL-CNN, P.O. Box 9005, Champaign, IL 61826-9005, schomer@uiuc.edu)

The Acoustical Society of America (ASA) Standards Secretariat has the delegated authority of the American National Standards Institute (ANSI) to manage the development of national standards, to provide for the USA input to the development of international standards, and to manage the development of certain international standards. First, the ASA Standards Secretariat manages the four " S' committees that develop ANSI Standards (national standards) using the consensus method. The four S-committees include S-1 (acoustics), S-2 (mechanical vibration and shock), S-3 (bioacoustics), and S-12 (noise). Second, the ASA Standards Secretariat manages the US representation to several International Organization for Standardization (ISO) and International Electro-technical Commission (IEC) technical committees including ISO TC 108 and 6 of its subcommittees, ISO TC-43 and TC-43-subcommittee 1, and IEC TC 29. Liaison representation from the Society of Automotive Engineers (SAE) and the American Society for Testing and Materials (ASTM) is relied on, in part, to perform this function. Third, the ASA Standards Secretariat provides the international secretariat for ISO TC-108 and two of its subcommittees. This paper discusses the role and program of the ASA Standards Secretariat.

2aNSc2. Work programs and interrelations of IEC/TC29, ISO/TC43 and ISO/TC43/SC1. Klaus Brinkmann (Physikalisch-Technische Bundesanstalt Braunschweig, Bundesallee 100, 38116 Braunschweig, Germany, klaus.brinkmann@ptb.de)

IEC/TC29 "Electroacoustics" and ISO/TC43 “Acoustics," together with its Subcommittee ISO/TC43/SC1 "Noise," each have very distinctive scopes. IEC/TC29 is mainly responsible for specification of performance characteristics of electroacoustical measuring instruments such as microphones, sound-level meters, sound calibrators, and audiometers, while ISO/TC43 and its SC1 primarily deal with measuring methods in audiology and noise control. Close links are, however, established by basically the same procedure for developing standards used in IEC and ISO, by common interest and mutual stimulation with respect to the instrumentation used for acoustical measurements and by overlapping involvement of experts in all three committees. The program of work of each committee will be briefly reviewed. Most actual information will be provided on IEC/TC29, which will have had its meeting just the week before. ISO/TC43 and ISO/TC43/SC1 will meet the following week, thus only allowing a forecast on expected achievements.

\section{8:30}

2aNSc3. Current trends in the international standardization of mechanical vibration and shock. Bruce E. Douglas (ISO Tech. Committee on Mech. Vib. and Shock and CSO, Resonance Technologies, 3731 Parke Dr., Edgewater, MD 21037)

Current concerns on world trade (e.g., fair business practices, product quality, and reliability), the environment (e.g., the propagation and radiation of noise from structures), and public safety (e.g., the effects of shock and vibration on humans, civil structures, and vehicles) have placed increased international attention on the development of quality standards in the field of mechanical vibration and shock. Recognizing its importance, the International Organization for Standardization (ISO) has set up a Technical Committee to develop standards in the field of mechanical vibration, shock, and the condition monitoring of machines and structures, ISO/TC 108 . Its scope includes the areas of mechanical vibration and shock pertaining to: terminology and nomenclature; actuators, sensors, and associated instrumentation; reduction and control methods; measurement and evaluation of the exposure of humans, stationary structures, vehicles, and machines. In addition, standardization in the field of vibration and shock for data processing, data acquisition, diagnostic measurement methods, transducer calibration, and condition monitoring are actively being developed. This presentation addresses topics on the broad issues of standardization in the field of mechanical vibration and shock with emphasis on (1) societal objectives, (2) economic stakes of diverse constituencies, and (3) structure, process, and future direction of ISO/TC 108. 
2aNSc4. Noise control and the machinery safety standardization program of CEN (European Standardization Committee). Jean R. Jacques (INRS, 30 rue Olivier Noyer, 75680 Paris, cedex 14, France), Roger F. Higginson (Higginson Acoust. Ltd., Bracknell, Berkshire RG129JL, UK), and Patrick Kurtz (BAuA, 44149 Dortmund, Germany)

One major aspect of the European internal market is the free circulation of products with a high level of safety. The European directive on machinery safety includes requirements to design low-noise machinery and to give out quantitative information on noise emission. One way for a manufacturer to get presumption of conformity to the directive is to use so-called harmonized European standards. In order to be harmonized and regarding noise, standards in the CEN machinery program (about 800 work items, each of them corresponding to a particular machinery family) must contain clauses on noise control and noise declaration, and a noise test code based on EN ISO 12001. Noise test codes specify the basic standards to be used for determining the emission sound pressure levels at workstations (among the EN ISO 11200 series) and the sound power level (among the EN ISO 3740 and 9614 series) and for declaring noise emission values (EN ISO 4871), together with the position of workstation(s) and the mounting and operating conditions of a machine during noise measurement. As part of a quality control system, noise consultants are employed by CEN to assess the noise contents of drafts and to assist Working Groups with noise matters.

9:10

2aNSc5. Building acoustic ISO standards and European legal metrology. Hans Goydke (PTB, Bundesallee 1000, 38114 Braunschweig, Germany)

Facilitating trade in the European Union through technical harmonization of products is the purpose of the Construction Product Directive of the European Commission. An appropiate tool to be expected is the development of harmonized international standards which have to be published as national standards in every member state. The cooperation of ISO and CEN succeeded in fulfilling the task, meanwhile, in the field of building acoustics with only a few exceptions. But, before these standards are to be incorporated into the legislation in the countries, for instance regarding legal requirements on the protection against noise in buildings, it has to be verified that the application of these standards shows sufficiently corresponding results when a test object is tested in laboratories in different countries. The results of such tests available, meanwhile, are partly unsatisfying. In order to solve the problems, the development of normative additions to some of the standards, for instance in order to specify more strictly mounting conditions of test objects, are most important work items of actual building acoustics standardization.

\section{9:30}

2aNSc6. ASTM Committee E-33 and its role in acoustical and noise standardization. Steven M. Brown (Steelcase, Inc., CD-5E-16, 6100 East Paris S.E., Caledonia, MI 49316, sbrown@scrbd.com)

ASTM Committee E-33 on Environmental Acoustics develops standards on the characteristics and performance of materials, products, systems, and services relating to the acoustical environment and the promotion of related knowledge. The Committee holds jurisdiction for over 50 standards on sound absorption, open plan spaces, sound transmission, the application of acoustical materials and systems, acoustical research, acoustical terminology, mechanical and electrical system noise, and community noise. Among these are the principal building acoustics standards used in North America and other areas. The Committee also provides the U.S. Technical Advisory Group for ISO/TC 43/SC 2 on Building Acoustics. This talk describes and explains E-33's activities and its relationship with other standards development organizations.

9:50

2aNSc7. SAE transportation noise activities. Richard F. Schumacher (GM Proving Ground, 3300 GM Rd., MC 483-356-000, Milford, MI 48380-3726) and Paul R. Donavan (GM Proving Ground, Milford, MI 48380-3726)

The SAE Vehicle Sound Level Form Committee continues to ensure the SAE road vehicle standards are maintained. Currently, there is a trend to move to internationally recognized standards that are technically valid procedures for rating the various products or subsystems. Although SAE has not initiated a development program, the light-vehicle standards group is actively reviewing an ISO project to develop more representatives of urban driving operations. All proposals are being evaluated to ensure that the procedure accommodates all vehicle designs including the US-style electronic-controlled automatic transmissions. An SAE horn and an accessory component committee are both actively involved in updating or developing new standards. SAE, while not involved in any major new initiatives, provides a significant role in review of world procedures. The expansion of our industrial world boundaries requires a continuing adjustment of our priorities to ensure we maintain standards that are relevant and provide the US-based industry an equal opportunity to be active participants in the global markets.

\section{0:10-10:30 Break}

\section{Contributed Papers}

\section{0:30}

2aNSc8. Standards in acoustics of the American Society of Heating, Refrigeration, and Air-Conditioning Engineers (ASHRAE). Richard J. Peppin (Scantek, Inc., 916 Gist Ave., Silver Spring, MD 20910, peppinr@asme.org)

This paper presents the extent of ASHRAE's involvement in sound and noise standards. ASHRAE has only a limited number of formal standards in acoustics but its two volumes of the four-volume set of hand- books contain many items that have become de facto standards. The "Fundamentals Handbook" and the "Applications Handbook" provide tables that show the power loss due to end reflection, attenuation of lined ducts, room criteria approaches and metrics, and other material properties that are used even though, and in spite of the fact that, they never were evaluated as a standard by Canvass or other means. I will summarize the topics in the ASHRAE publications and discuss the limitations and current controversial topics. 
10:45

2aNSc9. Environmental noise: From guidelines to standards. Dietrich Schwela (World Health Organization, Occupational and Environ. Health, 20 Ave. Appia, CH 1211 Geneva 27, Switzerland)

In view of the new WHO guidelines for Community Noise, a concept is proposed on deriving environmental noise standards on the basis of response-exposure relationships. WHO's guidelines have set definite guideline values for specific health effects and for specific environments. The WHO guidelines emphasize that exposure-response relationships between politically relevant variables (such as noise-induced social handicap, reduced productivity, decreased performance, absenteeism, drug use, and accidents) could definitely help decision makers to set sensible standards. In the standard-setting procedure, five different risks occur as part of any decision on the value of a standard. Three of these arise from the generalization of statements on susceptible sub-populations to be protected, on adverse effects of noise, and on boundary conditions of epidemiological studies. These risks cannot be quantified. One of the remaining two quantifiable risks is that of wrong measurements, which results from the quality of measurement techniques. The fifth risk relates to the acceptable probability of the occurrence of effects due to noise. With respect to the latter, governments have to fix an acceptable risk value in the standards-setting process. The consequences of combining these risks to a total risk are discussed.

\section{1:00}

2aNSc10. Noise impact from motor racing events. Alberto de Leo, Franco Giuliani, and Concetta Fabozzi (Natl. Agency for Environ. Protection of Italy (ANPA), Via V. Brancati 48, 00144 Rome, Italy)

The new Italian rules about noise pollution (General Law no. 447/95 and subsequent decrees) have caused the necessity of carrying out some measurement campaigns in order to observe the acoustic effects of various events and their interaction with territory and population. One of the re-

11:30-12:00

Panel Discussion search studies carried out by ANPA (Italian Agency for Environmental Protection) has dealt with a cognitive survey in motor racing sites and the noise impact that such infrastructures have on the surrounding environment. Measures have been done in and around motordromes, of which two are used for $F 1$ races and one for motorcycles races; they differ from one another for territory orography, wind conditions and outskirts urbanization.

\section{1:15}

2aNSc11. Problems of evaluation of long-term levels for Corona noise. Tadeusz Wszolek (Univ. of Mining and Metallurgy, Al. Mickiewicza 30, 30-059 Krakow, Poland)

The ac power lines are sources of audible noise (AN), emitted as a result of Corona effect, the intensity of which depends mainly on the weather conditions. Emitted AN mostly varies between 35 and $55 \mathrm{~dB}(\mathrm{~A})$ at 30-m distance. Corona AN may be considered to be composed of two major components: the low-frequency pure tones and the broadband noise. Whereas the broadband component is more or less stable, the tonal components are subject to great fluctuations. Therefore, their measurement presents certain difficulties. On the other hand, the tonal components are, according to the ISO 1996, accounted for in the AN evaluation by adding a correction to the measured level. Because of seasonal variation of the weather, long-term AN level values are taken for the evaluation, according to the ISO 1996. There is a problem concerning the evaluation of the actual periods of fair and bad weather conditions and their influence on the measurement result. The problem is also not solved by introducing the day/night levels (IEEE Std. 656). Another problem regards the environmental interference. In the paper the effect of possible errors, connected with the above-mentioned problems, on the final results of the long-term level calculations is analyzed.

\title{
Session 2aSA
}

\section{Structural Acoustics and Vibration, Signal Processing in Acoustics and Physical Acoustics: Diagnostics of Vibration and Noise in Structures I}

\author{
Sean F. Wu, Chair \\ Department of Mechanical Engineering, Wayne State University, 505 Anthony Wayne Drive, Detroit, Michigan 48202
}

Chair's Introduction-8:10

Invited Papers

8:15

2aSA1. Regularization of inverse problems with evanescent waves. Earl G. Williams (Naval Res. Lab., Code 7137, Washington, DC 20375)

Due to the presence of evanescent waves, acoustic and electromagnetic radiation problems form a class of ill-posed inverse problems when the vibration (electric currents) is to be determined solely from a near-field measurement of the pressure (electric field), as is done in near-field acoustical holography. The problem is ill posed since small changes in the measured pressure lead to large changes in the reconstructed velocity. Regularization of the problem is crucial. We will discuss various regularization methods which have been tested against experimental data and show how they can be modified and tuned in an effort to incorporate the physics of the decaying fields into them. In this way, we hope to identify a single technique which provides near optimum inversion of the ill-posed problem. [Work supported by ONR.] 
2aSA2. Reconstruction of acoustic radiation from an arbitrarily shaped object using the CHELS method. Sean F. Wu and Xiang Zhao (Dept. of Mech. Eng., Wayne State Univ., 5050 Anthony Wayne Dr., Detroit, MI 48202)

The combined Helmholtz equation-least-squares (CHELS) method developed previously by the authors [J. Acoust. Soc. Am. 107, 2799 (2000)] is used to reconstruct the acoustic field radiated from an arbitrarily shaped object. The main advantage of the HELS method [Wang and Wu, J. Acoust. Soc. Am. 102, 2020-2032 (1997); Wu, ibid. 107, 2511-2522 (2000)] is its simplicity in expressing the radiated acoustic pressure in terms of an expansion of certain basis functions, whose coefficients are determined by matching the assumed-form solution to the measured acoustic pressures. The number of expansion functions determines that of the measurements, which is small for structures that can be described by spheroidal coordinates. Combining the HELS method with the Helmholtz integral formulation will further allow for reconstruction of acoustic radiation from an arbitrarily shaped structure. This CHELS method is applied to a four-cylinder engine block. The normal component of surface velocity is reconstructed using singular value decomposition and a regularization technique. The corresponding surface acoustic pressure is obtained by solving the Helmholtz integral equation. Once these surface quantities are specified, the normal component of time-averaged acoustic intensity can be determined. Results of this study demonstrate that the CHELS method can become potentially a powerful noise and vibration diagnostic tool for practicing engineers. [Work supported by NSF.]

9:15

2aSA3. Analyzing structure radiation with eigenfunction expansions. J. D. Maynard (Dept. of Phys., The Pennsylvania State Univ., 104 Davey Lab., University Park, PA 16802)

A first step in noise control is to locate the regions on a structure which strongly couple sound energy into the surrounding medium. A powerful tool for accomplishing this is near-field acoustic holography, which can produce a map of the acoustic energy flow surrounding a structure. In the most manageable form of holography, reconstructions of the surface of the source can be obtained if the surface coincides with a level surface of a separable coordinate system, a severe limitation. For treating sources with arbitrarily shaped surfaces a new technique, using spherical wave function expansions and singular value decomposition, was developed at Penn State University. This technique avoids the evanescent waves of plane wave expansions. Computer implementations have become feasible with the availability of high-speed computers. A successful application of the new technique was an explanation of the measured radiation damping of elastic solids vibrating in a high-density gas. [Research was supported by National Science Foundation DMR 9801844 and the US Office of Naval Research.]

\section{$9: 45$}

2aSA4. Optimal regularization and resolution limits for the reconstruction of acoustic source strength by inverse methods. Philip A. Nelson and Youngtae Kim (ISVR, Univ. of Southampton, Highfield, Southampton SO17 1BJ, England)

Generalized cross validation (GCV) provides an effective method for the determination of optimal regularization parameters in acoustical inverse problems. The problem of reconstructing acoustic source distributions from field measurements is very often ill-posed.The use of Tikonhov regularization, for example, often suppresses the effect of small singular values in the Green function matrix to be inverted and these are in turn often associated with "high spatial frequencies" of the source distribution. The net effect is to produce a useful estimate of the acoustic source strength distribution but with a limited spatial resolution. This paper will explore the relationship between estimation accuracy, spatial resolution, noise level, and source sensor geometry when a range of inverse sound radiation problems is regularized using GCV. [Youngtae Kim is supported by a British Council Grant which is gratefully acknowledged.]

\section{0:15-10:30 Break}

\section{Contributed Papers}

10:30

2aSA5. An outline of a proposed acoustic holography system based on the Helmholtz equation-least-squares (HELS) method. Nassif E. Rayess and Sean F. Wu (Dept. of Mech. Eng., Wayne State Univ., Detroit, MI 48202)

The Helmholtz equation-least-squares (HELS) method [Wang and Wu, J. Acoust. Soc. Am. 102, 2020-2032 (1997); Wu, J. Acoust. Soc. Am. 107, 2511-2522 (2000)] has been proven a reliable acoustic holography method. The main advantages are its simplicity in formulations, flexibility in measurement locations, and uniformity in algorithm for a variety of source geometry and conditions. This paper presents a complete acoustic holography system based on the HELS method that makes full use of these advantages. This system has been under development as part of an ongoing research at the Acoustics, Vibration, and Noise Control Laboratory at Wayne State University. The physical setup will be described which includes the front-end multichannel data acquisition system and the associated hardware. Experimental results on reconstruction of the radiated acoustic fields from a complex source using this system will be demonstrated. Comparisons of this system with other acoustic holography systems currently available will be made. [Work supported by NSF.]

\section{0:45}

2aSA6. Why can spherical harmonics be used to describe the sound field generated by nonspherical sources? Nassif E. Rayess and Sean F. Wu (Dept. of Mech. Eng., Wayne State Univ., Detroit, MI 48202)

The spherical harmonics in conjunction with the spherical Hankel functions form a complete set of spherical basis functions, which can fully describe the radiated sound field from a vibrating object. The vibrating object itself need not be spherical as long as the exterior region is beyond a minimum sphere that completely encloses the object. Such expansion solutions are not supposed to be valid inside the minimum sphere. However, experimental results [Rayess and Wu, J. Acoust. Soc. Am. 107] using the Helmholtz equation-least-squares (HELS) method [Wang and $\mathrm{Wu}$, J. Acoust. Soc. Am. 102] have shown that a modified set of spherical basis functions can accurately describe the sound field well inside the minimum sphere. That modified set is obtained by Gram-Schmidt orthonormalization of the spherical basis functions with respect to the source surface. It is shown that the velocity fields corresponding to these modified basis functions form an orthogonal and complete set, which can uniquely describe the velocity field on the surface. The proof given in this paper 
will provide a solid foundation for the establishment of the HELS method as a potentially viable noise diagnostic tool. [Work sponsored by NSF and Ford Motor Company.]

\section{1:00}

2aSA7. Determination of the optimal number of expansion terms in the HELS method. Byoung-Duk Lim (Dept. of Mech. Eng., Yeungnam Univ., 214-1 Dae-dong, Gyongsan, Kyungbuk, 712-749, Korea) and Sean F. Wu (Wayne State Univ., Detroit, MI 48202)

The Helmholtz equation least-square (HELS) method has been proven to be an efficient way of reconstructing acoustic radiation from a vibrating structure [Wu, J. Acoust. Soc. Am. 107, 2511-2522 (2000)]. In this method, the optimal number of expansion terms is determined by a heuristic method of searching the minimum-norm errors of the reconstructed acoustic pressures with respect to the measured data. While this approach works, it is time consuming. In this paper, a new method of a priori determination of the optimal number of expansions is developed based on the eigenvalue analysis of the measured information. Assuming a Gaussian white measurement noise, the hypothesis of equal noise eigenvalues is tested based on the statistical properties of the eigenvalues. This hypothesis test yields an upper bound of the optimal number of expansions in general. Also developed is a method for testing the correlation coefficients after eliminating some principal components, which gives a lower bound of the optimum number of expansions. Since the curve of minimum-norm errors in the previous work shows local minima when the number of terms is squares of the integer, the range produced by the present method leads to only a few candidates for the optimal number of expansions. Examples of reconstructing the radiated acoustic pressure field from a vehicle front end using the optimal number of expansion terms thus obtained are demonstrated in parallel with the results of the a posteriori method discussed by Wu. [Work supported by NSF.]

\section{1:15}

2aSA8. Vibro-acoustography of small spheres. Shigao Chen, Mostafa Fatemi, and James F. Greenleaf (Dept. of Physiol. and Biophys., Mayo Clinic, Rochester, MN 55905, chen.shigao@mayo.edu)

Vibro-acoustography is a method for imaging the acoustic energy emitted from objects in response to an oscillatory radiation force produced by two interfering focused beams of ultrasound [M. Fatemi and J. F. Greenleaf, Science 280, 82-85 (1998)]. To facilitate quantitative study, a model is presented that describes the behavior of an elastic sphere in two plane ultrasound waves of slightly different frequencies. The vibrating velocity and the resulting acoustic emission of the sphere at the difference frequency are derived from the radiation force and the impedance of the sphere. For small steel spheres in water, the velocity and emission are predicted to have a simple relationship with the difference frequency and the size of the sphere. Experiments on small stainless steel spheres of different size are carried out for a number of difference frequencies, with a laser interferometer to measure the velocity of the sphere and a cali- brated hydrophone to measure the acoustic emission from the sphere. The experimental results fit the model description well. This means that vibroacoustography may be useful in quantitative measurement.

\section{1:30}

2aSA9. Backscattering enhancements associated with antisymmetric Lamb waves on circular plates in water: Observations and imaging of the plate surface using acoustic holography. Brian T. Hefner and Philip L. Marston (Phys. Dept., Washington State Univ., Pullman, WA 99164, bhefner@mail.wsu.edu)

When a tilted circular plate is illuminated with high-frequency sound in water, significant enhancements occur when the angle of incidence corresponds to a Lamb wave coupling angle. Previously, scattering associated with the lowest-order symmetric Lamb wave, $s_{0}$, was studied using acoustic holography and modeled using quantitative ray methods $[\mathrm{B} . \mathrm{T}$. Hefner and P. L. Marston, J. Acoust. Soc. Am. 107, 2847 (2000)]. The present investigation focuses on backscattering associated with the antisymmetric Lamb waves. Above the cutoff frequency of the first-order antisymmetric wave, $a_{1}$, enhancements are observed which are associated with both the $a_{1}$ wave and the $a_{0}$ wave. For these enhancements, however, mode conversion between these modes plays a significant role. To examine the mode conversion and to identify the scattering mechanisms, acoustic holography was used to image the plate surface when the plate is ensonified at the $a_{1}$ wave coupling angle. From the imaged wave field, the enhancement at this angle involves a wave which moves around the circumference of the plate: a "whispering gallery" mode involving the $a_{1}$ wave or a flexural edge wave. Both of these possibilities are considered as well as a possible scattering mechanism at the $a_{0}$ wave coupling angle. [Work supported by the Office of Naval Research.]

\section{1:45}

2aSA10. Diagnostics of vibration and noise via Fourier transforms: A new approach. Leonid M. Gelman (Dept. of Nondestructive Testing, Natl. Tech. Univ. of Ukraine, 37, Peremogy Pr., Kiev, 252056, Ukraine), Ivan V. Petrunin (Natl. Tech. Univ. of Ukraine, Kiev, 252056, Ukraine), and Vladimir T. Shirkov ("Motor-Sich" Co., Zaporozh'e, 01202, Ukraine)

A new general optimal approach and new diagnostic features are proposed, for those cases where one- and multidimensional Fourier transforms are used for diagnostics of vibration and noise. For the forced oscillation vibroacoustical diagnostics method with Gaussian excitation, the optimal nonlinear transformation of the proposed features is received. It was shown (1) The power spectral density is optimal transformation for considered diagnostics only for specific situations; (2) The phase spectrum is not optimal transformation for considered diagnostics; and (3) The proposed approach provides the increment of diagnostics effectiveness in contrast with usage of power spectral density. This is in contrast to most applications concerning diagnostics of vibration and noise, where power spectral density is used. 
Session 2aSC

\title{
Speech Communication: Articulation (Poster Session)
}

\author{
Kristin K. Tjaden, Chair \\ Department of Communicative Disorders and Sciences, The State University of New York at Buffalo, 122 Cary Hall, \\ 3435 Main Street, Buffalo, New York 14214-3005
}

\section{Contributed Papers}

\begin{abstract}
All posters will be on display from 9:00 a.m. to 12:00 noon. To allow contributors an opportunity to see other posters, contributors of odd-numbered papers will be at their posters from 9:00 a.m. to 10:30 a.m. and contributors of even-numbered papers will be at their posters from 10:30 a.m. to 12:00 noon.
\end{abstract}

2aSC1. A cross-linguistic acoustic study of fricatives. Matthew K. Gordon, Paul Barthmaier, and Kathy Sands (Dept. of Linguist., Univ. of California, Santa Barbara, Santa Barbara, CA 93106)

This work presents results of an acoustic study of fricatives in 7 languages (Aleut, Chickasaw, Hupa, Montana Salish, Scottish Gaelic, Toda, and Western Apache), all of which contrast fricatives made at several places of articulation. Measurements of the frequency of spectral peaks and centroid frequencies indicate many similarities between the languages in the acoustic properties defining the fricatives. Some of the principal findings are the following. Alveolar sibilants typically have the highest spectral peak and centroid frequency. Lateral and palatoalveolar fricatives have spectral peaks and centroids intermediate in frequency between alveolar sibilants and backer fricatives. Among the back fricatives, peaks and centroids of uvulars are characteristically lower than those of velars. Rounding of back fricatives induces further lowering of peaks and/or centroids. Contrasts in backness and rounding among the back fricatives are also associated with differences in $F 2$ of the following vowels: $F 2$ values are lower following uvulars than velars, and lower following rounded than unrounded fricatives. Labiodental fricatives typically have flat spectra with poorly defined spectral peaks. Finally, the contrast between lateral fricatives and palatoalveolar sibilants is variably realized, depending on language and speaker, as a difference in the location of spectral peaks and/or centroid frequency.

2aSC2. The noise spectrum characteristics of retroflexed consonants in Mandarin Chinese. Jing-Yi Jeng (Dept. of Special Education, Natl. Tainan Teacher College, Taiwan, ROC)

Retroflexed consonants are the most complex and difficult sounds for Mandarin speakers. The four retroflexed consonants in Mandarin are produced with the tip of the tongue retracted and curled up close to the positions of the hard palate. The purpose of this study was to investigate the acoustic characteristics of retroflexed consonants for normal speakers. The moment analysis and the noise duration of retroflexed and nonretroflexed fricatives and affricates were studied for 10 adult speakers. Moment analysis is a statistical approach for the power spectrum. The results showed that retroflexed consonants had lower mean noise frequencies (M1) than nonretroflexed consonants. The standard deviation of noise energy frequencies (M2) of retroflexed consonants was smaller than those of nonretroflexed consonants. Results will be discussed in terms of the linkage between acoustic data and tongue positions. The discriminate analysis by using moment and duration parameters to classify word-initial retroflexed consonants was also reported.
2aSC3. New effects of laryngeal configurations on $f 0$ : Voiceless stops in Korean. Sunyoung Oh (Dept. of Linguist., Univ. of British Columbia, E270-1866 Main Mall, Vancouver, BC V6T 2E1, Canada, sunyoh@interchange.ubc.ca)

Korean has three sets of voiceless stops in word-initial position which are distinctive from one another in terms of laryngeal adjustments: lenis unaspirated, fortis unaspirated, and aspirated [P. Ladefoged and I. Maddiedson, 47-101 (1996)]. It has been observed that, in Standard Korean, the aspirated stops have higher $f 0$ value than the fortis stops, while both stops have higher $f 0$ value than the lenis stops [D. Silva, 11-34 (1998)]. It could be argued that this indicates a universal correspondence between laryngeal configurations for voicing and pitch—at least within Korean. However, the current paper reveals a different observation. In South Kyengsang dialect in Korean, little difference is found in the $f 0$ value between the aspirated and the fortis stops, while the $f 0$ of the lenis stops is relatively lower, similar to Silva's (1998) finding. A total of 600 tokens, by five speakers, were recorded and digitized, then analyzed using Macquirer software for pitch tracking. The results show that the $f 0$ following the fortis stops are consistently higher than that of Standard Korean, regardless of the place of articulation, and the higher $f 0$ of the fortis stops patterns with the lower $f 0$ of the lenis stops among speakers.

2aSC4. Acoustic evidence of a phonetics-phonology mismatch in Nuu-chah-nulth. Ian L. Wilson (Dept. of Linguist., Univ. of British Columbia, Buchanan E270-1866 Main Mall, Vancouver, BC V6T 1Z1, Canada)

Measurements were made of the acoustic properties of the pharyngeal $\mathrm{S}$ in Ahousaht Nuu-chah-nulth (Nootka), an endangered language spoken on Vancouver Island. One goal of the study was to test whether phonetics and phonology make the same predictions regarding the classification of $\mathrm{Y}$. Although the phonological data largely favors an analysis of $\uparrow$ as a type of glottalized pharyngeal stop, the results of acoustic measurements suggested that it is a glottalized glide. These acoustic measurements included a comparison of the waveforms and spectrograms of $\uparrow$ to those of glottalized resonants and glottalized stops. Three types of acoustic evidence were found to support the claim that $\subseteq$ is a glottalized glide. Specifically, the timing of glottalization in $\subseteq$ was like that of a glottalized resonant, rather than a glottalized stop; in several tokens full stop closure was not achieved; and the duration of the formant transition of $\subseteq$ is more like that of a glide than a stop. This acoustic evidence helps to illustrate one example of a phonetics-phonology mismatch, and the fact that there is a mismatch supports a modular view of grammatical organization. [Work supported by SSHRCC and NSERC Grants.] 
2aSC5. Cues for place and palatalization in Russian stops. Colleen Richey (Dept. of Linguist., Stanford Univ., Margaret Jacks Hall Bldg. 460, Stanford, CA 94305-2150)

Using acoustic measurements and native speaker perceptions, the cues for place of articulation of Russian plain and palatalized stops were investigated. The acoustic measurements were made of formant transitions, burst duration, and spectral burst shape. They showed that formant transitions distinguish place of articulation of plain consonants, but not palatalized consonants. Differences distinguishing place of articulation were found in the burst durations and in the ratio of energy between the upper and lower portions of the burst spectra. In the perception experiment, native Russian speakers listened to various consonant-vowel stimuli. Hearing only the formant transitions, listeners were able to identify place of articulation of just the plain stops. When they heard stimuli with conflicting cues for place in the burst and formant transitions, in the majority of the cases, they identified plain consonants based on the formant transitions, but they identified palatalized consonants based on the burst. It is concluded that while formant transitions do provide the strongest cues for place among plain stops, they do not provide cues for place among palatalized stops. The cues for place of articulation of palatalized consonants are contained completely in the duration and spectral shape of the burst.

2aSC6. Dialect differences in the temporal characteristics of vowels: A comparison of standard (Parisian) and Swiss French. Joanne L. Miller, Michèle Mondini (Dept. of Psych., 125 NI, Northeastern Univ., Boston, MA 02115, jlmiller@neu.edu), François Grosjean (Univ. of Neuchâtel, Switzerland, francois.grosjean@lettres.unine.ch), and JeanYves Dommergues (Univ. of Paris, 7, France)

Earlier we reported a dialect difference in the use of temporal information for vowel perception: Native speakers of Swiss French used temporal as well as spectral information when identifying /o/ versus $/ 0 /$, whereas native speakers of standard (Parisian) French used only spectral information [J. L. Miller and F. Grosjean, Language Speech 40, 277-288 (1997)]. We interpreted this dialect difference in terms of the more prominent role that vowel duration plays overall in the phonological system of Swiss French compared to standard French. To investigate further the basis of the dialect effect, we have been measuring the duration of $/ \mathrm{o} /$ and $\mathrm{s} / \mathrm{in}$ monosyllabic words for native speakers of the two dialects. Our findings to date indicate a robust dialect effect in production: The duration difference between $/ 0 /$ and $/ \mathrm{J} /$ is substantially larger and more consistent in Swiss French than in standard French. Thus the perceptual dialect effect for $/ 0 /$ and $/ 2 /$ we reported earlier reflects both a specific difference in the temporal characteristics of this vowel pair and an overall difference in the role of vowel duration in the phonological systems of the two dialects. [Work supported by NIH/NIDCD, Swiss NSF.]

2aSC7. Gemination in Italian: the GEMMA project. Maria-Gabriella Di Benedetto (Univ. of Rome La Sapienza, Infocom Dept., via Eudossiana 18, 00184 Rome, Italy, gaby@acts.ing.uniroma1.it)

The Gemination project (GEMMA) started at the University of Rome La Sapienza, Italy, in 1992, examined gemination in Italian. The analyzed consonants were stops, liquids, fricatives, nasals, and affricates. GEMMA was completed in 2000. Partial results were previously reported [stops: Esposito, DiBenedetto (1999), liquids: Argiolas et al. (1995), fricatives: Giovanardi, DiBenedetto (1998), nasals: Mattei, DiBenedetto (2000), affricates: Faluschi, DiBenedetto (2000)]. The major findings were coherent in all studies: gemination is revealed by time-related parameters, namely, the consonant lengthening and the preconsonant vowel shortening in geminate forms. Relevance of acoustic correlates was confirmed by perceptual experiments [A. Esposito and M. G. DiBenedetto, J. Acoust. Soc. Am. 106, 411-422 (1999); F. Argiolas et al., J. Acoust. Soc. Am. 97, 3418 (1995); M. Giovanardi and M. G. DiBenedetto, Eur. J. Language
Speech 1-13 (1998), http://web-sls.essex.ac.uk/web-sls; M. Mattei and M. G. DiBenedetto, Eur. J. Language Speech 1-14 (2000), http://websls.essex.ac.uk/web-sls; S. Faluschi and M. G. DiBenedetto, Eur. J. Language Speech (submitted)].

2aSC8. Effects of speaking rate on vowel formant trajectories. Jeff Berry and Gary Weismer (Dept. of Communicative Disord. \& Waisman Ctr., Univ. of Wisconsin—Madison, Goodnight Hall, Madison, WI 53706, weismer@waisman.wisc.edu)

The effects of speaking rate and vowel context on formant frequencies have traditionally been assessed by analyzing the variation in some operationally defined "target" frequency value. The use of a single frequency value to describe the behavior of a formant has proven useful for distinguishing among vocalic nuclei and identifying "target undershoot" effects of speaking rate. We have, however, only limited understanding of the characteristic effects of speaking rate change on formant trajectories. In our work, analysis of more complete representations of the timevarying changes in formant frequencies throughout vocalic nuclei have revealed patterns of formant change across speaking rates that differ qualitatively and quantitatively between speakers. These data suggest speakerspecific strategies for accomplishing speaking rate change. We have also observed that the acoustic effects of rate change within speakers differ qualitatively and quantitatively between different ranges of the vowel duration continuum. Thus, the acoustic effects of small changes in speaking rate at speaking rate extremes are not comparable to quantitatively identical changes at habitual rates. Since changes in speaking rate are commonly thought to correspond with changes in the overlap of neighboring articulatory gestures, these findings are pertinent to modeling coarticulation. [Work supported by NIH DC 0149-10.]

2aSC9. Spectral characteristics of $/ \mathrm{s} /$ and $/ \int /$ : Rate effects in Parkinson's disease. Kris Tjaden, Jessica Kleinhaut, and Alexa Kozak (Dept. of Communicative Disord. and Sci., The State Univ. of New York at Buffalo, 3435 Main St., Buffalo, NY 14214-3005)

Comtemporary speech production theory proposes that speakers adjust rate to enhance or maximize acoustic-perceptual distinctiveness for listeners. In fact, the suggestion that rate impacts acoustic-perceptual distinctiveness is one rationale for using rate manipulation as a therapy to improve speech intelligibility in dysarthria. The relationship between rate manipulation, acoustic measures of production, and perceptual impressions of speech in dysarthria is poorly understood, however. For example, some studies report rate-induced acoustic changes in speech that are systematically tied to perceptual impressions. Other studies report no effect of rate on speech production characteristics in dysarthria. Further study of the relationship between speech production measures and rate variation in dysarthria therefore appears warranted. The present study examined spectral characteristics of word-initial /s/ and / $/$ / produced at a variety of rates by speakers with hypokinetic dysarthria associated with Parkinson's disease. Healthy speakers were studied for comparison purposes. Fricatives were produced in the words shores, shies, size, and soys embedded in a carrier phrase. A graded rate task was used to elicit an extensive range of rates. Spectral moment coefficients were used to characterize fricatives; phrase duration was used to index rate. The relationship between rate variation and spectral moment coefficients was quantified via regression analysis.

2aSC10. An evaluation of phonetic working space in normal geriatrics and persons with motor speech disorders. Kate Bunton, John Westbury, and Gary Weismer (Waisman Ctr., Univ. of WisconsinMadison, 1500 Highland Ave., Madison, WI 53705, bunton@waisman.wisc.edu)

Speakers with dysarthria seem to have smaller than normal phonetic working spaces. Their vowels exhibit unusual and often reduced formant transitions and slopes, and areas enclosed by their "vowel locations" plotted in an $(F 1, F 2)$ plane are abnormally small. Presumably, reduced 
formant transitions reflect small movements of individual articulators. Reduced lip and jaw movements have been reported for speakers with Parkinson's disease and amyotrophic lateral sclerosis. However, comparable reports for tongue movement are not available. In this study, $\mathrm{x}$-ray microbeam data were used to compare the acoustic and articulatory phonetic working spaces of neurologically normal and dysarthric speakers. Measurements of formant frequencies, and sagittal-plane locations of tongue fleshpoints, were made for the English vowels /i ae a u/ at the temporal midpoint of the vowel nucleus in three different words for each of ten normal and seven dysarthric speakers. Speakers' articulatory phonetic working spaces were defined as areas enclosed by tongue fleshpoint locations. Data obtained from this study provide an enhanced appreciation of the articulatory bases of acoustic features of dysarthric speech. [Work supported by 1-R01-DC03723.]

2aSC11. Observing the link between speech production and speech perception from disordered speech. Emily Q. Wang (Dept. of Commun. Disord. and Sci., Rush Univ., 1653 W. Congress Pkwy., Ste. 203 SENN, Chicago, IL 60612), Yi Xu (Northwestern Univ., Evanston, IL 60208), Katie Kompoliti, and Christopher G. Goetz (Rush Univ., Chicago, IL 60612)

It was recently reported that individuals with idiopathic Parkinsons Disease (PD) and hypokinetic dysarthria were able to signal sentence focus using acoustic parameters such as $f 0$, intensity, and vowel duration that normal speakers use. However, it was also found that PD speakers did not show all the $f 0$ patterns that normal speakers use. For example, although the $f 0$ range of the word under focus was expanded, the $f 0$ range of the postfocus word was not always suppressed. In this paper, the $f 0$ patterns produced by ten speakers with idiopathic PD (Hoehn and Yahr stage 3 and 4, off) and mild, or mild-moderate, or moderate hypokinetic dysarthrias will be examined. These patterns will also be correlated to their perception by normal speakers. The following issues will be addressed: (1) Can listeners correctly perceive focus produced by the PD speakers when the $f 0$ patterns are similar to those used by normal speakers? (2) Can listeners correctly perceive focus when there are missing cues in the $f 0$ patterns (e.g., lack of post-focus suppression)? (3) Can misperception result from the speakers attempt to compensate for their articulatory limitations due to the disease process? Implications of the findings will be discussed in terms of speech motor control.

2aSC12. On the correlation between articulatory and acoustic data. Jintao Jiang, Abeer Alwan (Elec. Eng. Dept., Univ. of California, Los Angeles, Los Angeles, CA 90095), Patricia Keating (Univ. of California, Los Angeles, Los Angeles, CA, 90095), Lynne E. Bernstein, and Edward Auer (House Ear Inst., Los Angeles, CA 90057)

In J. Acoust. Soc. Am. 107, 2904 (2000), we investigated the correlations between external facial movements, tongue movements, and speech acoustics using a database of consonant-vowel syllables spoken by one female and two male talkers. The intelligibility of the talkers, based on visual information, was judged by hearing-impaired individuals. In this study, we extend the analysis to two sentences and a fourth talker (female); each sentence was repeated five times. Two of the talkers (one male and one female) had high-intelligibility ratings while the other two had low ratings. A Qualisys (optical motion capture system) and an EMA system were used to characterize orofacial and tongue movements, respectively. The articulatory and acoustic data streams were recorded simultaneously and aligned. Acoustic features were represented by line spectral pairs. A multilinear regression technique was applied to quantify the correlation between the data. A universal estimator was obtained with training data, using a jack-knife approach, and then applied to the test data. Correlations results were speaker dependent, and, on average, the highest correlations were between the optical and EMA data. Detailed results, including a comparison between the results for CVs and sentences, and implications will be discussed. [Work supported in part by NSF KDI award 9996088.]
2aSC13. Tongue surface dynamics during speech and swallowing. Jordan R. Green, Steven L. Pittelko, and Yutsai Wang (Dept. of Commun. Disord., Univ. of Wisconsin-Madison, 1975 Willow Dr., Madison, WI 53706)

This investigation characterizes tongue surface dynamics that underlie phonemic variation and that distinguish speech from swallowing. Vertical displacements of pellets affixed to the tongue were extracted from the x-ray microbeam database [Westbury, J. X-ray Microbeam Speech Production Database Users Handbook, Version 1 (1994)], which contains articulatory kinematic data from 57 typical speakers. Participants recited 21 vowel-consonant-vowel (VCV) combinations, read the Grandfather Passage, and swallowed $10 \mathrm{cc}$ of water. Consonantal context was manipulated in the VCV utterances as a means to describe the range of tongue dynamics produced during speech. For each task, tongue dynamics was quantified by performing zero-lag cross correlations on selected pellet pairs. A coupling index was then computed by scaling the derived coefficients by a multiplier that reflected the amplitude of each displacement signal. A wide range of movement coupling among tongue pellets was observed across tasks. Phonemic differentiation in vertical tongue dynamics was observed as coupling varied predictably across marker pairs with place of articulation. Moreover, tongue displacements for speech and swallowing clustered into distinct groups based on their coupling profiles. The strengths and weaknesses of the coupling index for characterizing tongue surface dynamics across multiple speakers are considered.

2aSC14. Vocal-tract model experiment for illustrating vowel production. Soo-Ki Hong and S. W. Yoon (Acoust. Res. Lab., Dept. of Phys., Sung Kyun Kwan Univ., Suwon 440-746, Republic of Korea)

Everyone can articulate the same words even though each individual has unique vocal organ. Findings of common features in the same vowels articulated by everyone are very useful for speech recognition and reproduction. In this study a vocal tract is modeled with a cylindrical silicone tube to understand the mechanisms of vowel production. The constrictions on the tube are varied with locations, lengths, and degrees. The resonance frequency shifts of vocal-tract depend on the length ratio and the crosssection area ratio of the constriction in the tube. The resonance frequencies show good correlation with the formants measured in speech. [One of the authors (SWY) was supported by the BK21 project.]

2aSC15. Simulations of voice onset-offset hysteresis in /aha/ utterances. Jorge C. Lucero (Dept. of Mathematics, Univ. of Brasilia, Brasilia DF 70910-900, Brazil, lucero@mat.unb.br) and Laura L. Koenig (New York Univ.)

The subcritical Hopf bifurcation model for voice onset-offset hysteresis [J. C. Lucero, J. Acoust. Soc. Am. 105, 423-431 (1999)] is investigated considering the glottal area as control parameter. A two-mass model of the vocal folds coupled to a two-tube approximation for the vocal tract is used for producing oral airflow simulations. The glottal rest area is varied in an abduction-adduction pattern to reproduce smoothed oral airflow records measured during /aha/ production [L. L. Koenig and R. S. McGowan, J. Acoust. Soc. Am. 100, 2689(A) (1996)]. The resultant simulations show good agreement with the unsmoothed measured records, with a clear hysteresis effect: vocal-fold oscillation stops during glottal abduction at a higher value of the glottal area than the value at which it starts during the adduction. The dependence of the onset-offset hysteresis and voice parameters on vocal-fold stiffness ( $Q$ factor), subglottal pressure, and male-female laryngeal configurations is analyzed and illustrated with bifurcation diagrams. [Work supported by CNPq, Brazil, and NIH.] 
2aSC16. An EPG study of allophonic variation in the gestural magnitude of English lingual consonants. Bryan Gick (Dept. of Linguist., Univ. of British Columbia, E270-1866 Main Mall, Vancouver, BC V6T 1Z1, Canada, gick@interchange.ubc.ca)

Previous work on English glides and liquids has shown that certain (consonant) gestures are most constricted in initial allophones, least constricted in final allophones, and constricted to an intermediate degree in ambisyllabic or resyllabified allophones. It is not known, however, whether this generalization applies to other consonants such as stops and fricatives. As the point-tracking and imaging techniques used in previous studies are not well suited for measuring these types of segments, the present paper will present the results of an EPG study testing the degree of tongue contact during lingual consonants in these three syllable positions. Results of a pilot study using two subjects and a reduced set of consonants were mixed, failing to replicate previous findings for some previously measured consonants (e.g., /n/), while supporting the predicted pattern for others not previously measured (e.g., /z/). Results will be presented for additional subjects using the full inventory of lingual consonants. [Work supported by NSERC.]

2aSC17. A study of compensation for a labial perturbation of the vowel / $\mathbf{u} /$ using an area function model of the vocal tract. Brad $\mathrm{H}$. Story (Dept. of Speech and Hearing Sci., Univ. of Arizona, P.O. Box 210071, Tucson, AZ 85721-0071, bstory@u.arizona.edu)

This study reports on the ability of an area function model of the vocal tract [Story and Titze, J. Phonetics 26, 223-260 (1998)] to compensate for a labial perturbation. The area function model, which consists of a neutral shape and two weighted orthogonal shaping patterns (modes), is first configured for a typical production of /u/. An effective lip tube based on the labial perturbation experiment reported by Savariaux et al. [J. Acoust. Soc. Am. 98, 2428-2442 (1995)] is then imposed on the area function model (by forcing the lip section to be a constant cross-sectional area). Frequency responses are computed for both the normal and the perturbed shapes. Next, an attempt was made to vary the weighting values for the two shaping patterns of the area function model so that the first two formants were moved back toward their unperturbed frequency locations. The result was that weighting values can be determined that do move the formants in the appropriate direction for compensation. Additionally, the resulting area function shows characteristics similar to those presented by Savariaux et al.; that is, a movement of the constriction location posteriorly and a widening of the anterior portion of the front cavity. [Work supported by NIH R01-DC04789-01.]
2aSC18. Electropalatographic and acoustic measures of adaptation to palatal perturbation. Wendi A. Aasland, Shari R. Baum (School of Commun. Sci. \& Disord., McGill Univ., 1266 Pine Ave. W., Montreal, QC H3G 1A8, Canada, c3cr@musica.mcgill.ca), and David H. McFarland (Universite de Montreal, Montreal, QC, Canada)

The present investigation examined adaptation to a palatal perturbation in $[\mathrm{s}$ ] production, as reflected in both electropalatographic (EPG) and acoustic measures. The goal was to explore the development of compensatory motor programs during the production of individual fricative segments, as well as over a 1-h period of focused practice. Thirty repetitions of the syllable [sa] were produced at each of five time intervals (times 0 , $15,30,45$, and $60 \mathrm{~min}$ ) by a speaker wearing a specially designed pseudopalate with a $6 \mathrm{~mm}$ buildup of acrylic at the alveolar ridge. At times 0 and 60 , baseline measures of [sa] production with a thin pseudopalate and with no palate in place were also recorded to characterize unperturbed articulation. Acoustic analyses focused on centroid frequencies for the $[\mathrm{s}]$ productions, while a wide range of EPG analyses were conducted including measures of groove length and width, location and duration of maximum constriction, and variability in tongue-palate contact patterns throughout the fricative production. Preliminary analyses indicate a high degree of variability throughout the practice period. Results are discussed in relation to the development of adaptive articulatory programs in speech motor control and the articulatory configurations necessary for adequate [s] production. [Work supported by NSERC.]

2aSC19. The use of functional data analysis to study variability in children's speech. Laura L. Koenig (Dept. of Speech-Lang. Pathol. and Audiol., New York Univ. and Haskins Labs) and Jorge C. Lucero (Univ. of Brasilia)

A common finding in studies of children's speech is increased variability relative to adults. This result may be observed well after the age when basic phonological contrasts are established. Researchers debate the sources of this variability, but most agree that the study of variability, and its decrease over time, may provide insight into motor control development. Recent data on oral airflow during $/ \mathrm{h} /$ in 5 -year-olds and adults revealed that the children presented greater relative amplitude variability than the adults, and seemed to show less regularity in the rates of ad/ abduction for $/ \mathrm{h} /$. This presentation applies functional data analysis (FDA) to the airflow data from two women and one 5-year-old speaker. In FDA, signals are nonlinearly normalized using a smooth warping function of time, and separate indices are obtained for phasing (temporal) and amplitude variability. Results indicate that the child shows greater variability in both temporal and amplitude measures, but the extent of adult-child differences depends somewhat on the specific methods of normalization and alignment. FDA shows potential as a means of gaining more detailed information about the nature of variability in children's speech data. [Work supported by NIH and CNPq, Brazil.] 


\title{
Session 2aUW
}

\section{Underwater Acoustics and Acoustical Oceanography: High-Frequency Sediment Acoustics and Associated Sediment Properties and Processes I}

\author{
Eric I. Thorsos, Chair \\ Applied Physics Laboratory, University of Washington, 1013 NE 40th Street, Seattle, Washington 98105
}

Chair's Introduction-7:55

Contributed Papers

8:00

2aUW1. SAX99: An experiment in high-frequency sediment acoustics. Eric I. Thorsos (Appl. Phys. Lab., Univ. of Washington, Seattle, WA 98105)

In the fall of 1999, "SAX99" (for sediment acoustics experiment1999) was performed at a site $2 \mathrm{~km}$ from the shore on the Florida Panhandle in water $18-19 \mathrm{~m}$ in depth. The seafloor sediment was medium sand, and acoustic frequencies were mainly in the $10-300-\mathrm{kHz}$ range. A wave-induced ripple field was present at the site. The main goals in SAX99 were to quantify acoustic backscattering from the sediment, acoustic penetration into the sediment (above and below the critical angle of 30 deg), and acoustic propagation within the sediment. Extensive environmental characterizations were made at the experiment site. Quantities that enter into a Biot model description of the sediment were measured. Sediment variability was measured to centimeter scales, giving the most complete coverage for the frequency range of $10-50 \mathrm{kHz}$. An overview of the entire SAX99 measurement program will be given. The range of acoustic measurements will be briefly described, as well as the scope of measurements of the seafloor physical properties and of biological processes within the sediment and the water column. Initial results of this work will be described in separate papers. [Work supported by ONR.]

\section{$8: 15$}

2aUW2. Buried target detection using synthetic aperture sonar. Kerry W. Commander and John E. Piper (Coastal Systems Station, Code R21, 6703 W. Hwy. 98, Panama City, FL 32407-7001, commanderkw@ncsc.navy.mil)

During the Sediment Acoustics Experiment in 1999, the Coastal Systems Station fielded a synthetic aperture sonar (SAS) operating at $20 \mathrm{kHz}$. The SAS was able to detect and image $1.5 \times 0.5 \mathrm{~m}$ cylinders at burial depths up to $50 \mathrm{~cm}$ and grazing angles well below the critical angle. In order to clarify the effect of the rough surface (sand ripple field) on sound propagation into the sediment, an experiment was recently conducted using five 10-in. fluid-filled target spheres that were buried at depths ranging from 5 to $50 \mathrm{~cm}$ below the top of the spheres. The interior fluid was chosen to maximize the target strength of the spheres (approximately -11 $\mathrm{dB}$ ) when buried in sediment with a sound speed of $1750 \mathrm{~m} / \mathrm{s}$. Using aspect-independent targets enables the determination of the sound level transmitted into the sediment as a function of the angle between the transmitted acoustic beam and the sand ripple direction. Complete $360 \mathrm{deg}$ azimuthal patterns of target echo levels are presented, illustrating the variation due to transmit/receive azimuthal angle with respect to the ripple structure. These azimuthally dependent echo level patterns are presented for several incident grazing angles, all of which are subcritical. [Work supported by ONR Code 321TS.]
$8: 30$

2aUW3. Applied Physics Laboratory participation in SAX99: Experiment design and execution. Kevin L. Williams, Darrell R. Jackson, Dajun Tang, and Eric I. Thorsos (Appl. Phys. Lab., Univ. of Washington, Seattle, WA 98105)

The main goals of the APL program in SAX99 were to measure and improve our ability to model acoustic propagation within, high-frequency backscattering from, and penetration into sand sediments. To prepare for these measurements, new equipment and experimental procedures were developed. For the penetration studies, simulations were used extensively to guide the experiment design in order to ensure that the measurements would be useful for addressing our goals. Illustrations will be given of how simulations were used to support the experiment design. The APL experimental equipment used in SAX99 will be described, and the experimental procedures will be presented. Finally, the resulting data set will be summarized. [Work supported by ONR.]

$8: 45$

2aUW4. Acoustic penetration at subcritical grazing angles: Measurements and comparison with simulations based on perturbation theory. Darrell R. Jackson, Kevin L. Williams, Dajun Tang, and Eric I. Thorsos (Appl. Phys. Lab., Univ. of Washington, Seattle, WA 98105)

As part of the SAX99 experiment, a buried hydrophone array was deployed together with a movable tower with attached sources covering the frequency range $11-50 \mathrm{kHz}$. This system was used to examine subcritical penetration into the sediment. For incident grazing angles below the critical angle, scattering dominates the penetrating field. Comparisons with simulations based on perturbation theory show that the penetration is predominately the result of diffraction by the low-amplitude ripple field prevalent at the SAX99 site. Simulations predict a cutoff effect as a function of frequency and grazing angle that is found in the data, and predict changes in penetration as a function of ripple field amplitude that are consistent with those observed. [Work supported by ONR.]

9:00

2aUW5. Refraction and scattering in sandy sediments. Nicholas $P$. Chotiros, D. Eric Smith, and James N. Piper (Appl. Res. Labs., Univ. of Texas, P.O. Box 8029, Austin, TX 78713-8029, chotiros@arlut.utexas.edu)

Signals collected from a buried acoustic receiving array in a sandy sediment off the west coast of Florida, as part of the Sediment Acoustics Experiment (SAX99), were analyzed to determine the penetration processes. The two main candidate processes are refraction and scattering. Wave front coherence and direction of arrival, as well as sound speed measurements, provide important clues concerning the processes involved. The sound was projected by a wideband sound source carried on a re- 
motely operated vehicle (ROV) in the band 10 to $100 \mathrm{kHz}$. Coherent and incoherent processing methods were explored. [Work supported by the Office of Naval Research, Ocean Acoustics.]

\section{$9: 15$}

2aUW6. Subcritical acoustic scattering across a rough fluid-solid interface-laboratory experiment and perturbation theory model. Garfield R. Mellema (Defence Res. Establishment Atlantic, P.O. Box 1012, Dartmouth, NS B2Y 3Z7, Canada), Terry E. Ewart, and Kevin L. Williams (Univ. of Washington, Seattle, WA 98105)

Several mechanisms have been proposed by which an incident acoustic wave could be coupled into the ocean floor at angles below the compressional critical angle. Recent observations of acoustic transmissions from water into sediment with arrival times and amplitudes inconsistent with the refractive compressional path have been interpreted as the excitation of a Biot slow wave in the sediment. Another hypothesis attributes the observed signals to scattering at the rough water-sediment interface. A third entails scattering of evanescent waves by volume inhomogeneities in the sediment. The existence of multiple hypotheses, each of which could account for the received energy, invited further investigation. A series of well-controlled laboratory measurements were made and compared with the results of a first-order perturbation theory model in order to evaluate the accuracy of the rough interface hypothesis. The experimental measurements of transmission through adjacent flat and rough interfaces show good agreement with the results of the numerical model, giving clear evidence of subcritical penetration in an environment incompatible with the requirements of the Biot slow wave and volume scattering hypotheses.

\section{9:30-9:45 Break}

\section{$9: 45$}

2aUW7. High-frequency measurements of absorption and dispersion in a sandy sediment. Darrell R. Jackson, Kevin L. Williams, Dajun Tang, and Eric I. Thorsos (Appl. Phys. Lab., Univ. of Washington, Seattle, WA 98105)

As part of the SAX99 experiment, a buried hydrophone array was deployed together with a movable tower with attached sources covering the frequency range $11-50 \mathrm{kHz}$. With the tower placed to provide incident grazing angles well above the critical angle, this system was used to obtain data from which sediment sound speed and absorption were determined. The sound-speed data exhibit significant dispersion, while the absorption data show an approximate linear frequency dependence. When these data are combined with data at other frequencies from the same site, the dispersion and absorption are found to be consistent with causality and with the Biot model [Work supported by ONR.]

\section{0:00}

2aUW8. Velocity dispersion in water-saturated granular sediment. Robert D. Stoll (Lamont-Doherty Earth Observatory of Columbia Univ., Palisades, NY 10964, stoll@1deo.columbia.edu)

When the Biot theory is applied to problems in sediment acoustics, significant velocity dispersion is predicted over a specific frequency range that depends on the pore-fluid mobility (permeability). This dispersion, and the associated nonlinear variation of attenuation with frequency, affects the reflection and transmission characteristics of the water-sediment interface, particularly at low grazing angles. Low-frequency velocity measurements taken during the ONR SAX99 experiments, when considered together with the high-frequency data, verify the existence of this dispersion. This result has important implications in the choice of critical angle and the interpretation and extrapolation of velocity and attenuation data taken at high frequencies. [Work supported by ONR, Code 321OA.]

\section{0:15}

2aUW9. Acoustic wave propagation in granular media. Masao Kimura (Dept. of Geo-Environ. Technol., Tokai Univ., 3-20-1 Orido, Shimizu, Shizuoka, 424-8610 Japan, mkimura@scc.u-tokai.ac.jp) and Nicholas P. Chotiros (Univ. of Texas, Austin, TX 78712)

The frame bulk and shear moduli are important in analyzing the acoustic wave propagation in marine sediments. The frame bulk and shear moduli of marine sediment are related to the longitudinal and shear wave velocities in dry marine sediments. The measured results of the longitudinal wave velocities in dry glass beads with four different grain sizes, but with almost the same porosity have been reported. The measured results showed that the longitudinal wave velocity largely increases, as the grain size increases. This result could not be explained using the classical contact theory, such as the Hertz-Mindlin or Digby model. In this study, we use the longitudinal wave velocities of dry glass beads in air and in vacuum to investigate the effects of the air, as the pore fluid. The measured results show that the longitudinal wave velocities in vacuum are smaller than that in air, and the velocities in vacuum become almost constant, even though the grain sizes are different.

\section{0:30}

2aUW10. Sediment acoustic backscattering during SAX99: Measurements and models. Kevin L. Williams, Darrell R. Jackson, Dajun Tang, and Eric I. Thorsos (Appl. Phys. Lab., Univ. of Washington, Seattle, WA 98105)

During the SAX99 experiment, acoustic backscattering measurements were made at frequencies from 20 to $300 \mathrm{kHz}$ as a function of grazing angle. The results from these acoustic measurements will be presented and compared with backscattering models that use the environmental measurements of other SAX99 researchers as input. In the $20-50-\mathrm{kHz}$ range these comparisons indicate that surface roughness plays a dominant role in acoustic backscattering with a very distinctive reduction in backscattering at grazing angles above the critical angle of the sediment. Above $50 \mathrm{kHz}$ this critical angle feature is less evident. Possible reasons for this change with frequency will be discussed. The backscattering models used here were originally developed for frequencies from 10 to $100 \mathrm{kHz}$. SAX99 data give some indication that further modeling is needed above $100 \mathrm{kHz}$. [Work supported by ONR.]

\section{$10: 45$}

2aUW11. Biological and hydrodynamic control of seafloor roughness: Implications to high-frequency acoustic scattering. Michael D. Richardson, Kevin B. Briggs (Marine Geosciences Div., Naval Res. Lab., Stennis Space Center, MS 39529-5004), and Kevin L. Williams (Univ. of Washington, Seattle, WA 98105-6698)

Biological and hydrodynamic processes can both create and destroy seafloor microtopography. As part of the SAX99 experiments, natural and artificial temporal changes in seafloor roughness were monitored acoustically and quantified using bottom stereo photographs. Feeding activities of benthic megafauna and fish destroyed large-scale roughness features generated by ocean surface gravity waves within a period of weeks to months; whereas, fine-scale roughness created by raking the seafloor decayed to background levels within $24 \mathrm{~h}$. The effects of fine-scale roughness increased acoustic scattering centered at one-half the acoustic wavelength (a Bragg wavelength of $2 \mathrm{~cm}$ ) by $12-18 \mathrm{~dB}$ in artificial manipulations of the bottom. These changes were restricted to roughness that was oriented predominantly orthogonal to the incident acoustic waves. Alternatively, 
seafloor roughness generated by ocean surface gravity waves had wavelengths of $50-100 \mathrm{~cm}$ and wave heights of $10-15 \mathrm{~cm}$. These predictable large-scale roughness features should, by analogy, dramatically increase scattering at lower acoustic frequencies (near $1-2 \mathrm{kHz}$ ) and decay within weeks to months after storm events. [Work supported by ONR.]

\section{1:00}

2aUW12. High-frequency, low-grazing-angle backscattering from a sandy bottom. Charles F. Greenlaw, D. Van Holliday, and Duncan E. McGehee (Bae Systems, 4669 Murphy Canyon Rd., San Diego, CA 92123)

Backscattering measurements from a sandy bottom off East Destin, FL were obtained using a multi-frequency echosounder attached to a pan/tilt head on a bottom-mounted tower during the Sediment Acoustics Experiment, SAX99. Usable data were obtained over the frequency range from 265-1850 kHz, spanning the transition from roughness-controlled to grain-size-controlled scattering, at grazing angles around 15. Field results will be discussed and compared to laboratory measurements. [Work supported by ONR Code 322 BC.]
11:15

2aUW13. The small slope approximation for acoustic scattering from a rough interface between a fluid and a fluid-saturated porous solid. Cristina Fetche, Shira L. Broschat (School of Elec. Eng. and Computer Sci., Washington State Univ., P.O. Box 642752, Pullman, WA 99164-2752), and Taiqian Yang (Central Washington Univ., Puyallup, WA 98374)

The small slope approximation, introduced by A. G. Voronovich in the 1980s, gives a systematic expansion in terms of a generalized surface slope. Previous numerical studies indicate that the lowest-order term provides a reasonably accurate approximation for a number of different problems of practical importance. In this work we consider the small slope approximation for acoustic scattering from a rough interface between a fluid and a fluid-saturated porous solid in the context of Biot theory. Biot theory is of continued interest in the study of sediment acoustics; it predicts a slow compressional wave in the fluid-filled porous medium in addition to the conventional compressional and shear waves found in an elastic solid. The lowest-order small slope approximation is derived for Biot theory, and an expression for the bistatic scattering cross section is presented for plane-wave scattering from a randomly rough interface between a fluid and a fluid-saturated porous solid. Numerical results for the scattering strength are discussed for both the small slope and perturbation methods. [Work supported by ONR.]

\title{
Session 2pAA
}

\section{Architectural Acoustics: Building Renovation and Sound Scattering and Absorption}

\author{
Courtney B. Burroughs, Chair \\ Applied Research Laboratory, Pennsylvania State University, P.O. Box 30, State College, Pennsylvania 16804
}

\section{Contributed Papers}

$1: 30$

2pAA1. Acoustics of a remodeled historic building. Ballard W. George (Environtech Consultants, 1367 Bobolink Circle, Sunnyvale, CA 94087)

An acoustical study was performed for a historic San Francisco industrial building being remodeled for office, restaurant, and exhibit use. Challenges involving restoration ground rules included open beam construction with no use of ceilings. Flooring is proposed to be hardwood. Damped plywood was recommended to be used in flooring assemblies to enhance airborne and impact sound insulation performance. The building is across the street from the newly opened Pac tel baseball park for the San Francisco Giants. Accordingly, exterior noise sources included both automobile and bus traffic and the various sounds associated with baseball games. Sound measurements were taken at the 3 Com Park on Candlestick Point as an aid in predicting the sound levels associated with the new park. The acoustical study addressed the restaurants and exhibit spaces on the lower floors, the separating flooring assemblies between each level, and the sound "delivered" by ducting, and otherwise transmitted, to the future tenant spaces. Comments are given on the "soundscape" considerations associated with the various spaces of the lower floors of the building.

\section{$1: 45$}

2pAA2. Room boundary absorption coefficient measurements in a small room. Jerald R. Hyde (JRH Acoustics, Box 55, St. Helena, CA 94574)

During the renovation of an 1890s Victorian building, the author's high ceiling office became entirely empty, the rectilinear boundaries consisting of wood, plaster, and glass surfaces. With a volume of $86 \mathrm{~m}^{3}$ (3030 $\mathrm{ft}^{3}$ ) the empty room had reverberation times (RT) ranging from $1 \mathrm{sec}$ at low frequencies to over 2-1/2 sec at high frequencies. Boundary absorption coefficients were derived from RT data for the empty room's surfaces with the greatest absorption occurring in the $125-\mathrm{Hz}$ octave band. Two different (weight) carpets of approximately $8.5 \mathrm{~m}^{2}\left(92 \mathrm{ft}^{2}\right)$ were then added to the floor and their absorption values calculated. Absorption coefficient results will be presented along with a study of the derived coefficient values of each of the component surfaces. Similar "boundary only" results from an "empty" 500-seat theater will also be shown.

2:00

2pAA3. Scale-model MLS measurements of edge diffraction. Rendell R. Torres (Chalmers Rm. Acoust. Group, Chalmers Univ. of Tech., SE-412 96 Gothenburg, Sweden) and Michael Vorländer (Tech. Univ. of Aachen, Aachen, Germany)

To better understand edge diffraction in room acoustics, scattering is measured from 1:10 scale models of reflector arrays and a stage house. The source is an 11-cm dodecahedron with tweeter elements having an effective frequency range of 600-6000 Hz. Maximum-length sequences (MLS) are used, which have various advantages over spark sources for scale models. The main three reflector arrays have approximately the same equivalent area: an array of five long rectangular panels, a 7-by-5 array of smaller square panels, and a single solid panel. Measurements are taken along a line under the reflector arrays and repeated for different array sizes and coverage. The stage-house measurements include a stepped reflector array and receiver positions shadowed from the receiver. Results compare well with previous work [R. W. Leonard et al., J. Acoust. Soc. Am. 36, 
2328-2333 (1964)], and some practical rules of thumb are discussed. [Work performed as guest of the Inst. fuer Techn. Akustik, RWTHAachen, Germany; support provided by the Axson Johnson Foundation, Sweden.]

\section{2:15}

2pAA4. Pressure-amplitude reflection coefficients of active impedance tube terminations and their relationships to energy-related quantities of adjacent sound fields. Timothy W. Leishman (Dept. of Phys. and Astron., Brigham Young Univ., Provo, UT 84602, tim_leishman@byu.edu)

Active boundary surfaces intended to control reverberation or other characteristics of enclosed sound fields have often been investigated using impedance tubes. Unfortunately, the significance of pressure-amplitude reflection coefficients produced by these surfaces and their relationships to acoustic energy quantities in enclosed spaces has remained obscure. This paper accordingly presents a straightforward analysis of an actively terminated impedance tube to provide needed clarification. It demonstrates that increases in reflection coefficient moduli at the termination do not necessarily produce proportional increases in total enclosed energy in the tube or energy flux toward the enclosed field. Very large reflection coefficients (i.e., with moduli much greater than unity) produce both total enclosed acoustic energies and vector magnitudes of net active intensity approaching those present under an anechoic boundary condition. Large relative changes in total enclosed energy occur only when active termination reflection coefficient moduli roughly approach unity. Energy-related effects of actively produced reflection coefficients are generally frequencydependent functions of distinct enclosed spaces and acoustic fields generated by primary sources in these spaces. Hence, correlations between reflection coefficient moduli and energy quantities of adjacent sound fields are not necessarily simple or consistent. They depend upon important physical properties of acoustic spaces, sources, and source arrangements.

\section{2:30}

2pAA5. Normal-incidence sound absorption measurement of a perforated panel using a sound-intensity technique: Theoretical versus experimental. Mohamad N. Dimon, Tharek A. Rahman, Ahmad K. Said (Elec. Eng. Faculty, UTM, 81310, Skudai, Johor, Malaysia), M. N. Ibrahim (UTM, 81310, Skudai, Johor, Malaysia), and M. Y. Jaafar (MARDI, Serdang, Selangor, Malaysia)

A direct-piercing carved-wood panel (DPCWP) is a form of wood carving that allows air to pass through the panel easily. In Malaysia, DPCWPs are available with various perforation ratios, patterns, and elements, and are typically used as part of a wall partition for an enclosed room. It helps in providing natural ventilation, besides it is believed to be able to act as a sound absorber. Circular perforated panels are the simplest form of DPCWP available. It was found out that normal-incidence soundabsorption performance of perforated panels can be measured reliably using a sound-intensity measurement technique. In this paper, normalincidence sound-absorption measurement of a perfortated panel using fixed-point scanning will be discussed. Close correlations were obtained from $250 \mathrm{~Hz}$ to $4 \mathrm{kHz}$ between theoretical and measured sound absorption. Factors contributing toward sound intensity measurement reliability, primarily through its sound quality indicators such as $F 1, F 2, F 3$, and $F 4$, and the effect of measurement distance will be thoroughly discussed. This finding suggests that the sound intensity measurement technique can be used to measure normal-incidence sound-absorption performance of DPCWP reliably.

\section{2:45}

2pAA6. Acoustic properties of a triple-leaf membrane. Kimihiro Sakagami, Masayuki Morimoto (Environ. Acoust. Lab., Kobe Univ., Nada, Kobe, 657-8501 Japan, saka@kobe-u.ac.jp), and Masakazu Kiyama (Nippon Sheet Glass Environment Amenity Co., Ltd., Osaka, 541-0045 Japan)

Acoustic properties of a triple-leaf membrane, consisting of two impermeable and one permeable leaves, are theoretically analyzed under a simple condition in which a plane wave is incident normally. The tripleleaf membrane shows different acoustic properties according to where the permeable leaf is placed. When the permeable leaf is placed in the cavity between two impermeable leaves, its absorptivity shows two mass-airmass resonance peaks, one by the first and second leaves, and the other by the first and third leaves, provided that the permeable leaf is of sufficient flow resistance and mass. These peaks can be merged to produce one larger peak so that the structure can offer higher absorptivity over a wider frequency range, when the masses of leaves and the cavity depths are adequately adjusted. A parametric study shows that the sound absorption and insulation performances of a triple-leaf membrane of this type are better than those of a double leaf with an air cavity only, though a double leaf with an absorbent in its cavity is still the most efficient. A method to optimize the system parameters to obtain maximum absorptivity is also suggested. Another arrangement, with the permeable leaf as the first leaf, is also explored. 


\title{
Session 2pABa
}

\section{Animal Bioacoustics: Distinguished Lecture-Ultrasonic Eyeglasses for the Blind}

\author{
Whitlow W. L. Au, Chair \\ Hawaii Institute of Marine Biology, P.O. Box 1106, Kailua, Hawaii 96734 \\ Chair's Introduction-1:00
}

1:05

2pABa1. Ultrasonic eyeglasses for the blind. Leslie Kay (Spatial Sensing Lab., Sonicvision Ltd., P.O. Box 124, Russell, New Zealand)

There is an obvious need for blind persons to perceive their environment in the greatest detail possible. The bat has demonstrated the effective use of ultrasonic waves for its own purpose of perceiving its environment. Man now has his own air-sonar technology, in the form of eyeglasses mounted on the head, so as to perceive his environment. It may well be the best that can be achieved for helping blind persons by using their auditory channels to the cortex for spatial imaging and cognition of object space. This address describes the octave band CTFM air sonar with auditory coupling to the brain. The method of testing its physical performance is related to the human auditory perception of object space experienced by users. FFT analysis shows a distal resolution of approximately two wavelengths. However, behavioral analysis of a user performance suggests a greater neural "resolution" of object space is available. This resolution is demonstrated by the natural real-time perception of object-shape characteristics. The "infinity" of sonar object "signatures" are seemingly absorbed by the listener's neural system and eventually learned. The small nuances in the real-time change in sound structure as an object pose is altered are invariant. This requires an enormous memory.

\section{Session 2pABb}

\section{Animal Bioacoustics and Noise: Standards and Animals: Where Are We?}

\author{
Ann E. Bowles, Chair
}

Hubbs-Sea World Research Institute, 2595 Ingraham Street, San Diego, California 92109

Invited Papers

2pABb1. The development of human standards to limit noise-induced hearing loss: A good model for animals? Ann E. Bowles (Hubbs-Sea World Res. Inst., 2595 Ingraham St., San Diego, CA) and Daniel L. Johnson (Interactive Acoustics, Provo, UT)

Over the past 50 years, the ASA and other organizations have promulgated standards to protect human hearing. We are evaluating these standards as a model for similar standards for animals. First, we must eliminate noise at frequencies that could have little effect on hearing. In the case of humans, A-weighting has been selected for most applications. The choice was determined partly by convenience (readily available circuitry, computationally simple calculations for weighted values) and partly by its robustness in fitting empirical data. Second, allowable exposures must take into account exposure time. For humans, the time-intensity tradeoff is calculated on an equal-energy basis that presumes underlying linear functions (the basis of the 3-, 4-, and 5-dB exchange values used by various organizations), again to simplify computation. For high-amplitude, brief transients (impulse noise), which produce nonlinear auditory responses, an absolute upper limit of exposure is used. This scheme has proved adequate to protect crucial human hearing capabilities (mainly the understanding of speech), but may not be conservative enough for animals, which use their hearing in self-defense, for navigation, etc. It is interesting to note that schemes for animals can be developed without the computational simplicity that historically drove many decisions in the human case. 
2pABb2. A preliminary study of loudness at frequencies of 5 to $120 \mathrm{kHz}$ based on whistle response time (RT) in a dolphin. Sam Ridgway and Donald Carder (Marine Mammal Prog., Space and Naval Warfare Systems Ctr., 49620 Beluga Rd., San Diego, CA 92152-6266)

Stebbins (1966) showed that auditory RT of monkeys could be used to measure loudness. Such studies have been replicated in other animals and humans. Although Ridgway et al. (1991) reported that dolphin auditory RT varied with stimulus intensity, curves showing variation in RT with frequency and amplitude were not presented. Choice RT was measured in a female bottlenose dolphin responding to $300 \mathrm{msec}$ bursts of clicks (30 clicks at $10 \mathrm{~ms}$ ISI) with a burst of pulses, and to $300 \mathrm{msec}$ tones with a whistle. Responses were received with a hydrophone (B\&K 8103), digitized, and stored on disk. The dolphin responded correctly (whistled to the tone, pulsed to the pulses) on more than $98 \%$ of presentations. Here we only consider tone presentations. Frequencies, presented randomly, were $5,10,20,40,60,80,100$, and $120 \mathrm{kHz}$ and ranged from 85 to $135 \mathrm{~dB}$ re 1 Pa rms. At $10 \mathrm{kHz}$, median RT ranged from $240 \mathrm{msec}$ at $135 \mathrm{~dB}$ to $410 \mathrm{msec}$ at $85 \mathrm{~dB}$. At $100 \mathrm{kHz}$, median RT ranged from $260 \mathrm{msec}$ at $135 \mathrm{~dB}$ to $330 \mathrm{msec}$ at $85 \mathrm{~dB}$. Studies must be done over a larger range of amplitude and frequency and with precise control of background noise.

3:00

2pABb3. Statistical requirements to demonstrate subtle impacts of noise on passerine bird populations. Don Hunsaker, II (Hubbs Sea World Res. Inst., 2595 Ingraham St., San Diego, CA 92109)

We are conducting a study on the effects of noise on California gnatcather (CAGN) and least Bell's vireo (LBV) populations at military installations in San Diego, CA. In order to improve the statistical power to be able to accurately measure subtle effects of noise on the birds, we have had to expand the size of the study considerably. We have mapped noise levels and reproductive success in the study area. This provides an estimation of the noise covariate at all the sampled nests and enables us to characterize the magnitude of effects of noise on reproductive success by estimating the strength of correlation between noise and nesting success endpoints. Our analysis determined that 60 nesting pairs would provide data to detect a $20 \%$ difference at the $5 \%$ significance level with $80 \%$ power. This ability to measure only gross impacts was unacceptable to us. The design now includes 5 years to sample 300 nests of CAGN and 800-1000 nests of LBV for us to achieve the power to assess these subtle effects at the 5\% level.

3:20

2pABb4. Assessing ocean noise: A conservationist approach. Joel R. Reynolds and Michael D. Jasny (Natural Resources Defense Council, 6310 San Vicente Blvd., Ste. 250, Los Angeles, CA 90048)

The regulation of ocean noise is an evolving process that involves representatives of the environmental community as well as scientists and regulators. This paper describes a three-stage procedure, based on the precautionary principle, that is currently being used by environmentalists to identify acoustic projects with potentially significant biological impacts: (1) a threshold stage of review, in which factors such as the ecological significance of the operation area, the intensity, frequency, and duration of the source, the mitigation offered by project applicants, and the cumulative and precedential impacts of the project are considered; (2) a period of consultation with experts in the field; and (3) an assessment of the project according to the substantive, procedural, and evidentiary standards set forth by applicable statutes and regulations, including the National Environmental Policy Act, Marine Mammal Protection Act, and Endangered Species Act. In the third stage, projects are evaluated according to the integrity of their alternatives analysis, especially with regard to location and season; the consistency of their environmental assessment with the best available science; the significance of their reasonably anticipated cumulative impacts; their objectivity in identifying data gaps; and their commitment through mitigation to have the least practicable impact on the marine environment.

\section{Contributed Papers}

3:40

2pABb5. Masked temporary threshold shift (MTTS) in odontocetes after exposure to single underwater impulses from a seismic watergun. James J. Finneran (Marine Mammal Prog., Space and Naval Warfare Systems Ctr., 49620 Beluga Rd., San Diego, CA 92152-6266), Carolyn E. Schlundt, Randall Dear (Sci. Applications Intl. Corp., San Diego, CA 92110), Donald A. Carder, and Sam H. Ridgway (Space and Naval Warfare Systems Ctr., San Diego, CA 92152-6266)

A behavioral response paradigm was used to measure masked underwater hearing thresholds in a bottlenose dolphin (Tursiops truncatus) and white whale (Delphinapterus leucas) before and after exposure to single underwater impulses produced by a seismic watergun. Hearing thresholds were measured at $0.4,4$, and $30 \mathrm{kHz}$ using a vocal response technique and a modified version of the Method of Free Response. A pre-exposure threshold was generally measured $34 \mathrm{~min}$ before presentation of the impulse. Post-exposure threshold measurements began within 2 min of exposure to the impulse and continued for approximately 20 min following exposure, in order to track recovery. A masked temporary threshold shift (MTTS) was defined as a 6-dB or larger increase in threshold compared to the corresponding pre-exposure measurement. The MTTS test paradigm will be discussed and preliminary MTTS and behavioral response data presented and compared to existing TTS data for odontocetes. [Work supported by ONR.]
3:55

2pABb6. Underwater temporary threshold shifts in pinnipeds: Tradeoffs between noise intensity and duration. Ronald Schusterman, David Kastak, Brandon Southall, and Colleen Kastak (Long Marine Lab., Univ. of California, Santa Cruz, 100 Shaffer Rd., Santa Cruz, CA 95060, rjschust@cats.ucsc.edu)

Temporary threshold shifts (TTSs) were obtained in one female California sea lion (Zalophus californianus), one male harbor seal (Phoca vitulina), and one female northern elephant seal (Mirounga angustirostris). Prior to noise exposure, base-line hearing thresholds were obtained at 2500 or $3530 \mathrm{~Hz}$ using behavioral techniques. During noise exposure, the subjects were stationed in an underwater noise field for either 20-25 or 50 total minutes. The noise field consisted of octave-band noise centered at $2500 \mathrm{~Hz}$ with exposure levels of either 65,80 , or $95 \mathrm{~dB}$ above the mean base-line thresholds [i.e., sensation level (SL)]. Postexposure thresholds were obtained immediately following noise exposure. The same testing and diving patterns were used in the absence of noise to control for the effects of diving. Hearing losses ranged from 0 to $28 \mathrm{~dB}$, and there was substantial inter- and intraspecific variability in the amount of hearing loss following exposure to similar noise regimes. Recovery was also variable, ranging from minutes to hours, with complete recovery always occurring within 24 hours. We found threshold shifts of $5 \mathrm{~dB}$ at $65 \mathrm{~dB}$ SL and only 
marginal increases in TTS with increasing noise intensity. However, increasing noise duration with constant noise exposure levels yielded greater shifts.

\section{4:10}

2pABb7. A comparison of behavioral and auditory brainstem response methods for examining hearing sensitivities in the harbor seal (Phoca vitulina). Lawrence F. Wolski, Rindy C. Anderson, and Ann E. Bowles (Hubbs-SeaWorld Res. Inst., 2595 Ingraham St., San Diego, CA 92109)

Auditory brainstem response (ABR) and behavioral methods (method of constant limits and staircase method) were used to generate in-air audiograms for an adult female harbor seal to examine relative sensitivities obtained using the two methods. Behavioral thresholds ranged from
$0.250-30 \mathrm{kHz}$ and indicated best sensitivity from $6-12 \mathrm{kHz}(\sim 12 \mathrm{~dB}$ re: $20 \mu \mathrm{Pa}$ ). The staircase method was more sensitive than the method of constant limits, resulting in thresholds averaging $10 \mathrm{~dB}$ lower. ABR thresholds were recorded at 2, 4, 8, 16, and $22 \mathrm{kHz}$ and showed a similar range of best sensitivity $(8-16 \mathrm{kHz})$. ABR and behavioral thresholds differed by an average of $5 \mathrm{~dB}$; ABR thresholds averaged $3.8 \mathrm{~dB}$ higher than behavioral thresholds from $2-8 \mathrm{kHz}$, but were 12 and $3 \mathrm{~dB}$ lower at 16 and $22 \mathrm{kHz}$, respectively. The lower ABR thresholds at higher frequencies may reflect the behavior of the seal during ABR testing as well as broadband characteristics of ABR test stimuli. These results agree with comparisons of $\mathrm{ABR}$ and behavioral methods performed in other recent studies and indicate that auditory evoked potential methods represent an accurate alternative to behavioral methods for estimating hearing range and sensitivity in wild animals. [Work supported by NASA: NAS1-20101.]

$4: 25-5: 10$

Panel Discussion

TUESDAY AFTERNOON, 5 DECEMBER 2000

PACIFIC SALON D, 1:00 TO 5:50 P.M.

Session 2pBB

\title{
Biomedical Ultrasound/Bioresponse to Vibration, Physical Acoustics and Acoustical Oceanography: Detection and Characterization of Bubbles, Acoustic Cavitation, and Associated Physical Effects II
}

\author{
Ronald A. Roy, Cochair \\ Department of Aerospace and Mechanical Engineering, Boston University, 110 Cummington Street, \\ Boston, Massachusetts 02215 \\ Timothy G. Leighton, Cochair \\ Institute of Sound and Vibration Research, University of Southampton, Highfield, Southampton SO17 1BJ, United Kingdom \\ Chair's Introduction-1:00 \\ Invited Papers
}

1:05

2pBB1. Characterization of measures of reference acoustic cavitation (COMORAC). Timothy G. Leighton (Inst. of Sound and Vib. Res., Univ. of Southampton, Highfield, Southampton SO17 1BJ, UK)

This paper describes a coordinated experiment involving a number of UK centers, with the aim of comparing a range of systems which measure cavitational activity. Sensors have been designed at a number of universities to produce quantitative outputs based on sonochemical (Bath and Southampton), calorimetric (Coventry), electrochemical and luminescent (Southampton) measures. In addition, acoustic sensors have been produced (Guys and St. Thomas Hospital Trust, and the National Physical Laboratory). These are all deployed to monitor controlled cavitation events, ranging from single bubble phenomena to a reference cavitation bath (produced by NPL). Design of these cavitation systems has involved not only the characterization of the sound field and the vessel, but also the specification of some reference cavitation liquid (NPL and Southampton). Comparison of the resulting measures of activity allows the feasibility of a scale for cavitation to be investigated.

$1: 30$

2pBB2. Optical Mie scattering to observe single cavitation bubble dynamics. R. Glynn Holt (Dept. of Aerosp. and Mech. Eng., Boston Univ., 110 Cummington St., Boston, MA 02215, rgholt@bu.edu)

It is often advantageous to know precisely the instantaneous response of bubbles to an external acoustic field. One technique to enable such observations consists of detecting the time-resolved optical scattering of an incident laser beam from a bubble. For visible laser light and bubble radii on the order of 1-100 microns, reflection, refraction, internal interference, and diffraction must all be taken into account, and the scattering so described is named after Gustav Mie. Physical principles and the mathematical development will be discussed. Several specific applications will be presented and the results discussed. Finally, a brief list of "do's and don'ts" for the potential user will be discussed. 
2pBB3. When is a bubble a bubble?: The subtleties of cavitation threshold detection at MHz frequencies. Ronald A. Roy (Dept. of Aerosp. and Mech. Eng., Boston Univ., Boston, MA 02215)

Detecting the onset of cavitation induced by short-pulse ultrasound at $\mathrm{MHz}$ frequencies is tricky business, for one must be positioned to observe the genesis and evolution of a single micron-sized bubble lasting as short as one acoustic cycle. The observation is rarely accomplished directly. Rather, investigators often rely on indirect evidence of cavitation activity manifested by physical effects such as noise (a.k.a. passive cavitation detection), light emission, and mechanical damage. These techniques are noise limited, and investigators frequently measure a threshold for "detectable" cavitation. Another approach is to actively probe the medium with a high-frequency acoustic beam, and watch for anomalous backscatter associated with bubble activity. This technique, often termed active cavitation detection, can be quite sensitive, which is part of the problem; transient disturbances in the medium can be mistaken for bona fide cavitation activity. Such errors are often associated with, say, the motion of solid particles in a test liquid that is put into motion by acoustic radiation forces or entrained by acoustic streaming. In all cases, the ability to detect and monitor minute, spurious cavitation activity is limited by noise, clutter, or reverberation. These issues are discussed, examples given, and potential solutions proposed. [Work supported by DARPA.]

2pBB4. Acoustic spectrum and sonoluminescence as indicators of cell membrane disruption by ultrasound. Mark R. Prausnitz, Hector R. Guzman, and Stephen Cochran (Schools of Chemical and Biomed. Eng., Georgia Tech, Atlanta, GA 30332-0100)

Ultrasound has been shown to reversibly disrupt cell membranes and thereby drive drugs and genes into cells by a mechanism believed to involve cavitation. We have measured the number of molecules delivered into cells and levels of cell viability following exposure to a broad range of ultrasound conditions. Many cellular responses are possible, including uptake of up to millions of molecules per cell and cell viability ranging from 0 to $100 \%$. Because these effects are believed to occur due to acoustic cavitation, we measured features of the acoustic spectrum known to be associated with cavitation and measured sonoluminescence light output during ultrasound exposures. We found that these measures of cavitation correlate with both the number of molecules taken up by cells and levels of cell viability. This observation strengthens the hypothesis that ultrasound's bioeffects are mediated by cavitation and suggests a noninvasive means to assess these bioeffects which could be used as part of a real-time feedback loop. [Work supported by the NSF, NIH, and Whitaker Foundation.]

\section{2:45}

2pBB5. Control of inertial cavitation in shockwave lithotripsy. Andrew J. Coleman (Med. Phys. Dept., Guy's and St. Thomas' Hospital, London SE1 7EH, UK, andrew.coleman@gstt.sthames.nhs.uk) and Mark D. Cahill (Univ. of Bath, Bath BA2 7AY, UK)

The role of inertial cavitation in stone comminution and tissue damage during extracorporeal shock-wave lithotripsy (ESWL) became appreciated several years after the successful introduction of ESWL into routine clinical use in the early 1980s. It is only relatively recently, however, that attempts have been made to enhance or suppress the cavitation field in lithotripsy by adapting the acoustic field generated by the shock-wave source. The single-cycle acoustic pulses used in commercial ESWL generate a cavitation field in which the bubbles may remain in an expanded phase for many times the pulse duration. Some control of cavitation can be gained by timing an additional pulse from a second source to coincide, for example, with the bubbles expanded phase [M. R. Bailey, Tech. Rep. No. ARL-TR-97-1, Univ. of Texas, Austin (1997)]. This can generate an earlier and more violent bubble collapse. A single source generating multiple-cycle pulses can, similarly, provide a means of controlling cavitation activity in lithotripsy. This approach has been examined using an electromagnetic-type shock-wave source to generate high-amplitude, multicycle pulses in water. By tailoring the pressure waveform specifically to control cavitation, it may be possible to refine medical ultrasound applications, such as ESWL, where cavitation plays a role.

3:10

2pBB6. Characterization of ultrasound contrast agent oscillations. Katherine Ferrara, Karen Morgan, James Chomas, and John Allen (Div. of Biomed. Eng., Univ. of California, Davis, One Shields Ave., Davis, CA 95616, kwferrar@ucdavis.edu)

Ultrasound contrast agents consist of a gas bubble surrounded by a shell, and are used in studies of tissue perfusion. It is currently difficult to differentiate contrast agent echoes from nonlinear components of tissue echoes. A goal of this research is to improve the understanding of individual contrast agent oscillations and the resulting received echoes. This, in turn, can lead to more sophisticated bubble detection techniques by designing pulsing schemes to elicit a specific bubble response. In this study, experimental and theoretical tools are uniquely combined to aid in the understanding of a bubble's response during insonation. A model is used for comparison, which is based on the equation developed by Rayleigh for an unencapsulated bubble, with additional terms to include the effects of shell elasticity and viscosity. The resulting radius-time curves are validated by direct comparison with the experimental results from a high-speed optical system capable of 100 million frames/s. One resulting signal processing strategy involves the transmission of a train of wide-band pulses with alternating phase which yields echoes with significant spectral differences, quantified by calculating the mean frequency of each echo. A new bubble detection technique is proposed based on these differences in mean frequency.

3:35-3:50 Break 


\section{Contributed Papers}

2pBB7. Nonlinear scatter from the ultrasound contrast agent Sonazoid: Theory and numerical simulations. Lars Hoff (Dept. of Telecommunications, Norwegian Univ. of Sci. and Technol., N-7491 Trondheim, Norway)

A model for the contrast agent Sonazoid is presented. The model is based on a modified Rayleigh-Plesset equation, with the shell added as an incompressible viscoelastic solid. Different models for the shell's nonlinear stress-strain relationship are discussed. Shell elasticity and viscosity were estimated from experiments, and the model was used to simulate bubble radius and scattered sound. Pulse-inversion imaging was simulated. The amplitude was increased from 20 to $500 \mathrm{kPa}$, giving scattered pulses that changed from inverted to time-shifted versions of each other. This effect was best seen in the cross correlation of the responses. The nonlinear response was strongest for frequencies below bubble resonance. Compared to unshelled bubbles, Sonazoid required higher drive amplitudes to respond nonlinearly. The Sonazoid bubbles responded similarly to unshelled bubbles of smaller diameter. A subharmonic response was found in a limited frequency band and only for diameters above $3 \mu \mathrm{m}$. An amplitude threshold was found around $500 \mathrm{kPa}$, depending on diameter and frequency. This threshold is higher than for unshelled bubbles. A chaotic oscillation pattern was found for some diameter-frequency combinations, in agreement with published results. For some situations where the $\mathrm{cw}$ results predicted a subharmonic mode, this mode was not obtained from finite-length pulses.

\section{4:05}

2pBB8. Interaction of shock waves with cavitation bubble: Application of Ho:YAG pulse laser irradiation for cerebral thrombolysis. S. H. R. Hosseini (Shock Wave Res. Ctr., Inst. of Fluid Sci., Tohoku Univ., 2-1-1 Katahira, Aoba, Sendai, 980-8577 Japan, hosseini@ceres.ifs.tohoku.ac.jp), Takayuki Hirano, Osamu Onodera, and Kazuyoshi Takayama (Tohoku Univ., Sendai, Japan)

This paper reports on the application of underwater shock waves and vapor cavities for cerebral thrombolysis. The energy source was a $Q$-switched Ho:YAG laser (Nippon Infrared Industries Co., Ltd.) with 91 and $140 \mathrm{~mJ} /$ pulse energy measured at the end of a 0.60 -mm-diam glass optical fiber, pulse duration of $200 \mathrm{~ns}$, and wavelength of $2100 \mathrm{~nm}$. The whole sequences of the shock-wave propagation and growth of the cavitation bubbles from the end of the optical fiber were visualized by highspeed photography using an Imacon 468 camera (Hadland Photonics Ltd.). The shock-wave generation was associated with the laser breakdown in the water. The laser interaction produced a microplasma and heated the liquid in front of the fiber. The plasma drove spherical shock wave in water, followed by the formation of a high-temperature vapor cavity. Collapse of the cavity produced a secondary spherical shock wave in water. Successive generation and collapse of the bubbles were visualized. Pressure histories were measured by PVDF needle hydrophones. The underwater shock waves and the vapor cavities were successfully applied for disruption of artificial thrombosis. The result clearly shows potential extension of this method to precise medical treatment for revascularization in neurosurgery.

\section{$4: 20$}

2pBB9. Radiation and ultrasound-triggered bubble contrast agents based on superheated drops. Robert Apfel (Yale Univ., New Haven, CT 06520-8286)

Superheated drop compositions have been formulated for infusion in the body. Low doses of $\mathrm{x}$ rays or diagnostic ultrasound pulses are sufficient to nucleate the vaporization of these drops, producing bubbles that can serve as ultrasound contrast agents for imaging. Drugs can be carried in the drop material and can be released at a specific site where the radiation or ultrasound is focused. The current work describes the status of: (1) the optimization of the components that make up the composition, which include the superheated material, another liquid to adjust the degree of superheated, an encapsulant, and possibly a drug; (2) tests of the sensitivity of these drops to being triggered either by $\mathrm{x}$ rays or sufficiently intense pulses of ultrasound; (3) the analytic tools for characterizing the compositions and their triggering; and (4) the practical challenges to making this system suitable for clinical application.

\section{$4: 35$}

2pBB10. Effect of dual-reflector lithotripter on stone fragmentation and cell damage. Dahlia L. Sokolov, Michael R. Bailey, and Lawrence A. Crum (Appl. Phys. Lab., 1013 NE 40th St., Seattle, WA 98105)

An electrohydraulic lithotripter uses an ellipsoidal reflector to focus shock waves on to a kidney stone. The shock wave generates a cylindrical cavitation field, $\sim 1 \mathrm{~cm}$ wide $\times 10 \mathrm{~cm}$ long, that has been implicated in both stone fragmentation and damage to healthy tissue during lithotripsy treatment. A dual-reflector lithotripter, consisting of two identical sparkgap lithotripters facing each other and firing simultaneously, creates a more localized cavitation field, $\sim 3 \mathrm{~cm}$ wide $\times 5 \mathrm{~cm}$ long (Sokolov, Berlin 1999). Such a field may increase the rate of stone fragmentation while mitigating damage to surrounding tissue. Breakage of model stones at the focus and hemolysis of red blood cells $3 \mathrm{~cm}$ from the focus were assessed for both conventional lithotripsy (CL) and dual-reflector lithotripsy (DRL). To equalize total energy input, the number of shots was halved from CL to DRL. Stones subjected to DRL were broken into several major fragments while stones subjected to CL remained intact except for some dust. Human blood was diluted to $3 \%$ hematocrit in degassed PBS and placed in acoustically transparent sample tubes. There was no statistical difference in percent hemolysis between CL $(5.12 \pm 1.01 \%)$ and DRL $(5.39 \pm 0.57 \%)$. [Work supported by NIH DK43881 and NSF.]

\section{$4: 50$}

2pBB11. Cavitation enhanced acoustic hyperthermia. Patrick Edson, R. Glynn Holt, and Ronald A. Roy (Dept. of Aerosp. and Mech. Eng., Boston Univ., Boston, MA 02215)

The dominant mechanisms through which cavitation activity may lead to enhanced heating in tissue and tissue-like media with high-intensity focused ultrasound remain unclear. Previously, the authors have reported the results of experimental [J. Acoust. Soc. Am. 104 (A) (1998)] and numerical [ibid. 106 (A) (1999)] investigations which sought to understand the relationship between parameters relevant to the cavitation field, dominant heating mechanisms, and the magnitude of the enhanced heating effect. The model has since been extended to better account for heating from acoustic re-radiation. Results of expanded numerical simulations which incorporate bubble related heating source terms in an existing FDTD code designed to calculate temperature response as a function of the incident acoustic field are reported. Experiments aimed at manipulating the cavitation field to facilitate reproducibility and control are also reported. [Work supported by DARPA.]

\section{5:05}

2pBB12. Hepatic vasculature flow monitoring through the ultrasonic interruption of contrast agents. R. T. Rhee, J. B. Fowlkes, J. M. Rubin, and P. L. Carson (Dept. of Radiol., Univ. of Michigan Medical Ctr., Ann Arbor, MI 48109)

A technique has been developed to assess differential liver perfusion due to hepatic artery versus portal vein. This technique uses a continuous intravenous infusion of contrast agent and transcutaneous ultrasound to control the flow of contrast agent in selected vessels. A 1-in diameter transducer controlled the flow of perfluorocarbon-filled contrast agent in the abdominal aorta. It has been previously shown that acoustic amplitudes required to manipulate contrast agent are well within FDA limits. Pulsed ultrasound 10-cycle tonebursts at $2.25 \mathrm{MHz}$ and $2.7 \%$ duty cycle were applied to the abdominal aorta for $30 \mathrm{sec}$ to clear contrast agent from the hepatic artery, portal vein and liver parenchyma. The interruption transducer was then turned off, creating a sharp leading edge of contrast 
agent, with risetimes of approximately $0.1 \mathrm{sec}$ measured in the hepatic artery. Harmonic grayscale and spectral Doppler images were obtained on a Toshiba Powervision 8000 . The sharp contrast agent leading edge allows differential measurements of hepatic artery and portal vein arrival times. This time differential was measured at approximately $10 \mathrm{sec}$. These results indicate that image-guided insonification of the abdominal aorta may substantially improve discrimination of the hepatic arterial and portal venous supply in hepatic imaging.

\section{5:20}

2pBB13. Volume flow measurement using Doppler RF decorrelation. Theresa A. Tuthill, J. Brian Fowlkes, Jonathan M. Rubin (Dept. of Radiol., Univ. of Michigan Medical Ctr., Ann Arbor, MI 48109, tuthill@umich.edu), and Anne L. Hall (General Electric Medical Systems, Milwaukee, WI)

A technique for monitoring volumetric flow was developed by combining standard Doppler measurements with amplitude decorrelation. The proposed algorithm uses current clinical ultrasound technology and is independent of vessel orientation. However, to accurately measure the ultrasound signals' temporal decorrelation in blood, a contrast agent was added to increase the scattering signal strength. The algorithm uses standard frequency shifted Doppler signals, steered at two different angles, to determine the in-plane velocities. For the same firing lines, the corresponding temporal amplitude decorrelation is related to total velocity. The velocity normal to the imaging plane can then be extracted and integrated to compute total volume flow. The algorithm was tested in a flow tank using a GE Logiq 700 clinical scanner with a $7.5 \mathrm{MHz}$ linear array. The output power was set at the lowest level to reduce effects from additional decorrelation due to acoustic radiation force. RF data were collected as in-phase and quadrature components. The tube flow results showed a parabolic velocity profile that scaled with volume flow. For transducer angles close to perpendicular to the vessel, the average calculated volume flow error was less than $25 \%$. These preliminary results demonstrate the efficacy of our technique in measuring blood volume flow.

\section{$5: 35$}

2pBB14. The "underwater" sounds from the impact of drops in superfluid ${ }^{4}$ He. Antonios Apostolou (Grad. Prog. in Acoust., The Pennsylvania State Univ., State College, PA 16802), Jin H. So, Zhiqu Lu, J. Gladden, Eliza S. Bradley, and J. D. Maynard (The Pennsylvania State Univ., University Park, PA 16802)

When a drop of a liquid impacts onto a liquid surface a crater is formed, and the cavity dynamics that follow determine whether or not a bubble will be entrained. The entrainment of an air bubble following the impact of a raindrop onto the sea provides the predominant mechanism for the production of the underwater noise from rain. Experiments in water have indicated one particular entrainment region in a dimensionless parameter-space. This talk describes an ongoing research effort to study the phenomenon on a more fundamental basis using, instead of water, the pure and highly characterized liquid helium. The impact of liquid helium drops on a liquid helium surface is monitored by employing acoustic and optical techniques. Bubble entrainment in superfluid ${ }^{4} \mathrm{He}$, as well as the transition to nonentrainment, are observed, and the collected data indicate a new entrainment region. In addition, high-speed images of the crater motion reveal novel crater dynamics for the nonentrainment case. [Research supported by National Science Foundation DMR 9801844 and the Office of Naval Research.]

\title{
Session 2pEA
}

\section{Engineering Acoustics: Acoustic Measurements and Materials Characterization}

\author{
R. Lowell Smith, Chair \\ 1749 Linbrook Drive, San Diego, California $92111-7112$
}

Chair's Introduction-1:10

\section{Contributed Papers}

1:15

2pEA1. Evolutionary strategy algorithm for a complete characterization of porous materials using a standing wave tube. Youssef Atalla, Raymond Panneton (GAUS, Mech. Eng. Dept., Univ. of Sherbrooke, QC J1K 2R1, Canada, Raymond.Panneton@gme.usherb.ca), and Franck Sgard (LASH/DGCB URA CNRS 1652, ENTPE, France)

A widely used model for describing the attenuation of an acoustical wave propagating in a rigid open-cell porous material is the JohnsonChampoux-Allard (JCA) model. This model is based on five macroscopic parameters describing the porous medium: flow resistivity, porosity, tortuosity, viscous and thermal characteristic lengths. Simultaneously with the development of direct methods for measuring these five parameters, defining and solving inverse problems based on an artificial intelligence approach appears to overcome some of the limitations of the direct methods. In this work, an application of the evolutionary strategy (ES) algorithm for the estimation of the five parameters of a porous material from simple acoustical measurements is presented. First, a number of numerical tests are performed and the results are used like a priori knowledge of how to set up an evolutionary algorithm to solve such a difficult problem in the shortest time. In the second step, the final setup of the evolutionary algorithm is applied for evaluation of the five parameters from experimental measurements. The method seems practical and promising since it is based on simple acoustical measurements and avoids using complicated and unreliable measurement setups.

\section{$1: 30$}

2pEA2. Comparison of techniques for measuring the acoustic properties of porous materials. C. Walter Beamer and Ralph T. Muehleisen (Dept. of Civil, Environ., and Architectural Eng., Univ. of Colorado, Boulder, CO 80309)

There is a large variety of methods for measuring the acoustic properties of porous materials. Of particular interest to many acoustics researchers are the characteristic impedance and propagating wave number of the porous materials. The standing wave impedance tube and twomicrophone method of measuring those properties have both become standards. A number of extensions of the two-microphone method have been developed. Song and Bolton recently published a transfer matrix method utilizing four microphones [J. Acoust. Soc. Am. 107, 1131-1152]. Comparisons of the tradeoffs and errors inherent in the major methods for the 
measurement of the acoustical properties of porous materials are presented. [Work supported by the Office of Naval Research.]

\section{$1: 45$}

2pEA3. Resonant acoustic measurement of the effective diffusion coefficient of gases through soil. Richard J. Schuhmann (Dept. of Civil \& Environ. Eng., Penn State Univ., 212 Sackett, State College, PA 16802, rxs34@psu.edu), Matthew V. Golden, and Steven L. Garrett (Penn State Univ., State College, PA 16804)

Recent interest has developed within the environmental community in measuring the effective diffusion coefficient $\left(D_{e}\right)$ of gases through soil. An electroacoustic system will be described that uses a plane-wave resonator and a phase-locked-loop automatic resonance frequency tracker. The gas analyzer is simple and can be built for about $\$ 100$ from readily available plumbing parts and inexpensive transducers. A soil core is fitted into the top of a valved sample chamber and the resonator below is charged with a pure "tracer"' gas (e.g., He, $\mathrm{SF}_{6}$ ), then the valve opened. The rate at which the tracer gas is replaced by air within the resonator is controlled by the soil's $D_{e}$. The resonant frequency is maintained by a phase-lockedloop that compares the signal from an electret microphone with a square wave reference generated by the same monolithic function generator (XR 2206) that excited an electrodynamic loudspeaker with a sinusoidal current. The resonator temperature is monitored using a monolithic IC temperature sensor (AD 592) providing an output current of $1 \mu \mathrm{A} / \mathrm{K}$. The mean molecular weight of the gas mixture in the resonator is directly determined in real-time from the ratio of the absolute temperature to the square of the fundamental acoustic resonance frequency. [Work supported by ONR.]

2:00

2pEA4. Relationship between the diffraction constants, the reciprocity parameter, and the mutual radiation impedance of a pair of compact radiators: An improved Pritchard approximation. Steven R. Baker (Phys. Dept., Code PH/Ba, Naval Postgrad. School, Monterey, CA 93943) and Clyde L. Scandrett (Naval Postgrad. School, Monterey, CA 93943)

By the principle of reciprocity, the mutual radiation impedance matrix of an acoustical array in a stationary fluid must be symmetric. However, use of the classical "Pritchard approximation" to compute array mutual (mechanical) radiation impedance $\left(\mathbf{Z}_{\text {mut }}=j \operatorname{Re}\left[\mathbf{Z}_{\mathrm{self}}\right] e^{-j k d} / k d ; e^{j \omega t}\right.$ assumed) [R. L. Pritchard, J. Acoust. Soc. Am. 32, 730-737 (1960)] leads to a matrix which is not symmetric for nonidentical radiators. Also, it is restricted to low frequencies $(k a \ll 1)$. By a simple application of the principle of reciprocity, it is shown that the mutual mechanical radiation impedance of a pair of reasonably compact radiators in an otherwise unbounded stationary fluid is expressible in terms of their (complex) diffraction constants $\mathbf{D}_{1}$ and $\mathbf{D}_{2}$ and the (complex) spherical wave reciprocity parameter $\mathbf{J}_{s}$ as $\mathbf{Z}_{\text {mut }}=S_{1} S_{2} \mathbf{D}_{1} \mathbf{D}_{2} / \mathbf{J}_{s}$, where $S$ is the radiator surface area, $\mathbf{J}_{s}=2 d / j \rho f, d$ is the separation between radiator acoustic centers, $\rho$ is the fluid density, and $f$ is the frequency. (For mutual acoustical radiation impedance, simply drop the factors $S_{1}$ and $S_{2}$.) For spherical radiators of radius $a, \mathbf{D}_{n}=e^{j k a_{n}} /\left(1+j k a_{n}\right)$. Note, this newly derived form for $\mathbf{Z}_{\text {mut }}$ is guaranteed to be reciprocal. Also, for small $k a$, it reduces to $S_{1} S_{2} / \mathbf{J}_{s}$, which is equivalent to the classical Pritchard approximation, corrected for nonidentical radiators.

\section{2:15}

2pEA5. Measurements of orifice impedance with grazing flow. Nolan S. Dickey, Ahmet Selamet (Dept. of Mech. Eng., The Ohio State Univ., Columbus, OH 43212, dickey.21@ osu.edu), and Mehmet S. Ciray (Arvin Industries, Inc., Columbus, IN)

Perforated interfaces are commonly used in silencers for automotive applications. In these silencers, the acoustic behavior of the perforations, typically described in terms of a perforate impedance, can have a significant effect on silencer performance. The perforate impedance, in turn, is affected by the mean flow in the silencer. The focus of the present study is mean flow tangential to the perforate face (grazing flow), which is often predominant. In most cases, the perforate impedance cannot be determined analytically, and therefore experimental data and empirical models are necessary. Yet, experimental data suitable for use in models of perforated tube silencers are relatively scarce. The objective of the present investigation is to measure the impedance of perforated plates subjected to grazing flow. A description is given of the experimental apparatus, which has been designed for measurements with flow conditions representative of those in an automotive system. Data are presented for plates with square-edged circular orifices of varied dimensions. The results are reduced in terms of scaling parameters suitable for use in empirical perforate models.

\section{$2: 30$}

2pEA6. Effects of nonuniform stress on the properties of lead zirconate titanate. Robert S. Janus, James M. Powers, Frank A. Tito, Mark B. Moffett, and Michael D. Jevnager (Naval Undersea Warfare Ctr., Newport, RI 02841, janusrs@npt.nuwc.navy.mil)

The variations in the piezoelectric constant, $d_{33}$, and the dielectric constant, $K_{33}{ }^{T}$, for Navy type III lead zirconate titanate piezoelectric ceramic under nonuniform compressive mechanical stress have been experimentally evaluated. Peak compressive stress levels as high as $290 \mathrm{MPa}$ (42 $\mathrm{kpsi}$ ) with electric field intensities up to $\pm 790 \mathrm{kVrms} / \mathrm{m}( \pm 20 \mathrm{Vrms} / \mathrm{mil})$ were applied to a stack of piezoelectric ceramic bars. The maximum $d_{33}$ and stress-averaged $K_{33}{ }^{T}$ values measured were $159 \%$ and $135 \%$ larger, respectively, than the corresponding catalog values specified at low field and without a mechanical bias. The electrical losses of the material measured under the above conditions are also reported. [Work supported by the Space and Naval Warfare Systems Command.]

$$
2: 45
$$

2pEA7. Equivalent circuit representation of Poisson-coupled electroacoustic modes. R. Lowell Smith (Consultant, 1749 Linbrook Dr., San Diego, CA 92111)

Mode-coupling phenomena have long been recognized as contributing to the detailed frequency-dependent behavior of electroacoustic materials and structures. Coupled modes have the effect of repelling each other from the positions in frequency space that they would occupy if the coupling interaction were not operative. Today, mode-coupling effects are most often addressed using finite-element analysis methods. This paper reinvestigates the Poisson (modes coupled via shared inertial mass) modecoupling issue for simple electroacoustic structures from another perspective. Beginning with the piezoelectric equations of state, equivalent-circuit models are developed. These are useful for interpreting standard forms of impedance/admittance spectroscopy data for a variety of practical experimental geometries. The models are applied to measurement data and their usefulness for performance simulations is illustrated.

\section{3:00}

2pEA8. Bragg scattering by subsurface cracks and grooves. Eugene J. Danicki (Polish Acad. of Sci. (IPPT), 21 Swietokrzyska Str., Warsaw, 00-049 Poland)

Bragg scattering of bulk waves from shallow periodic grooves has been proven experimentally to be an effective method for generation of Rayleigh waves in solids. To study this phenomenon, a double layer of alternately positioned periodic cracks is investigated. The system looks like two aligned half-spaces with periodic grooves: cracks correspond to the main grooves' faces; the mechanical connection of the grooves' side faces makes the difference between these systems. A null-space expansion of the wave field is proposed that minimizes the resulting stress. The convenient BIS expansion method is applied in this extensive numerical investigation. For wide cracks, a mode can propagate in the system that resembles the Rayleigh wave because cracks make the substrate surface almost stress free. The efficiency of generation of this mode is studied as a function of crack period and width, the distance between crack layers (corresponding to the groove depth), and the angle of incidence of either 


\title{
Session 2pED
}

\section{Education in Acoustics: Informal Education in Acoustics}

\author{
Thomas D. Rossing, Chair \\ Physics Department, Northern Illinois University, DeKalb, Illinois 60115 \\ Invited Papers
}

1:00

2pED1. Interactive exhibits illustrating wind instrument acoustics. D. Murray Campbell (Dept. of Phys. and Astron., Univ. of Edinburgh, Edinburgh EH9 3JZ, UK) and Arnold Myers (Edinburgh Univ. Collection of Historic Musical Instruments, Edinburgh EH8 9AH, UK)

The authors were recently involved in developing demonstration equipment for musical instrument museums. The aim of this project, funded by the European Union, was to provide hands-on exhibits which would allow the user to explore some of the basic science underlying the operation of string, wind, and percussion instruments. While it is fairly straightforward to devise exhibits in which the user can pluck a guitar string, strike a tubular bell, or bow a violin, considerations of hygiene and the requirement for skilled embouchure techniques make it impossible to expect members of the public to blow demonstration wind instruments. To overcome this limitation, exhibits have been constructed in which a clarinet and a trumpet are played using artificial mouths. The user can adjust a valve controlling the flow of air from a blower to the artificial mouth; a digital manometer monitors the mouth pressure. The note played can be changed by operating a piston on the trumpet, or a key on the clarinet. The signal from a microphone registering the sound output is fed to the sound card of a PC, and specially written software allows the user to examine the waveform and the frequency spectrum of the sound.

\section{$1: 30$}

2pED2. Music and science: Workshops for teachers in elementary schools. Uwe Hansen (Dept. of Phys., Indiana State Univ., Terre Haute, IN 47809)

A number of workshops have been conducted with support from ASA Technical Initiative funds. The emphasis in the workshops is on assisting teachers to use music as a vehicle to introduce science concepts. A monochord is constructed. Teachers are provided a tuning fork, a resonance tube, a spring, a "singing" tube, and material to construct a monochord. Concepts discussed include wave properties such as wavelength, frequency, amplitude, phase, and speed of propagation. In addition the connection to music is made by observing standing waves and relating frequency ratios to musical intervals. The teaching approach and workshop content will be illustrated in the presentation.

\section{2:00}

2pED3. Websites, video, and audio recordings for informal education in acoustics. Thomas D. Rossing (Phys. Dept., Northern Illinois Univ., DeKalb, IL 60115)

It is becoming increasingly easier to study acoustics (or any subject) outside the classroom walls. This is partly due to the ready availability of learning materials on the Internet, and video and audio recordings that lend themselves to home study and informal study. We discuss some of the materials that are now available for informal education in acoustics.

2pED4. Shaping the experience in Experience Music Project: Striking a balance between entertaining and educating in a popular music museum. James Fricke and Andrea Weatherhead (Experience Music Project, 2901 3rd Ave. \#400, Seattle, WA 98121)

After eight years of planning, the Experience Music Project opened in Seattle on June 23, 2000. The institution's press materials state that: EMP invites visitors to participate in a total sensory experience: see rare artifacts and memorabilia, hear musicians tell their stories, or play an instrument and create your own music. Learning about music has never been so much fun. Does the experience live up to the hype? EMP's opening exhibitions, activities, and public programs are described, public and critical reception is reviewed, and lessons learned in the first months of operation are shared. The presentation will focus on multimedia exhibit components and interactive exhibits, with special emphasis on the design, development, and objectives of the Sound Lab area, EMP's hands-on activity and learning center. 
2pED5. The CSULB Mobile Science Museum: An interactive science outreach tool. James N. McKibben (College of Natural Sci. and Mathematics, California State Univ. at Long Beach, 1250 Bellflower Blvd., Long Beach, CA 90840, mckibben@csulb.edu) and R. Dean Ayers (California State Univ. at Long Beach, Long Beach, CA 90840)

Since 1980, a converted 27-ft recreational vehicle has been visiting local schools filled with a variety of hands-on science exhibits and activities. The Mobile Science Museum (MSM) has recently undergone a major renovation, including adding a generator. The MSM is now self-contained with inside exhibits that focus on the physical sciences, including sound, light, electricity, etc. The MSM also carries five large tables for outside activities, including a seawater touch-tank containing local intertidal animals, optical illusions, fossils, bones, etc. The majority of the exhibits have been developed or adapted for the MSM and utilize oscilloscopes, oscillators, strobes, power supplies, fans, and other equipment. University students act as docents and serve as enthusiastic role models for our young visitors. It is a compliment to the interest generated by the MSM that many of the current volunteer docents received their first exposure to the program when the MSM visited their schools several years ago. The major funding for the program has come from the CSULB Alumni Association, the Long Beach Industry-Education Council, and other individual support. The requests for visits greatly exceed the current ability to meet them, but this will improve as additional funding becomes available.

Mobile Science Museum Visit

In conjunction with this session, the CSULB Mobile Science Museum will be on display Tuesday afternoon from 1:00 p.m. to 6:00 p.m. in the parking area of the hotel (near the North Tower).

\title{
Session 2pNSa
}

\author{
Noise, NOISE-CON and Architectural Acoustics: Environmental Noise Focused on Combined \\ Noise Sources \\ Paul D. Schomer, Cochair \\ U.S. Army Construction Engineering Research Laboratory, P.O. Box 9005, Champaign, Illinois 61826-9005 \\ Brigitte Schulte-Fortkamp, Cochair \\ Department of Physics/Acoustics, Oldenburg University, Oldenburg D-26111, Germany
}

Chair's Introduction-1:15

Invited Papers

$1: 20$

2pNSa1. How many meta-analyses fit on the head of a pin? Sanford Fidell (BBN Technologies, 21128 Vanowen St., Canoga Park, CA 91303)

Meta-analyses of social survey findings on source-specificity of annoyance indicate that the prevalence of annoyance attributable to aircraft noise may be somewhat greater than the prevalence of annoyance attributable to noise produced by surface traffic of similar cumulative level. Likewise, the prevalence of annoyance induced by impulsive noise may differ from that of general transportation noise of similar level. But how annoyed are people who live next to highways that happen to be located near runway ends by the combination of aircraft and surface traffic noise? How annoyed are people who live next to railroad tracks that pass by shooting ranges? One approach to modeling the total annoyance engendered by exposure to multiple noise sources has been to assume that the interactions among source-specific annoyances are negligible, so that total annoyance can be expressed as some form of summation of individual source-specific annoyances. This simplifying assumption may not always be warranted, nor is it often the case that social and physical measurements yield data precise enough to reliably distinguish among alternate summation hypotheses. Policy-related interpretations of the findings of studies of combined annoyance of noises of differing origin should thus be viewed with a healthy skepticism.

\section{$1: 40$}

2pNSa2. The acceptor principle in German regulations for environmental noise. Dieter Gottlob and Jens Ortscheid (Federal Environ. Agency, Bismarckplatz 1, 14193 Berlin, Germany)

According to the acceptor principle, environmental exposure shall be assessed from the point of view of the affected persons, i.e., exposures from various sources shall be evaluated as a whole. While the acceptor principle is well established in environmental fields such as air pollution, it is rarely applied in noise regulations. In general, the noise sources are assessed separately. Sometimes the existence of residual noise leads to reduced requirements for the specific noise source under consideration. Important obstacles against 
the practical application of the acceptor principle in some countries are inconsistent noise descriptors, measurement and prediction procedures for different sources, and different legal approaches to control noise. Furthermore, there are not enough social field studies available which deal systematically with the assessment of the exposure of several noise sources. Therefore, the proposed procedures for assessing situations with combined noise sources are still lacking scientific support, especially concerning the temporal and spatial distribution of noise sources. In this lecture, the current state of the enforcement of the acceptor principle in German noise regulations will be presented. Some discussions within the framework of the new European noise policy will be reported.

\section{2:00}

2pNSa3. Assessment of annoyance in a multisource environment. Truls Gjestland (SINTEF Telecom \& Informatics, Trondheim, Norway) and H. M. E. Miedema (TNO, Leiden, The Netherlands)

The annoyance caused by common community noise sources (road traffic noise, rail noise, aircraft noise, etc.) has been shown to be highly correlated with the energy-equivalent noise level, LEQ (or similar derivatives) from that source. In some cases LEQ is adjusted depending on the time of day (as in LDN) or depending on the nature of the source (e.g., impulsive noise). So-called dose-response curves showing the relationship between the noise level and the annoyance have been established for a number of different noise sources. This annoyance assessment method, however, is only valid for single noise sources. The authors present a method for assessment of annoyance in the presence of several noise sources. Each noise source is transformed into a common reference source by means of established dose-response curves, and then added on an energy basis.

2pNSa4. The importance of loudness functions in assessing noise annoyance. Paul Schomer (USA-CERL-CNN, P.O. Box 9005, Champaign, IL 61826-9005, schomer@uiuc.edu)

Outdoors, Schomer et al. have shown that loudness weighting according to ISO 226 can be used to great advantage as a direct substitute for A-weighting. That is, loudness-weighted sound exposure level (LLSEL) can be used as a direct substitute for A-weighted sound exposure level (ASEL). LLSEL, computed with a time constant commensurate with the time constant of the human hearing process, provides significantly better correlation with community response across different noise sources than does ASEL. Indoors, room noise criteria (RNC) curves have been developed using these same human hearing function considerations. The RNC curves and associated computational procedure successfully bridge the gap between the PNC curves of Beranek and the RC curves of Blazier. Taken together, this body of outdoor and indoor research results shows the great importance of the human hearing function in assessing noise annoyance.

\section{2:40-3:00 Break}

2pNSa5. How to define synergetic effects of combined noises. Brigitte Schulte-Fortkamp (Dept. of Phys./Acoust., Oldenburg Univ., 26111 Oldenburg, Germany, brigitte@ aku.physik.uni-oldenburg.de)

Evaluation of noise annoyance has to take into account the multidimensionality in a given social situation. Noise annoyance research needs an interdisciplinary procedure methodologically including acoustics, physics, psychology, and sociology. People's judgments depend on multiple factors like environmental area, noise source, characteristic of noises, number of noise events over a day, subjective experience with noises, the social situation, etc. Judging annoyance includes a wide range of variability, which is highly correlated with the variability of living conditions. Considering those contextual conditions of noise annoyance judgments is one important procedure regarding the measurement on annoyance toward noise of combined sources. Taking into account the knowledge on annoyance judgments relying on different subject-oriented conditions, a measurement is needed which refers to objective and subjective parameters. The combination of noise sources is for the judgment of annoyance as well as important subjective parameters. Moreover, the relationship of both defines the background for assessments. Based on an analysis of interviews concerning noises from different sources and on the literature [e.g., Fields et al. (1998), Hatfield and Job (1998)], a proposal for an approach in noise annoyance measurement will be presented.

2pNSa6. What can classical and fuzzy concepts contribute to the analysis of masking effects in environmental noise surveys? Dick Botteldooren, Andy Verkeyn (Dept. of Information Technol., Ghent Univ., St. Pietersnieuwstraat 41, 9000 Gent, Belgium, botteldo@intec.rug.ac.be), and Peter Lercher (Univ. of Innsbruck, Sonnenburgstrasse 16/I, A-6020 Innsbruck, Austria)

In laboratory research masking of one noise source by another is well studied. Field research based on noise surveys fails to show this effect clearly. Both the uncertainty in noise levels and other environmental variables and the fuzziness in annoyance contribute to the difficulty of the problem. Traditional statistical and fuzzy techniques were used to look for masking effects in a large environmental noise dataset gathered in Austria. The fuzzy analyses use a rule-based model that is constructed to predict noise annoyance. Specific attention is paid to rules that predict masking based on variables such as background noise level, distance to masker, direction of bedroom and living room windows, etc. 
2pNSa7. Development of line-based noise pollution reduction programs. Andrew Wearne, Richard Heggie (Richard Heggie Assoc., Level 2, 2 Lincoln St., Lane Cove, NSW, Australia 2066), Suzanne Stuart, and Donald MacLeod (Maunsell McIntyre, Milsons Point, NSW, Australia 2061)

This paper details the results of a unique Noise Pollution Reduction Program sponsored by the Rail Access Corporation of NSW, Australia. The program covers almost $200 \mathrm{~km}$ of rail line, located mostly within urban areas of Sydney. The initial stages of the project involved establishing a database of land sensitivities along the selected rail lines, defining all relevant rail noise sources and their characteristics and developing a suite of available noise abatement options and associated costs. The second stage of the project focused on detailed computer noise modeling of the rail lines, coupled with a site-specific field verification study involving all train types and other traffic variables. The third phase of the study involved prioritizing each line and different sections within each line through the development of a unique Community Noise Burden (CNB) index. This index involves assigning relative weightings per given section of line according to the noise components, number and type of dwellings affected, noise exceedance levels, etc. Following the priority ranking of each section of line, a Noise Pollution Reduction Program was then designed, incorporating consideration of community annoyance, cost per CNB improvement, practicality, safety and other benefits/disbenefits.
2pNSa8. A statistical method for the evaluation of discrete noise sources submerged in background. Alois Heiss (Bavarian State Ministry for Environ. Affairs, Munich, Germany) and Robin T. Harrison (Murphy \& Harrison, Inc., Claremont, CA 91711, AirBearZln@aol.com)

Sometimes it is necessary to assess the contribution of a discrete source to the total soundscape even when the overall level of the discrete source is submerged in the background noise. An example of such a situation is the quantification of annoyance from the noise of a factory in the presence of the suburban background of traffic noise, children, birds, etc. The traditional method of making such an assessment, computation and scaling of signal-to-noise ratios in 1/3-octave bands, is not available if spectra of both the discrete source and the background cannot be obtained. Also, this method requires the use of sophisticated instrumentation and time-consuming measurements. This paper describes a methodology which extracts adequate statistical information from stochastic signals to determine the confidence intervals of percentile levels. The Leq of a discrete source in a noisy background can be determined with known high resolution from easily obtained field measurements. The procedure does not depend on frequency analysis. The method is currently being expanded to enable evaluation of level distribution of the source. Basic theory that supports the method is presented and software that facilitates its use is described. An example of its application is given.

\title{
Session 2pNSb
}

\section{Noise and NOISE-CON: Construction Machinery Noise Limits and Means of Compliance}

\author{
Daniel R. Raichel, Chair \\ 532 Spencer Drive, Wykoff, New Jersey 07981
}

Chair's Introduction-1:15

Invited Papers

$1: 20$

2pNSb1. Recent development in European legislation concerning construction equipment. Volker K. P. Irmer (Umweltbundesamt, 14193 Berlin, Bismarckplatz 1, Germany, volker.irmer@uba.de)

European noise legislation since the 1970s consisted of directives on the approximation of the laws of the Member States in order to ensure the smooth functioning of the internal European market. In the late 1990s, the protection of human health and well-being started to play a more important role. A new EU directive on noise emission from equipment for use outdoors replacing former directives concerning construction machines is in force since its publication in the Official Journal of the European Communities on 3 July 2000. It lays down provisions concerning the marking of equipment with its guaranteed sound power level for about 60 types of outdoor equipment (construction machinery, hobby and garden equipment, and some others) and for about 20 of those noise limits in two steps. Noise limits are demanding but the current state of the art of noise reduction allows compliance without excessive additional costs. The current directive states that only equipment complying with these provisions may be placed on the European market that consists of 375 million citizens. The European Directive now must be transposed in national legislation; the provisions have to be applied from 3 January 2002.

1:40

2pNSb2. Railway construction in Hong Kong: How to make it work. Glenn H. Frommer (Mass Transit Railway Corp., MTR Tower, Kowloon Bay, Kowloon, Hong Kong SAR, PROC)

The Mass Transit Railway Corporation designs, constructs, and operates one of the world's most intensively used railway networks with 2.3 million passengers daily on $70 \mathrm{~km}$ of track. New railway construction is often undertaken in densely populated urban environments with high-rise developments surrounding works sites. For the Lantau and Airport Railway, operational in 1998, the Corporation had to remove $1000 \mathrm{~m}^{3}$ of granite $7 \mathrm{~m}$ from a high-rise residential estate for the construction of Lai King Station. A 
lightweight noise enclosure was erected but not without significant noise and political impacts. However, the station was successfully completed on time and within budget. Current extensive modifications to Mei Foo Station $15 \mathrm{~m}$ from residential towers require a heavyweight noise enclosure to include vehicular emergency service access. The construction method was developed to minimize noise impacts. The skeleton of the enclosure is now in place and there have been no significant environmental problems. This presentation will detail the following: the analysis methods used in determining noise impacts; the structure and training employed within contracts to obtain compliance to the noise specification; and the manner in which the noise impacts are monitored and controlled.

\section{2:00}

2pNSb3. Noise control for a vacuum excavator: A mutually beneficial solution. Erich Thalheimer (Central Artery/Tunnel Project, 185 Kneeland St., Boston, MA 02111, exthalhe@bigdig.com)

The Central Artery/Tunnel (CA/T) Project in Boston (the Big Dig) makes use of several vacuum excavator trucks (vac-trucks) in order to reduce accidental utility disruptions. Unfortunately, these vac-trucks generate very loud low-frequency noise due to their vacuum blowers which can cause nearby buildings to vibrate and has led to significant numbers of complaints from distressed abutters. In reaction, the CA/T Project undertook an intensive study to determine what mechanisms were producing the offensive low-frequency noise, and to devise mitigation measures to effectively control the low-frequency noise so as to allow the vac-trucks to continue working without causing noise complaints. Working cooperatively with the manufacturer (Guzzler) as well as with the contractor (Walton Systems), new and improved (Universal) silencers were retro-fitted to a test vac-truck and the improvements in low-frequency insertion loss were measured and documented. The new silencers effectively reduced the $40-125-\mathrm{Hz}$ third-octave band range noise by $10-15 \mathrm{~dB}$, thus approaching the noise control design goals selected to avoid causing nearby buildings to vibrate. This is a prime example of how cooperative efforts between vehicle manufacturers, construction contractors, and project officials can lead to mutually beneficial results.

$$
\text { 2:20 }
$$

2pNSb4. Noise monitoring, control, and compliance practices at several construction sites in Southern California. Douglas K. Eilar (Douglas Eilar \& Assoc., 321 N. Willowspring Dr., Encinitas, CA 92024)

Noise protection for residential neighborhoods, commercial properties, and wildlife habitats near construction sites is achieved to varying degrees within a maze of local, state, and federal regulations. Standards for a given site typically include noise limits for public nuisance under the municipal ordinance, but a project may also be subject to more restrictive requirements emanating from conditions of approval from a discretionary permit and the environmental review process. Sometimes, goals or provisions of the municipality's noise element to the general plan are a factor. Sometimes, only noise monitoring is required, for documentation or to determine if noise is a problem. For wildlife, federal or state agencies may have established limits applicable to habitats of certain sensitive, endangered, or threatened species, during nesting season. Noise limits for constructing a subway access in Los Angeles require measurement of maximum noise level (Lmax), while noise limits for grading a park in San Diego use hourly average sound level (Leq). Different limits apply to intermittent versus continuous noise, to different times of day, and to different land uses. Noise reduction may be attained by means such as sound-attenuation barriers, certification of equipment noise emission prior to use, or operational controls.

\section{2:40-3:00 Break}

2pNSb5. Survey of worldwide legislation on the control of construction noise. Kai Ming Li (Dept. of Mech. Eng., Hong Kong Polytechnic Univ., Hung Hom, Hong Kong)

The sustained economic growth in many countries of the world in the past few years has led to significant increases in the construction activities for building new homes, new infrastructures such as highways, and airports for cities. This construction work inevitably causes an increase in noise levels. Indeed, the problem of noise is one of the most important environmental issues in recent years. With increased exposure and the greater sensitivity of much of the population, the number of noise-related complaints has grown at an alarming rate over the past few years. Current legislation imposes a limit of acceptable noise levels, but seems ready to be tightened further in reflection of popular demand. This paper reports a recent survey on the worldwide legislation on the control of construction noise. The survey concentrates on different countries in the Pacific Rim, the USA, and a few countries in the European Union. Relevant guidelines proposed by such international organizations as the World Health Organization and the Organization for Economic Cooperation and Development are also reported.

2pNSb6. Legislation concerning construction noise in Germany. Eberhard Fischer-Sheikh Ali and Volker K. P. Irmer (Umweltbundesamt, Bismarckplatz 1, 14193 Berlin, Germany)

German legislation concerning construction noise started in 1965. It covers noise exposure in the neighborhood due to construction sites as well as noise emission from construction equipment. Noise exposure caused by construction sites may not exceed values specified in an administrative provision. These values differ for various types of residential areas and for different periods of the day. If they are, however, exceeded, the administration shall take the appropriate measures to limit the exposure. Noise emission from certain construction equipment is regulated by an ordinance; it contains noise limits that must be observed by the manufacturers placing equipment on the market and the obligation to label all equipment is specified with the sound power level guaranteed by the manufacturer. The limits depend on the type of equipment and its installed power. The Environmental Label (Blue Angel) is a voluntary product designation, it is one of the so-called soft instruments of environmental policy. The Blue Angel for construction equipment is awarded to machines that yield a noise emission 5-12 dB(A) less than noise limits of the ordinance mentioned above. 
2pNSb7. Noise and vibration control for the Los Angeles Metro Rail Construction Project. Thanh Luc (Parsons Engineering Science, 100 W. Walnut St., Pasadena, CA 91124)

Parsons Engineering Science has been retained by the Los Angeles Metropolitan Transportation Authority as the noise and vibration consultant for construction of the 17-mile-long Metro Rail subway system for a period of more than 10 years. Throughout various construction phases, short-term and long-term noise and vibration monitoring have been conducted at sensitive receptor sites such as residences, schools, hospitals, recording studios, businesses, historic buildings, and hotels, to measure both background and construction activities. Continuous noise and vibration data are collected remotely over a computer terminal, such that levels can be monitored on a moment's notice; our engineers can hear the actual sounds at the remote monitoring site from the office (15-20 miles away), and simultaneously receive decibel levels reported in real-time. Studies are conducted to determine the impacts of various construction activities, such as pile installation, and analyze the feasibility of various construction methodologies based on project noise and vibration compatibility criteria. Mitigation measures are developed to reduce impacts. Examples of mitigation measures included the use of noise curtain walls, noise equipment specifications, selection of construction methodologies, and various acoustical treatments for noise sources. Through the monitoring program and numerous studies, noise and vibration impacts have been successfully minimized.
2pNSb8. Demonstration hearing conservation program for construction workers in Washington State. Alice H. Suter (Alice Suter and Assoc., 575 Dogwood Way, Ashland, OR 97520)

Construction workers have traditionally been an "underserved" population in the area of noise control and hearing conservation. Approximately 500,000 of these workers are currently exposed to potentially hazardous levels of noise and little progress has been made to the U.S. to mitigate this exposure. A labor-management-professional team composed of members of the Washington State Building and Construction Trades Council, the Building Trades Labor Management Organization, the Center to Protect Workers' Rights, and professionals in industrial hygiene, audiology, and medicine, have developed a "common sense" hearing conservation program to serve construction workers and employers. Initiated as a demonstration program in the State of Washington, the program is tailored to the U.S. construction industry. It will include worker and supervisor training using construction-specific materials, training in the fundamentals of construction noise control, fitting and training in the use of hearing protection devices, audiometric surveillance, centralized record keeping, and the evaluation of program effectiveness. Special emphasis is being placed on the use of readily available noise control solutions as well as hearing protection devices that will not degrade communication and the perception of warning signals. Program plans are currently being finalized and training will be initiated in December of 2000.

\title{
Session 2pNSc
}

\section{Noise and NOISE-CON: Vehicle Noise and Vehicle Noise Tests}

\author{
Paul R. Donavan, Chair \\ General Motors Corporation, $N \&$ V Center, 8916 Cooley Lake Road, White Lake, Michigan 48386-4027
}

\section{Contributed Papers}

2pNSc1. Rolling noise ANC system: An experimental facility test. Federico Rossi (Laboratorio di Acustica, Universit di Perugia, Via G. Duranti 1/A-4, 06125 Perugia, Italy)

A new active system for reduction of rolling noise produced at tireground contact is proposed. The system is constituted by acoustical emitters installed on the car mudguard close to tire-ground point of contact. Active control is achieved by means of an electronic control unit which generates the control signal according to a digital error minimization technique. The active system has been tested on a stationary condition by means of an original experimental facility built at Acoustic Laboratory of the University of Perugia. The facility reproduces the acoustical emission of rolling noise employing a recorded noise source. Experimental tests show that the proposed active noise control system is particularly suitable for rolling noise low-frequency components attenuation. Application on a traveling car is actually going on.

\section{$1: 35$}

2pNSc2. High-temperature quiet-flow test facility. Jim R. Cummins, Jr., Jim B. Causey, and David Kapsos (Universal Silencer, P.O. Box 411, Stoughton, WI 53589, US@Universal-Silencer.com)

A high-temperature, quiet-flow test facility for the testing and evaluation of exhaust silencers and silencer materials has been developed. This facility is driven by a rotary positive displacement blower delivering up to $110 \mathrm{~m}^{3} / \mathrm{min}(4000 \mathrm{cfm})$ of airflow. This airflow is put through both a passive and active silencer to achieve minimal uncontrolled noise in the test stream. Controlled sound is injected into the airflow by a set of loudspeakers in a mixing/settling chamber. The airflow is heated up to $540{ }^{\circ} \mathrm{C}\left[1000^{\circ} \mathrm{F}\right]$ by a $144-\mathrm{kW}$ electric heater just prior to entering the test section. The test section is rated for a $10-\mathrm{cm}-(4 \mathrm{in}$.) diam flow path and up to $1.8-\mathrm{m}-(6 \mathrm{ft})$ long sample. The outlet of the test section exhausts outside the test building and a microphone measurement system measures the radiated sound power level. This facility allows tests to be run with flow, sound level and temperature controls to obtain in situ measurements of silencer performance including insertion loss.

\section{$1: 50$}

2pNSc3. Sound transmission characteristics of bulb seals. Junhong Park, Luc G. Mongeau, and Thomas Siegmund (School of Mech. Eng., Purdue Univ., 1077 Herrick Labs., West Lafayette, IN 47907-1077)

Sound transmission through door and window sealing systems is one important contributor to interior noise in vehicles. The noise generation mechanism involves the vibration of the seal due to the unsteady wall pressures associated with turbulent flow over the vehicle. Bulb seals are often used in cars, an application where the seals experience large deflection variations due to build variation between vehicles. The performance of a bulb as a sound barrier was the focus of this study. The vibration response of a bulb seal together with the radiated sound was modeled using a finite element method. Material properties for the elastomeric foams were measured and used in the FEM. Acoustic-structure interactions were considered and the noise generated on the quiescent side of the 
seal was calculated. Experimental data was obtained for several bulb seal specimens using a reverberant room test method. The radiated sound intensity was measured using an intensity probe. The seal wall vibration was measured using a laser vibrometer. The transmission loss of the bulb seal was calculated from the measured data. It was compared with the value from the numerical simulations. The effects of bulb seal design parameters such as density, elastic modulus, and damping ratio were investigated.

\section{2:05}

2pNSc4. Vehicle door seal modeler for wind noise. Yuksel Gur (Ford Res. Lab., P.O. Box 2053/MD3135-SRL, Dearborn, MI 48121, ygur@mail.ford.com)

Wind-noise performance of a passenger vehicle plays a significant role in the customer's perception of overall vehicle quality. Wind noise aerodynamically generated on the exterior surface of a vehicle can propagate to the vehicle interior through body panels, window glass, and the seal gap between the door and door opening panel. Experimental studies indicate that automotive door seal systems are generally a major contributor to the vehicle wind-noise performance. Traditionally, automotive door seals are designed using the "bread board" and other "build and test" procedures. Two new finite-element-based acoustic-structure analysis tools have been developed to evaluate the wind-noise performance of door sealing system. These validated CAE techniques allow the engineer to predict door seal system performance in sound transmission and aspiration leakage before the availability of prototype hardware. These methods combine the separate analysis capabilities of finite-element analysis for acoustics, rubber elasticity, steady-state harmonic viscoelasticity, and a unique modeling approach to perform a fully coupled structure/acoustic interaction analysis of door seal systems. These unique CAE methods, experimental validations, and implementation of these techniques on product design will be presented.

\section{2:20-2:35 Break \\ 2:35}

2pNSc5. Maglev-A super fast train. Areg Gharabegian (Parsons Engineering Science, Inc., 100 W. Walnut St., Pasadena, CA 92114, Areg@gharabegian@parsons.com)

Magnetic levitation (Maglev) is a technology where magnetic forces lift, propel, and guide a vehicle over a guideway. This configuration eliminates contact between vehicle and guideway and permits speeds of up to $300 \mathrm{mph}$. The Federal Railroad Administration is evaluating the feasibility of Maglev construction in seven cities. Parsons Engineering Science conducted noise and vibration studies for four of these cities. Use of magnetic levitation and propulsion minimizes noise from mechanical and moving parts; however, the benefit of low-noise emission diminishes as a vehicle operates at high speed. Aeroacoustic sources generally dominate noise levels at speeds of $120 \mathrm{mph}$ or greater. High-speed Maglev passby is characterized by high-noise levels and brief duration, which may startle people who are close to the alignment. Maglev systems generate groundborne vibration when a vehicle travels above the guideway due to the sudden on and off load of the vehicle and magnetic current. Maglev noise, vibration, and startling sources as well as impacts and mitigation measures will be presented. Available vibration data are based on German standards because there is a prototype Maglev train in operation in Germany. These standards and procedures that are used to calculate equivalent vibration levels in in./s will be presented.

\section{2:50}

2pNSc6. Relation between noise emission, friction and rolling resistance of car tires-Summary of final results. Ulf S. I. Sandberg (Swedish Natl. Rd. and Transport Res. Inst., SE-58195 Linkoping, Sweden, ulf.sandberg@vti.se) and Jerzy A. Ejsmont (Tech. Univ. of Gdansk, PL-80952 Gdansk, Poland)

A European Union directive with noise emission limits for noise from vehicle tires is underway. It has been suggested that tires should meet certain limits also with regard to skid resistance, assuming a conflict between requirements for low noise and high friction. It is important to study whether there is any conflict between requirements with respect to noise emission from tires and other important performance characteristics. Therefore, the authors conducted an extensive experimental study on this topic in 1997-1999. Tire/road noise was measured with the CPX method on three road surfaces $(8 \mathrm{~mm}$ medium-textured SMA, $16 \mathrm{~mm}$ smoothtextured DAC, and $12 \mathrm{~mm}$ rough-textured surface dressing) at two speeds. Noise was also measured with the drum method on three drum surfaces (replica ISO10844 surface, smooth-textured surface and rough-textured surface). Friction was measured with friction tester BV12, using optimum slip and locked wheel, at $70 \mathrm{~km} / \mathrm{h}$, on a smooth-textured DAC. Rolling resistance was measured on a laboratory drum facility, using a smooth safety walk surface and a rough-textured surface. Correlations, for 75 to 100 car tires, between all measured variables have been calculated. It is shown that there is no significant conflict between low noise and high friction, nor between low noise and low rolling resistance.

\section{3:05}

2pNSc7. The effect of damping of the disc brake squeal noise. Kihong Shin, Jae-Eung Oh, Wootaek Kim (Dept. of Automotive Eng., Hanyang Univ., 17 Hangdang-Dong, Seongdong-Gu, Seoul 133-791, South Korea), and Mike Brennan (Univ. of Southampton, Southampton SO17 1BJ, England)

A novel mathematical two-degree-of-freedom model is introduced to describe the dynamics of a disc brake system. The model is emphasized on the interacting dynamics between disc and pad components, and is investigated to understand the mechanisms of the disc brake squeal noise. Sensitivity analysis is studied to find unstable regions considered as the squeal state. Time signals are also collected from a set of first-order differential equations of the model. The signals are then used for phase space analysis to investigate the size of limit cycles. Both sensitivity analysis and phase space analysis are focused on the effect of damping of the system. The results show that damping treatment should be done on both disc and pad, whereas damping treatment has been done on the pad only in most practical situations.

\section{$3: 20$}

2pNSc8. An auditory model for the prediction of detection thresholds of impulsive sound events. Scott Amman, Mike Blommer, and Jeff Greenberg (Ford Motor Co., Sci. Res. Lab., 20000 Rotunda Dr., P.O. Box 2053, MD 2115, RM 3135, Dearborn, MI 48121)

Impulsive sound events such as squeaks and rattles experienced in a vehicle can be a major source of customer dissatisfaction. These events almost always occur in the presence of some sort of background noise (e.g., wind, road and powertrain noise). When complete elimination of the impulsive sound event is either not possible, or too costly, the same effect can be had by pushing the level of the sound below the detection threshold. This paper describes the development of an auditory model that has the ability to predict detection thresholds of impulsive sound events in the presence of noise. The model was initially developed using equal-energy exponentially damped sinusoids with center frequencies of 250,500, 1000, 2000, 4000 and $8000 \mathrm{~Hz}$ mixed with pink noise. It was then validated using four different squeak and rattle sounds each mixed with three background noises recorded in a vehicle (wind, smooth and rough road noise). An up-down Levitt procedure was used for threshold determination. Two subjects participated in the first experiment and six in the second. In general, the developed model was able to predict detection thresholds with an error that is on par with the subject-to-subject variability observed in the experiment. 


\title{
Session 2pNSd
}

\section{Noise and NOISE-CON: Power Plant Acoustics Round Table}

\author{
Robert A. Putnam, Chair \\ Siemens Westinghouse PC, MC 590, 4400 Alafaya Trail, Orlando, Florida 32826
}

\begin{abstract}
A panel of invited speakers from Session 2aNSb: Power Plant Noise Control and Prediction and Industrial Noise will discuss issues confronting the accurate determination of emissions and immissions from power plants. Panelists will introduce issues including prediction, noise control techniques, and measurements and encourage questions and comments from the audience.
\end{abstract}

\section{Session 2pSA}

\section{Structural Acoustics and Vibration, Signal Processing in Acoustics and Physical Acoustics: Diagnostics of Vibration and Noise in Structures II}

\author{
Scott D. Sommerfeldt, Chair \\ Department of Physics and Astronomy, Brigham Young University, Provo, Utah 84602-4673
}

\section{Invited Papers}

\author{
$1: 30$
}

2pSA1. Meanings of SVD and wave-vector filtering in the near-field acoustical holography using the inverse BEM. Jeong-Guon Ih (Dept. of Mech. Eng., Korea Adv. Inst. of Sci. and Technol., Science Town, Taejon 305-701, Korea) and Sung-Chon Kang (Daewoo Heavy Ind. Ltd., 6 Manseok-dong, Dong-ku, Inchon 401-010, Korea)

In the conformal near-field acoustical holography (NAH), using the boundary element method (BEM), the vibroacoustic information on the source surface can be indirectly reconstructed by utilizing the measured field pressure and the inverse transfer matrix. The involved vibroacoustic transfer matrix is generally ill conditioned and the reconstruction process should include the singular value decomposition (SVD) in order to solve the problem in the inverse procedure. The accuracy of the reconstructed field is deteriorated substantially due to the ill conditioning of the transfer matrix and the inevitable measurement noise of field pressure related to the nonpropagating wave components. In this study, the computational processes of SVD and wave-vector filtering in the BEM-based $\mathrm{NAH}$ are discussed and their physical meanings are investigated through a simulation example. The vibroacoustic transfer matrix decomposed by SVD permits an effective regularization of the source field: This is possible because the involved information in the transfer matrix can be separated into the radiation efficiency and the wave modes at the source and surface fields. In particular, it is clearly shown that the restored source image can be improved dramatically by adopting the wave-vector filter that suppresses the nonpropagating wave components appropriately that cause the deterioration of the reconstruction accuracy.

\section{2:00}

2pSA2. Moving frame acoustic holography and its possible applications in machine monitoring and diagnostics. Yang-Hann Kim and Soon-Hong Park (Ctr. for Noise and Vib. Control, Dept. of Mech. Eng., KAIST, Science Town, Taejon 305-701, Korea, yhkim@mail.kaist.ac.kr)

Moving frame acoustic holography has been successfully applied to predict sound pressure distribution on the surface of interest to us. It has been motivated to enlarge the aperture size of the hologram. In the meantime, however, a great deal of application has been found in what is related to making sound image with regard to time as well as frequency. The method is, in fact, analogous to a copy machine. We make the image of sound, instead of duplicating light image, which has to do with a copy machine. The theory [Y.-H. Park and S.-H. Kim, J. Acoust. Soc. Am. 104, 3179-3189 (1998)] shows that we can travel an array of microphones over the surface of which we want to have a sound/noise image. The theory also permits one to recover the sound image from the data obtained by the array microphone fixed on the ground. The sound image is the spatial characteristics of sound that will be observed by the microphone system attached to the sources. This paper reviews possible areas of applications for a train or subway, or a quality control system in a factory. 


\section{2:30}

2pSA3. Characterizing mechanical system integrity using structural surface intensity. Jeffrey C. Banks and Stephen A. Hambric (217 Appl. Sci. Bldg., Penn State Univ., University Park, PA 16802, jcb242@psu.edu)

Many structural intensity measurements have been made on simple beam and plate-like structures to determine source strengths and transmission paths. When analyzing more complex structures it is often difficult to compute the intensity throughout the depth of an element. Using a method developed by Pavic [G. Pavic, J. Sound Vib. 115, 405-422 (1987)], the structural surface intensity (SSI) is computed on a gearbox housing using an array of accelerometers and strain gauges. The focus of this research is to use SSI as a machinery fault indicator, with the objective of developing a method that is more sensitive than other conventional diagnostic methods. Experimental data were taken on a gearbox from the Mechanical Diagnostic Test Bed (MDTB) at the Penn State Applied Research Laboratory as the gearbox was run to failure. The changes in energy flow are analyzed and characterized to provide criteria for fault detection. Analysis of the data indicates that significant changes in structural surface intensity occur as geartooth faults develop and that SSI can be more sensitive than other traditional indicators. [This research was supported by the Multidisciplinary University Research Initiative for Integrated Predictive Diagnostics (Grant No. N00014-95-1-0461) sponsored by the Office of Naval Research.]

\section{2:45-3:00 Break}

3:00

2pSA4. Vibration reduction characteristics of an isolated concrete test support. Hooshang Khosrovani (Veneklasen Assoc., 1711 16th St., Santa Monica, CA 90404, hkhosrovani@ veneklasen-assoc.com)

Some testing and research facilities require extremely low vibration background environments. Specially designed, isolated concrete supports are built to provide the required levels of isolation. The vibration isolation characteristics for an isolated concrete support have been measured and results are compared to predicted values.

\section{$3: 15$}

2pSA5. Seismic wave attenuation coefficient for soils. Hooshang Khosrovani and Jose Ortega (Veneklasen Assoc., 1711 16th St., Santa Monica, CA 90404, hkhosrovani@veneklasen-assoc.com)

Several projects over the past years have provided the opportunity to measure the ground propagation properties of seismic waves. Data from a variety of vibration sources and soil types have been summarized in the form of the coefficient of attenuation versus frequency in the ranges $5-250 \mathrm{~Hz}$. Also included in the summary are data from the published literature. Although the data exhibit a wide scatter their availability will enhance the effort to predict induced ground vibration. This information may in turn be used in predicting noise and vibration levels in structures.

\section{3:30}

2pSA6. Visual engineering environment based on interchangeable virtual instrument for acoustic pressure field. Huancai $\mathrm{Lu}$ (Acoust. Soc. Am., Dept. of Instrumentation Sci. \& Eng., Zhejiang Univ., Hangzhou 310027, PROC, hclu@moi-lab2.zju.edu.cn)

Architecture and kernel technology of a visual engineering environment for an acoustic pressure field is presented. The system software is constructed based on the hierarchy architecture of virtual instrument software and COM technique in order to realize the flexible reconfiguration of an acoustic pressure field measuring system. The attribute models of measuring channels and sensors are established for instrument's and sensor's interchangeability. The locking critical section technique is used for the security of multitask intercurrent process and share relation database. These kernel techniques make acoustic pressure field measuring system easily upgraded (e.g., the function and scale of the measuring system can be extended for different test tasks), instruments or sensors can be interchanged without modifying the software source codes. Therefore maintenance of the acoustic pressure field measuring system is greatly reduced and the lifecycle is prolonged. In this visual engineering environment, the measuring data of acoustic pressure field can be controlled in real-time and expressed in table, curve, bar chart, contour, and virtual instrument. Simultaneously the data can be transmitted through an ODBC interface to another computational environment (e.g., MATLAB) or Internet client for further analysis.

\section{$3: 45$}

2pSA7. Discharge acoustic signal detection in insulating oil using the optical fiber Sagnac interferometer. Jongkil Lee (Mech. Education, Andong Natl. Univ., Andong 760-749, Korea), June-ho Lee (Ho Seo Univ., Korea), and Sang-joon Kim (KEPRI, Korea)

In this paper, an optical fiber sensor (OF sensor) utilizing the principal of the Sagnac interferometer was proposed to detect the discharge acoustic signals which generated from a needle-sphere electrode system in insulating oil. The performance of the OF sensor was checked by a sinusoidal calibration signal generated by a PZT actuator at $198 \mathrm{kHz}$. The detected discharge signals consisted of the acoustic signal and electrical noise. The noise signal could be removed by a digital low-pass filter. It was demonstrated that the OF sensor in this research had a possibility to detect the discharge acoustic signals in the power apparatus.

\section{4:00}

2pSA8. A crack localization system based on the spatio-temporal gradient method. Kenbu Teramoto (Saga Univ., 1-Honjo Saga-shi, 8408502 Japan)

For several decades, nondestructive tests, including $\mathrm{x}$ rays, gamma rays, radar, infrared thermography, and acoustic methods have been used not only for concrete structures but for other types of materials. Acoustic methods are the oldest and most widely used nondestructive testing. They are based on the propagation, and in some cases reflection, of elastic waves in solids or over their boundary surfaces. A well-known example is striking an object with a hammer and listening to variations in the "ringing" sound to detect the presence of internal voids, external cracks, or other defects. Usually, there are three techniques based on acoustic nondestructive tests sorted into the wave-velocity method, resonance methods, and echo methods. This paper proposes a fourth method with spatiotemporal analysis, which has two processes, as follows. In the observation, the resulting surface wave, its partial derivatives for $x, y$, and $t$, are calculated at several points. In the detection, the rank of the short-duration covariance matrix of time-series vector $\mathbf{f}\left[=\left(f f_{x} f_{y} f_{t}\right)^{T}\right]$ classifies the situation of the surface-wave field. Consequently, the proposed method can provide the wave front and the location of the cracks as the singularity in the sound field.

\section{$4: 15$}

2pSA9. Usage of Wigner-Ville distribution for vibroacoustical forced oscillation diagnostics of cracks. Leonid M. Gelman (Dept. of Nondestructive Testing, Natl. Tech. Univ. of Ukraine, 37, Peremogy Pr., Kiev, 252056, Ukraine) and Denis A. Adamenko (Natl. Tech. Univ. of Ukraine, Kiev, 252056, Ukraine)

For diagnostics of fatigue cracks using vibration and noise, the new low-frequency vibroacoustical method of forced oscillations with nonstationary excitation is considered. The usage of an advanced time-frequency signal-processing technique based on Wigner-Ville distribution is proposed and developed to improve the effectiveness of diagnostics. The simulation results are presented. 
2pSA10. Detection of fatigue cracks by wavelet decomposition of the nonstationary narrow-band acoustical signal. Nadejda I. Bouraou (Dept. of Orientation and Navigation Systems, Natl. Tech. Univ. of Ukraine, 37 Peremogy pr., Kiev, 03056, Ukraine, nadya@burau.inec.kiev.ua) and Pavel I. Marchuk (Natl. Tech. Univ. of Ukraine, Kiev, 03056, Ukraine)

This paper presents new results of the signal processing of the nonstationary narrow-band acoustical signal for vibroacoustical diagnosis of air- vibratory excitation with a variable central frequency at the engine running start. The above-mentioned excitation is considered to be a linearfrequency-modulated signal with amplitude modulation by a stationary random process. The cracked blade is considered to be piecewise-linear system. Assuming these preconditions, the nonstationary narrow-band acoustical signal, radiated by the blade, is simulated and processed by using the wavelet decomposition technique. Fault detection is carried out at the approximation of the last (fifth) level of decomposition. The influence of the crack size increasing on the considered level approximation is investigated.

\title{
Session 2pSC
}

\section{Speech Communication: Measuring and Modeling Speech and Voice (Poster Session)}

\author{
Bruce R. Gerratt, Chair \\ Division of Head and Neck Surgery, University of California, Los Angeles, 31-24 Rehab Center, \\ Los Angeles, California 90095-1794
}

\section{Contributed Papers}

\begin{abstract}
All posters will be on display from 2:00 p.m. to 5:00 p.m. To allow contributors an opportunity to see other posters, contributors of odd-numbered papers will be at their posters from 2:00 p.m. to 3:30 p.m. and contributors of even-numbered papers will be at their posters from 3:30 p.m. to 5:00 p.m.
\end{abstract}

2pSC1. Effects of oscillation of a mechanical hemilarynx model on mean transglottal pressures and flows. Fariborz Alipour (Dept. of Speech Pathol./Audiol., Univ. of Iowa, 334 WJSHC, Iowa City, IA 52242) and Ronald C. Scherer (Bowling Green State Univ., Bowling Green, OH 43403)

This study introduces a mechanical model of the larynx for investigating the aerodynamics of phonation. The model mimics the hemilarynx. The tracheal inlet section was rectangular $(2.5 \mathrm{~cm}$ by $2 \mathrm{~cm})$. The vocal fold was fabricated from hard plastic with an attached oscillating plunger. A speaker assembly and audio amplifier drove the plunger, mimicking one-dimensional vocal fold motion toward a flat wall. The glottal shape was rectangular. The glottal diameter was well specified or dynamically followed with a laser system. The air was sucked through the channel using a vacuum with controlled speed. Frequency and amplitude of the glottis were varied. The mean pressure and mean flow data were recorded. For steady flow conditions, the glottal gap ranged from 0.4 to $2.5 \mathrm{~mm}$. The pressure coefficient for steady flow had a range of 3.1-1.3 for Reynolds numbers between 500 and 5000. For oscillation conditions: (a) the frequency was varied from 75 to $150 \mathrm{~Hz}$ while the amplitude was held (almost) constant; and (b) the amplitude was varied for a fixed frequency of $100 \mathrm{~Hz}$. The results indicate that oscillation amplitude has a strong effect on the flow resistance of the glottis. [Work supported by NIDCD Grant No. R01 DC03566.]

2pSC2. Airflow patterns in hemilarynx models. Fariborz Alipour and Douglas Montequin (Dept. of Speech Pathol./Audiol., Univ. of Iowa, Iowa City, IA 52242)

Airflow patterns of a hemilarynx were simulated numerically by the method of computational fluid dynamics (CFD) and were investigated experimentally with a flow visualization method. In the numerical model, vocal fold motion was simulated by sinusoidal variations of the inferior and superior glottal widths at different phase conditions. The straight glottal wall alternated between converging and diverging shape, while the input airflow varied sinusoidally between zero and a maximum. The control variables were oscillatory frequency, Reynolds number, and phase difference of the inferior and superior sections used for glottal motion. Flow visualization was performed using a hemilarynx physical model with a rectangular-shaped glottis. Using a Kodak high-speed camera, airflow patterns were recorded for various glottal widths and flow rates. CFD simulation described the complete airflow pattern and velocity distribution which indicated the existence of a high-speed jet near the fixed wall, flow separation, and vortex formations behind the glottis. Flow visualization also revealed similar patterns of flow separation, near-wall jet, and vortices below the jet. [Work supported by NIDCD Grant No. R01 DC03566.]

2pSC3. Direct measurement of the glottal volume velocity waveform in an in vivo canine model. Andrew Verneuil, Jody Kreiman, Bruce R. Gerratt (Div. of Head and Neck Surgery, UCLA School of Medicine, 62-132 CHS, Los Angeles, CA 90095, verneuil@ucla.edu), Vijay Dhir (Univ. of California, Los Angeles, CA 90095), and Gerald Berke (UCLA School of Medicine, Los Angeles, CA 90095)

Phonation occurs when the potential pressure energy provided by the lungs is translated into the kinetic energy of a glottal air pulse by the vibrating vocal folds. The primary acoustic source energy is described by the amount of air passing the glottis (volume) and the instantaneous speed at which it is moving (velocity). Indirect methods of acoustic analysis have provided estimates of the volume-velocity waveform. This study provides direct measurements by applying a constant temperature anemometer and videostroboscopic glottic analysis in an in vivo canine model. The velocity profile across the glottis and glottal area were measured in the anterior, middle and posterior glottis. These measurements were then temporally synchronized and combined to create a volumevelocity waveform of the acoustic source. Consistent with previous studies, velocity waveforms were double peaked across the glottis and were 
usually greatest anteriorly. Significant contributions to the volumevelocity waveform were made by the anterior and middle portions of the vibrating vocal folds, while the posterior glottis had less contribution. Inertial delays and flow reversals were demonstrated during the closed phase of phonation. Understanding the measured volume-velocity waveform provides more insight into the acoustic source spectrum and its role in phonation.

2 pSC4. Flow visualization in a model of the glottis with a symmetric and oblique angle. Daoud Shinwari, Ronald C. Scherer (Dept. of Commun. Disord., 200 Health Ctr., Bowling Green State Univ., Bowling Green, OH 43403, ronalds@bgnet.bgsu.edu), Abdollah A. Afjey, and Kenneth DeWitt (Univ. of Toledo, Toledo, OH 43606-3390)

Modeling the human larynx can provide insights into the nature of flow within the glottis. This study reports intraglottal pressure profiles and flow visualization for a symmetric and an oblique glottis with a glottal angle of 10 deg divergence. For the oblique case, the glottis slanted at an angle of $15 \mathrm{deg}$. A Plexiglas model of the larynx was used. Each vocal fold had at least 11 pressure taps. The minimal glottal diameter was held constant at $0.04 \mathrm{~cm}$. Each case was subjected to steady airflow corresponding to transglottal pressure drops of $3,5,10$, and $15 \mathrm{~cm} \mathrm{H}_{2} \mathrm{O}$. Pressure profile results showed that pressures were different on the two sides of the glottis; these data were strongly supported by an earlier study using a different model. Flow visualization in all cases showed that flow separated from one side of the glottis and remained attached to the other. For the oblique case, the separation point on the divergent wall moved upstream in the glottis with greater flows. The laminar core of the skewed jets decreased in length with higher flows. The jet caused asymmetric circulating regions downstream of the glottis in the reservoir section. [Work supported by NIH.]

2pSC5. Parameters for a first-order Kelvin model of laryngeal muscles. Eric J. Hunter and Ingo Titze (Dept. of Speech Pathol. and Audiol., Natl. Ctr. for Voice and Speech, The Univ. of Iowa, Iowa City, IA 52240, eric-hunter@uiowa.edu)

Because the intrinsic muscles of the larynx determine vocal fold posturing in phonation, a model of vocal fold posturing requires data from the passive and active viscoelastic properties of these intrinsic muscles. Using reported one-dimensional active (twitch and tetanus) and passive muscle (step elongation and relaxation) measures from fresh excised canine laryngeal muscles, one-dimensional first-order Kelvin model parameters were obtained. Obtaining these parameters was accomplished through optimization, where force-elongation patterns predicted by the model were compared to measured muscular passive cyclic force-elongation patterns. These parameters are reported along with the design for the Kelvin model. The results of this study, which will be integrated into a larger finiteelement posturing model, become the first step in predicting the vocal fold configurations needed for phonation.

2pSC6. New methods for objective evaluation of nasality. Masanobu Kumada (Dept. of Speech Physiol., Univ. of Tokyo, 7-3-1 Hongo, Bunkyo-ku, Tokyo, 113-0033 Japan), Toshiaki Kaneko (Sci. Univ. of Tokyo, Tokyo, 162-0825 Japan), Takahiko Ono, Masanao Ohashi, Koji Ishida (Ono Sokki, Yokohama, 226-8507 Japan), and Seiji Niimi (Univ. of Tokyo, Tokyo, Japan)

New methods for objective evaluation of nasality were introduced, including laser Doppler vibrometer, contact microphone system, and small microphone system. Subjects were two Japanese healthy males who speak Tokyo dialect. Tasks were sustained phonations of five Japanese vowels and $/ \mathrm{m} /$, repetitions of /ai/ and /mami/, and Japanese words of /shinksnsen/ and /shinbunshi/. Vibration of nasal skin was detected by laser Doppler vibrometer in terms of velocity $(\mathrm{Nv})$, and by contact microphone in terms of deviation $(N c)$. Vibration of neck skin on larynx was detected by contact microphone $(L c)$. Sound radiating from nares was detected by small microphone $(\mathrm{Nm})$. Sound radiating from nares and mouth was detected by standard microphone $(\mathrm{NOm})$. The accelerometer system was used as standard, consisting of two pickups, one of which detected vibration of nasal skin $(\mathrm{Na})$, and the other, neck skin on larynx $(L a) . N v / L c$, $\mathrm{Nv} / \mathrm{NOm}, \mathrm{Nc} / \mathrm{Lc}, \mathrm{Nm} / \mathrm{NOm}$ were compared with $\mathrm{Na} / \mathrm{La}$. These values were normalized as the value for sustained $/ \mathrm{m} /$ as 1.0 . Clinical use of these new methods was discussed.

2pSC7. Glottal characteristics of children-acoustic measures and comparison with adults. Helen M. Hanson, Kenneth N. Stevens (Sensimetrics Corp., 48 Grove St., Ste. 305, Somerville, MA 02144-2500, hanson@sens.com), and Ralph N. Ohde (Vanderbilt Univ., Nashville, TN 37212)

Previous studies of vowels produced by adult speakers showed substantial individual and gender differences in acoustic parameters related to glottal characteristics [H. M. Hanson, J. Acoust. Soc. Am. 101, 466-481 (1997); H. M. Hanson and E. S. Chuang, ibid. 106, 1064-1077 (1999)]. The current study extends the earlier work to children. In pilot work, data produced by seven children (five male, two female, ages 5-8) showing no signs of speech or voice disorders were analyzed. Acoustic measures related to first-formant bandwidth, open quotient, and spectral tilt were made on the vowel $/ \varepsilon /$ extracted from the word "red." These preliminary data show, for example, that the average measure of spectral tilt ( $\mathrm{dB}$ difference between amplitudes of first harmonic and third-formant prominence) for the children is about $14 \mathrm{~dB}$ greater than for adult females and about $24 \mathrm{~dB}$ greater than for adult males. The results suggest that, like adults, children display substantial individual differences in vocal-fold configuration. Data for additional subjects and vowels will be analyzed, and we will make measures related to aspiration noise. Mean data for children and adults will be compared and interpretation in terms of acoustic models will be proposed. [Work supported by NIH Grant DC03744.]

2pSC8. Utterance-final voice quality variations: Their perceptual structure and acoustic correlates. Rebecca Herman (Speech Res. Lab., Indiana Univ., 1101 E. 10th St., Bloomington, IN 47405, reherman@indiana.edu)

Voice quality variations can be used to mark prosodic and discourse boundaries in running speech. In order to quantify acoustic events in the signal during the production of these voice qualities, it is necessary to first discover their perceptual structure. The perceptual structure can then inform the acoustic analysis and help to find perceptually salient acoustic correlates. In this study, a perceptual experiment was run in which the stimuli were single words spoken by multiple talkers, which had all been extracted from the ends of utterances. Pairs of stimuli were given similarity ratings by listeners, and the similarity ratings were used in a multidimensional scaling analysis. The dimensions found in the multidimensional scaling analysis were interpreted using acoustic analysis. The voice qualities of the female voices in this study were found to have a primary perceptual split which was interpretable acoustically as laryngealized versus nonlaryngealized, but the voice qualities found in the male voices did not group into categories, and the perceptual dimensions were not as clearly interpretable in acoustic terms.

2pSC9. Phonetic targets as the link between speech production and speech perception. Yi Xu (Northwestern Univ., 2299 N. Campus Dr., Evanston, IL 60208) and Emily Q. Wang (Rush Univ., Chicago, IL 60612)

We argue in this paper that the coherence between speech perception and production, which the motor theory maintains, lies in the common knowledge about speech production shared by the speaker and the listener. More specifically, we propose that abstract phonemic units are associated with phonetic targets that are articulatorily operable. These targets, how- 
ever, are usually not fully achieved in speech production. Rather, they are often only approximated by different degrees under the limitation of various articulatory constraints. The process of speech production, therefore, is one in which various phonetic targets are being continuously approximated. As evidence, we will demonstrate with recent acoustic data how lexical tones in Mandarin are implemented through continuous approximation under specific articulatory constraints, how these constraints bring about extensive variations in the F0 contours, and how, despite the variations, tonal targets maintain their integrity in connected speech. We will then further argue that listeners, being also speakers themselves, should be able to discover the intended targets by noting the consistency the speaker manages to achieve despite the articulatory constraints. In other words, listeners hear not just what the speakers do, but more importantly, what they intend to do: We listen to hear what is being said.

2pSC10. Acoustic vowel space in 13 large Utah families. Kim CorbinLewis (Dept. of Communicative Disord. and Deaf Education, Utah State Univ., 1000 Old Main Hill, Logan, UT 84322-1000, KimC@ @oe.usu.edu), Julie M. Liss (Arizona State Univ., Tempe, AZ 870102), Hilary Coon (Univ. of Utah, Salt Lake City, UT 84113), and Steven D. Gray (Univ. of Utah Med. School, Salt Lake City, UT 84113)

Acoustic vowel space was examined in 131 speakers from 13 large Utah families ranging in age from 20-85 years with a median of 40 years. This population provides a unique opportunity to evaluate speech production characteristics in families with known genetic pedigrees and similar environmental influences. The purpose of the study was to determine whether acoustic manifestations of vowel production could be traceable to gender, age, or family membership. Results indicate that gender by itself explains $43.5 \%$ of the variance (highly significant, $p<0.0001$ ). Controlling for this gender effect, age is not a significant predictor of vowel space area $(p=0.29)$. However, being in the same family is a significant predictor of vowel space area $(p=0.002)$, independent of effects of gender and age. Being in the same family explains an additional $13.5 \%$ of the variance in area. [Work supported by NIH-NIDCD and the W. M. Keck Foundation.]

2pSC11. Individual differences in speech production: Voice-onsettime. J. Sean Allen and Joanne L. Miller (Dept. of Psych., 125 NI, Northeastern Univ., Boston, MA 02115, jallen@lynx.neu.edu)

As part of an ongoing study of talker-specific speech processing, we sought to document the existence of individual talker differences in voiceonset-time (VOT). Four female and four male talkers, all native speakers of American English, were asked to produce 30 repetitions each of 18 different words beginning with $/ \mathrm{p} /, / \mathrm{t} /$, or $/ \mathrm{k} /$. Words were displayed visually on a computer monitor one at a time in a randomized order at a constant presentation rate, and talkers were asked to produce the words at a comfortable speaking rate. The VOT and duration of each spoken word were measured. The eight talkers showed substantial individual variation in speaking rate (as measured by word duration) and, as would be expected, slower speaking rates were associated with longer VOT values. In order to determine whether there were individual differences in VOT beyond those associated with speaking rate, we conducted analyses that controlled for speaking rate on a word-by-word basis. These analyses revealed cases of consistent individual differences in VOT. We are currently examining whether the structure of listeners' perceptual voicing categories reflects such individual talker differences. [Work supported by NIH/ NIDCD.]

2pSC12. Perception of speaker characteristics with long and short samples. Blas G. Payri (LIMSI-CNRS, 91403 Orsay Cedex, France)

In this article, the perception of speaker's characteristics is compared using long and short samples. The sound material was an Italian read sentence (seicento-cinquantotto) and the last two syllables "to"' from this sentence. Twenty healthy Italian native speakers (10 females, 10 males) read the sentence, with the same recording conditions. In the first experiment, the listeners performed a free categorization of the syllables and then of the sentences. Then, the listeners were asked to give qualifiers to describe the classes they had made. The aim was to obtain the main criteria for the description of long samples (sentences) and short samples (isolated syllables). For the sentences, the gender and prosody criteria were more frequently used by listeners, whereas the pitch and voice quality were preferred to classify the syllables. In the second experiment, listeners rated the same samples as previously, along predefined axes (age, gender, breath, liveliness, etc.). The ratings were compared with acoustic measures. The results show that the ratings for the whole sentence, and for the extracted syllables, can be significantly different, leading to the conclusion that, within a sentence, there are significant local variations of the perceived voice quality and speaker characteristics as gender and age.

2pSC13. Effects of listener experience on measures of voice quality. Jody Kreiman, Bruce R. Gerratt (Div. of Head and Neck Surgery, UCLA School of Medicine, 31-24 Rehab. Ctr., Los Angeles, CA 90095-1794, jkreiman@ucla.edu), and Melissa Epstein (UCLA, Los Angeles, CA 90095)

Previous studies have shown that clinicians and naive listeners use different perceptual strategies when judging the quality of pathological voices. Naive listeners uniformly rely almost exclusively on $F 0$, while clinicians differ substantially from each other in the features they attend to. Consistent differences between groups in the overall level of rating have also emerged in paired comparison tasks, with naive listeners hearing greater differences between stimuli than clinicians do. We hypothesized that these differences in voice ratings are due to task-related factors, rather than to true differences in the ability of the two listener groups to hear differences in voice quality. Analysis-resynthesis approaches to quality measurement should eliminate such differences in measures of quality, because they require direct auditory matching of stimuli, without reference to internal standards that can be modified by auditory experience. To test this hypothesis, clinicians and naive listeners matched synthetic voice tokens to natural stimuli by adjusting synthesizer parameters of voice quality. No significant differences emerged between groups, suggesting that previously reported group differences are artifacts of traditional quality measurement tasks. Analysis-resynthesis approaches to measuring voice quality perception thus appear to control variability in judgments resulting from differences in internal standards. [Work supported by NIDCD Grant DC01797.]

2pSC14. The effects of speech rate and listening experience on the intelligibility of synthesized speech. Carol McCole (New York City Board of Education, Technology Solutions, District 75, 400 First Ave., New York, NY 10010 and Dept. of Speech, Commun. Sci., and Theatre, St. Johns Univ., Jamaica, NY 11439) and Fredericka Bell-Berti (St. Johns Univ., Jamaica, NY 11439)

Some persons with severe communication disorders are only able to communicate effectively using speech synthesizers, although even the most intelligible synthesis systems (e.g., DynaVox-DECTalk) have limitations. Listener success has been reported to vary with listener age, listener experience with synthesized speech, speech rate, and the presence of pauses between words. This series of experiments has examined the effects of listener age and experience, and speech rate and interword pauses on the perception of synthesized speech. Eighty IEEE-Harvard Sentences were presented to four groups of 12 listeners each [children (9-11 years), teens (14-16 years), young adults (20-25 years), and adults (38-45 years)], in two experiments. In the first experiment (40 sentences) speech rate was varied $(105,135,165$, and $195 \mathrm{wpm})$; in the second experiment (40 sentences), 7-12 days later, interword pauses occurred in half the sentences. Conditions were counterbalanced; no sentence was presented twice. Preliminary results indicate little effect of speech rate but a substantial effect of experience for the children: they improved substantially 
through experiment 1 ; the improvement carried over into experiment 2. Parallel results will be presented for all age groups. [Work supported by St. Johns University and New York City Board of Education, Technology Solutions, District 75.]

2pSC15. The role of speaker familiarity in assessment of dysarthric speech intelligibility. Kuo-You Huang (Dept. of Childhood Education and Nursery, Chia-Nan Univ., Tainan, Taiwan, ROC)

It is well known that dysarthric speech is often more intelligible to familiar listeners. The underlying mechanisms for this speaker familiarity effect, however, are not fully understood. The present study was conducted to investigate effects of speaker familiarity and laboratory training on perception of dysarthric speech. In the first experiment, a Chinesespeaking woman with cerebral palsy produced lists of bisyllabic words, pseudowords, and isolated monosyllables. Familiar and naive listeners transcribed these items. Results showed that familiar listeners yielded substantially higher intelligibility scores, and that the effect was more pronounced for meaningful words than pseudowords and monosyllables. It was shown that familiarity effect resulted from the interaction between speech decoding and word knowledge. In the second experiment, different intensive training programs were carried out to discover the dynamics of the familiarity effect: (1) feedbacks about the target words were or were not given to listeners; and (2) a forced-choice paradigm was applied in the training task and confusability among syllable pairs was manipulated, so that top-down and bottom-up influences could be differently observed during the training course. Results indicated that laboratory training was effective and it can, in fact, mimic the development of the familiarity effect in the naturalistic environment.

2pSC16. Intonation and emotion. Marc D. Pell (School of Commun. Sci. and Disord., McGill Univ., 1266 Pine Ave. W., Montreal, QC H3G 1A8, Canada, mpell@po-box.mcgill.ca)

Preliminary data were gathered on how simulated emotional characteristics of the voice influence the acoustic form of English utterances containing specific combinations of intonational and stress features [extending Eady and Cooper, J. Acoust. Soc. Am. 80, 402-415 (1986)]. Utterances varying in intrasentential focus position (initial, final, none) were elicited as both statements and questions in each of four emotional "modes" (neutral, sad, happy, angry) employing a structured elicitation procedure. Parameters of duration and fundamental frequency $(f 0)$ were then determined for the productions elicited from eight elderly speakers to specify important acoustic dimensions associated with specific combinations of stress, "modality," and emotional features of the stimuli. Results of the acoustic analyses largely reaffirmed past accounts of how contrastive focus is encoded in (affectively neutral) statements and questions for English, and cohered well with the acoustic literature on how basic emotions are expressed vocally for three key acoustic parameters (mean $f 0, f 0$ range, speech rate). The impact of emotion on linguistic attributes of prosodic structure was most evident in the speakers' modulation of $f 0$, which was notably constrained in prosodic conditions where speakers were required to signal "marked" emotional and nonemotional intentions conjointly within the intonation contour. [Work funded by FCAR.]
2pSC17. Eliciting speech styles in the laboratory: Assessment of a new experimental method. James D. Harnsberger, David B. Pisoni (Speech Res. Lab., Dept. of Psych., Indiana Univ., Bloomington, IN 47405, jharnsbe@indiana.edu), and Richard Wright (Univ. of Washington, Seattle, WA 98195)

This study concerned the development of a method of eliciting controlled stimulus materials, namely sentences, varying in three speech styles: reduced, citation, and hyperarticulated. Such a method would prove useful in studying the acoustics and perception of speech styles. The reduced style was elicited by having subjects read sentences while carrying out a distractor task that involved recalling a digit sequence from shortterm memory. The length of the sequence was calibrated to the individual subject via a digit span task administered prior to elicitation. It was hoped that the distractor task, serving as a cognitive load, would result in a less monitored, more naturalistic style. The citation style was elicited by having subjects read sentences from a list. The hyperarticulated style was elicited in a similar manner, except that subjects were prompted on some trials to reread the sentence again more carefully. Twelve subjects were recorded in this experiment, and the resulting sentences were acoustically analyzed and perceptually tested in a pairwise comparison task. The results showed that half of the talkers produced reduced sentences that differed acoustically and perceptually from citation sentences, while all 12 subjects produced hyperarticulated sentences that were differentiable from all others.

2pSC18. Direct classification of Thai monophthongs on two-dimensional acoustic-phonetic feature spaces in linear, mel, bark, and bark-difference frequency scales. Visarut Ahkuputra, Somchai Jitapunkul, Ekkarit Maneenoi (Digital Signal Processing Res. Lab., Dept. of Elec. Eng., Faculty of Eng., Chulalongkorn Univ.), and Sudaporn Luksaneeyanawin (Chulalongkorn Univ.)

A direct classification of nine Thai monophthongs was conducted on two-dimensional acoustic-phonetic feature spaces. Formant frequencies of a monophthong have been extracted from a stable vowel portion of a syllable nucleus. The $F 1$ and bark-difference $F 1-F 0$ represents tongue height. The $F 2$ and bark-difference $F 3-F 2$ represents tongue advancement. The Bayesian classifier utilizes statistical parameters, mean and standard deviation, of each monophthongs in classification. Twodimensional feature vectors comprise two acoustic-phonetic features, $F 2$ and $F 1$ in linear, mel, bark, and also, bark-difference $F 3-F 2$ and $F 1-F 0$. The classification results on the $F 2$ and $F 1$ space are $86.3325 \%$ in linear, $84.6187 \%$ in mel, $84.2331 \%$ in bark, $79.3916 \%$ in bark-difference $F 2-F 1$ and $F 1-F 0$, and also $79.9914 \%$ in bark-difference $F 3-F 2$ and $F 1-F 0$. Considering confusion matrices, the high vowels (/i:, v:, u:/), front-middle vowel (/e:/), and center-middle vowel (/q:/) have higher results than other groups which resulted from smaller overlapping and more separability between each group compared to low- and back-vowel groups. The back vowel (/u:, o:, @:/) has more misclassification between each monophthong than in other groups. Both feature spaces in bark-difference frequency scales exhibit lower-classification results although they illustrate better vowel distribution than other scales. 


\title{
Session 2pUW
}

\section{Underwater Acoustics and Acoustical Oceanography: High-Frequency Sediment Acoustics and Associated Sediment Properties and Processes II}

\author{
Kevin L. Williams, Chair \\ Applied Physical Laboratory, University of Washington, 1013 NE 40th Street, Seattle, Washington 98105 \\ Chair's Introduction-12:55
}

Contributed Papers

1:00

2pUW1. The scattering of low-grazing angle pulse beams at interfaces between fluid and porous media. Ralph A. Stephen (Woods Hole Oceanogr. Inst., Woods Hole, MA 02543)

Many environments in bottom interacting ocean acoustics consist of mud and sands which can be modeled as fluid saturated, porous solids with low shear moduli. To study the physical mechanisms responsible for forward- and backscattering in these media it is useful to have a forward modeling technique which applies to rough and heterogeneous porous bottoms. A numerical scattering chamber using the time-domain finitedifference method applied to the range-dependent Biot equations can be used to study the scattering of low-grazing angle pulse beams from fluidsaturated porous media. For example, for a flat seafloor over a homogeneous porous half-space, converted shear waves and converted compressional "slow" waves are shown to be excited in the subbottom even when the grazing angle is below the critical angle for compressional "fast" waves. Any scattering element near the seafloor will act as a secondary point source by Huygen's principle and when excited by an acoustic wave will have the potential to generate a family of interface waves and body waves of "fast" compressional, "slow" compressional and shear type.

$1: 15$

2pUW2. At-sea measurements of sound penetration into sediments using a buried vertical synthetic array. Harry J. Simpson, Brian H. Houston (Naval Res. Lab., Code 7136, 4555 Overlook Ave., Washington, DC 20375-5320), Alian R. Berdoz, Philip A. Frank, Steve W. Liskey (SFA, Inc., Largo, MD 20774-5322), J. S. Stanic (Stennis Space Center, MS 39529-5004), and Carl K. Frederickson (Univ. of Central Arkansas, Conway, AR 72035-0001)

At-sea measurements were conducted to understand the propagation of sound into sediments. These investigations used a new, buried vertical synthetic array system developed and built at the Naval Research Laboratory. The design of this at-sea system is based on a system developed for laboratory experiments that utilized a two-dimensional synthetic array technique [J. Acoust. Soc. Am. 107 (2000)]. For the measurements reported here, the hydrophone was water-jetted into the sediment to a depth of $2 \mathrm{~m}$. Once buried, the hydrophone was mounted to a vertical robotics stage that pulled the hydrophone up in $1-\mathrm{cm}$ increments. A spherical source, positioned $50 \mathrm{~cm}$ above the interface, was used to ensonify the sediment. The ensonification angle was varied by changing the horizontal distance of the source from the insertion point. In this manner, several measurements were made with ensonification angles above and below the critical angle. In addition, the enhancement of sound penetration due to interface roughness was investigated. This new system, along with the results of the measurements, will be discussed. [Work supported by ONR.]
$1: 30$

2pUW3. Spatial and temporal variation of large- and small-scale seafloor roughness. Kevin B. Briggs (Seafloor Sci. Branch, Naval Res. Lab., Stennis Space Center, MS 39529-5004)

Seafloor microtopography, measured at subcentimeter resolution over scales up to a meter or more by underwater stereo photogrammetry, was periodically characterized during the SAX99 high-frequency acoustic backscattering experiment. Analog 35-mm stereo photographs of seafloor roughness were digitized at 2- to 5-mm intervals from film transparencies with a stereo comparator capable of 1-mm-scale lateral precision and submillimeter-scale vertical accuracy. Relative seafloor height profiles were used to estimate roughness power spectra, which are parametrized as slopes and intercepts of regressions fit to the roughness power spectra. The composite roughness model developed by Applied Physics LaboratoryUniversity of Washington requires these two spectral parameters to determine the contribution of the seafloor roughness to the overall backscattering strength. Data from two locations over 31 days in the experimental area off Ft. Walton Beach, FL in 19-m water depth are presented in order to demonstrate the relationship between seafloor roughness morphology at subcentimeter to meter scales and model parameters predicting highfrequency acoustic scattering. Model predictions are generated from the measured geoacoustic properties of the rippled, medium sand seafloor and the range of roughness parameters that varied temporally and spatially during the experiment. [Work supported by ONR.]

\section{$1: 45$}

2pUW4. High-resolution measurements of volume heterogeneity in sandy sediments. Robert A. Wheatcroft, Cara E. Fritz, and Louise Hunt (College of Oceanic and Atmospheric Sci., Oregon State Univ., Corvallis, OR 97331)

As part of SAX 99, a multi-investigator study focused on the penetration and scattering of high-frequency $(10-300 \mathrm{kHz})$ sound in marine sediments, two independent measurements of sediment volume heterogeneity (SVH) were made. The first tool used to measure SVH was an in situ resistivity profiler (IRP) that logs sediment resistivity at 0.25 - $\mathrm{mm}$ vertical intervals to depths of $15 \mathrm{~cm}$. Robust empirical calibrations are used to calculate sediment porosity. The second method used to measure SVH was a digital $\mathrm{x}$-ray system that comprises a constant-potential x-ray source and an amorphous silicon x-ray detector. The IRP data indicate little between-site variability and bottom-type (i.e., ripple crest, ripple trough, mound) specificity. Profiles are characterized by a zone from 0 - to 3-mm subbottom of exponentially decreasing porosity, underlain by a zone of roughly constant porosity characterized by $5 \%-10 \%$ fluctuations. The digital $\mathrm{x}$ radiographs indicate three sources of SVH: (1) biogenic structures, (2) mud inclusions, and (3) shell fragments. Of these, the shell fragments are likely to be the most important sound scatterers. Estimates of shell fragment concentrations, distributions, and orientations will be discussed. [Work supported by ONR.] 
2pUW5. APL-UW environmental measurements during SAX99: Sediment conductivity and tomography. Dajun Tang, Kevin L. Williams, Darrell R. Jackson, and Eric I. Thorsos (Appl. Phys. Lab., Univ. of Washington, 1013 NE 40th St., Seattle, WA 98105)

Two new instruments were developed and deployed during SAX99 to measure surficial sediment variability at centimeter scales. Such data serve as input to acoustic models predicting sound scattering in the frequency range of $10-50 \mathrm{kHz}$. One instrument, IMP (In situ Measurement of Porosity) measures sediment conductivity at $1-\mathrm{cm}$ resolution in the horizontal dimensions and at 3-mm resolution in the depth dimension. From this instrument the following information is derived: (1) 3-D porosity or density variation in the top $12 \mathrm{~cm}$ of sediments, and (2) 2-D bottom roughness and associated spectra. The second instrument, the Acoustic Imager (AI), is a 3-D sediment tomographic tool with $1-\mathrm{cm}$ resolution operating at 170 $\mathrm{kHz}$. Information derived from the AI includes (1) 3-D sediment sound speed variability, (2) 3-D variability of sediment attenuation coefficients, (3) the presence and distribution of discrete scatterers such as shell pieces, and (4) the temporal variability of the above parameters over 3 days. These results and their implications to the acoustic measurements taken during the SAX99 experiment will be discussed. [Work supported by ONR.]

\section{2:15}

2pUW6. Quantification of Darcy's coefficient of permeability in siliciclastic marine sands using image analysis and effective medium theory modeling. Allen H. Reed and Dawn Lavoie (Naval Res. Lab., Bldg. 1005, Stennis Space Center, MS 39529)

Darcy's coefficient of permeability (Dcp) and porosity strongly influence the behavior of sound in unconsolidated sediments at high frequencies, therefore it is important to assess physical property values in sediments undisturbed by coring and subsequent handling. The objective of this study was to determine Dcp and porosity of siliciclastic marine sands from Ft. Walton Beach, FL that were embedded in situ using a polyester casting resin. For comparison, direct measurements of Dcp were made with an in situ falling head permeameter and porosity was determined by water weight loss. Modeling Dcp entailed quantifying pore dimensions and pore connectivity in the resin-embedded medium from twodimensional scanning electron microscopy images using standard image analysis techniques. The value of Dcp is estimated from average, or "effective," pore characteristics using an effective medium theory technique. Dcp of the samples in both vertical and horizontal directions averaged 1.9 $\times 10-3 \pm 1.3 \times 10-3$ and porosity averaged $0.44 \pm 0.04$. The modeled and measured Dcp values are comparable, whereas modeled porosity was slightly higher than measured porosity. From these data we conclude that estimating Dcp and porosity from in situ embedded sands results in values of physical properties close to those in the undisturbed state. [Work funded by ONR and NRL.]

\section{2:30-2:45 Break}

\section{2:45}

2pUW7. Estimation of seafloor roughness using ROV-mounted lasers. Brett K. McCurley, James N. Piper, and Nicholas P. Chotiros (Appl. Res. Labs., Univ. of Texas, Austin, P.O. Box 8029, Austin, TX 78713-8029, chotiros@arlut.utexas.edu)

The method employs lasers mounted on the remotely operated vehicle (ROV). Using a cylindrical lens to spread a laser beam into a fan and viewing the intersection of the laser fan with the water-sediment interface, it is possible to obtain a profile of the sediment. As the ROV advances, a bathymetric profile of the sediment may be constructed using successive profile snap shots. Data were collected at the Sediment Acoustics Experiment (SAX99) and processed to produce sediment surface profiles. [Work supported by the Office of Naval Research, Ocean Acoustics.]
2pUW8. Mechanisms for biological modification of seabed: Direct acoustic observations. D. V. Holliday, C. F. GreenlawIII, and D. E. McGehee (BAE Systems, 4669 Murphy Canyon Rd., Ste. 102, San Diego, CA 92123-4333)

Surface roughness and volume heterogeneity have both been implicated in the anomalous penetration of high-frequency sound into the ocean bottom. These physical characteristics of the seabed are dynamic properties of the bottom environment, with changes driven by both biotic and abiotic forces. Species that use the bottom for shelter from visual predators can change surface roughness at the time of emergence and re-entry. Acoustic observations of the movement of marine endo- and epibenthic organisms between the seabed and the water column will be used to illustrate the potential for these behaviors to modify the bottom. This behavior can also be the source of some of the dynamic volume heterogeneity that characterizes the seabed. The maintenance of burrows by animals is a known source of changes in both surface roughness and volume heterogeneity. At one study site fish appeared to anticipate the behavior of the plankton, foraging on lower trophic levels near, in, and on the seabed. This behavior can also modify surface roughness at the bottom-water column interface. [Research supported by ONR.]

\section{3:15}

2pUW9. Assessing 3D volume inhomogeneity using a noninvasive diver-deployed resistivity array. Peter D. Jackson, David A. Gunn (Br. Geological Survey, Keyworth, Nottingham NG12 5GG, UK), and Kevin B. Briggs (Naval Res. Lab., Stennis Space Center, MS 39529)

A noninvasive electrical resistivity array imaging system (RAIS) has been developed to measure sediment volume inhomogeneities in three dimensions. Novel measurements of electrical fields, due to controlled galvanic current-flow, have been inverted into electrical resistivities, in three dimensions beneath the seafloor. The limitations of conventional 3D resistivity inversions are explored, and additional a priori constraints are shown to significantly improve performance in the seafloor environment. From these $3 \mathrm{D}$ resistivity distributions, correlation lengths in $x, y$, and $z$ dimensions are calculated to support the modeling of acoustic scattering from the seafloor. Examples obtained during November 1999 in the Gulf of Mexico for the ONR High-Frequency Sound Interaction in Ocean Sediments (SAX99) experiment are described. New technology has reduced the acquisition time for a 3D dataset of 3840 measurements from approximately $2 \mathrm{~h}$ to $90 \mathrm{~s}$, enabling a suite of locations to be investigated during a single dive. Converting to formation factors and combining with porosity/density data enabled to tortuosity of the electrical current-paths to become a pore morphology descriptor. Formation factors were found in the range 2 to 5 , being consistent with substantial bioturbation in sandy sediments, with heterogeneities identified up to $100 \mathrm{~mm}$.

3:30

2pUW10. Imaging the permeability structure within near-surface sediments by acoustic crosswell tomography. Tokuo Yamamoto (Appl. Marine Phys. Div., RSMAS, Univ. of Miami, Miami, FL 33149)

Permeability structure within near-surface sediments is imaged by analytically inverting the measured fields of acoustic wave velocity and attenuation. The acoustic velocity and attenuation fields at $1000 \mathrm{~Hz}$ were previously measured by a pseudorandom binary sequence (PRBS) based crosswell acoustic tomography device [T. Yamamoto, J. Acoust. Soc. Am. 98, 2235-2248 (1995)]. A quadratic equation of permeability derived from the Biot theory is used for the permeability imaging. The porosity and shear modulus images are also extracted from the velocity and attenuation fields using the newly derived quadratic dispersion equation and an empirical shear modulus porosity depth of burial relation. The acoustically measured images of permeability, porosity, and shear modulus are favorably compared with the engineering tests performed on the cores and boreholes. 
$3: 45$

2pUW11. Ultrasonic studies on the effect of water content on compressional wave velocity in beach sand. Jacques $R$. Chamuel (Sonoquest Advanced Ultrason. Research, P.O. Box 81153, Wellesley Hills, MA 02481)

Theoretical results by Bachrach and Nur [Geophysics 63(4), 12251233 (1998)] indicate that the compressional wave velocity in wet sand decreases from 1700 to $250 \mathrm{~m} / \mathrm{s}$ as the moisture content is decreased by $1 \%$ from saturation based on the Biot-Gassmann theory low-frequency limit. New quantitative experimental results from beach sand $(280 \mu \mathrm{m}$ average grain size) are presented. Experimental results from wet sand "half-space" are compared with small wet sand samples. The sand moisture content was continuously measured from the weight of a mechanically balanced fixture holding ultrasonic transducers and the wet sand sample. Preliminary quantitative experimental results from the mechanically balanced wet sand show that the compressional wave velocity remained near $1650 \mathrm{~m} / \mathrm{s}$ in the $100-500 \mathrm{kHz}$ frequency range as the moisture content was decreased by more than $35 \%$ from saturation (typical drained sand is only about $5 \%$ from saturation). Beach sand, being angular with flat grain areas, may need to be modeled as platelets with large contact areas rather than spherical grains with point contact.

\section{4:00}

2pUW12. Effects of echo variability in geoacoustic sediment characterization. Daniel D. Sternlicht (ORINCON Corp., 9363 Towne Ctr. Dr., San Diego, CA 92121, dsternlicht@orincon.com) and Christian P. de Moustier (Scripps Inst. of Oceanogr., La Jolla, CA 92093-0205)

The variability in shape and amplitude of bottom echoes affects the resolution of geoacoustic parameter estimation techniques that match measured bottom-echo envelopes with envelope models derived from incoherent acoustic backscatter theory at the sediment-water interface and in the sediment volume. Following earlier echo envelope matching work carried out with data collected at $10-100 \mathrm{kHz}$ in San Diego Bay [Sternlicht and de Moustier, J. Acoust. Soc. Am. 105, 1206 (1999)], coarse- and fine-grain substrates are identified by estimates of their mean grain size $\left(M_{\phi}\right)$ and of the strength $\left(w_{2}\right)$ of their power-law roughness spectrum describing the spatial statistics of the interface. Statistics of the $\left(M_{\phi}, w_{2}\right)$ estimates are combined with those from Monte Carlo simulations, based on the data covariance matrices, to demonstrate that in sandy substrates, echo variability has little effect on estimates of $w_{2}$, and a pronounced effect on estimates of $M_{\phi}$. The reverse pattern is observed for fine-grain substrates. However, the parameter estimation technique used introduces a degree of correlation between $M_{\phi}$ and $w_{2}$, which is especially large for sand substrates. These observations are consistent with nature, where relief spectra of coarser sediments contain more energy than those of fine-grain substrates. [Work supported by ONR.]

\section{4:15}

2pUW13. Application of the Gelfand-Levitan method to geoacoustic inversion in shallow water. Kyle M. Becker (MIT/WHOI Joint Prog. in Oceanogr. and Oceanogr. Eng., Woods Hole Oceanogr. Inst., Woods Hole, MA 02543) and George V. Frisk (Woods Hole Oceanogr. Inst., Woods Hole, MA 02543)

The Gelfand-Levitan inverse technique is an exact inversion method originating in quantum scattering theory [I. M. Gelfand and B. M. Levitan, Am. Math. Soc. Transl. 1, 253-304 (1955)]. At a single frequency, the required input data are the Fourier transform of the plane wave reflection coefficient as a function of incident vertical wave number. The method was originally employed in underwater acoustics for a deep-water environment where a simple relationship exists between the reflection coefficient and the depth-dependent Green's function, which is obtained by Hankel transforming measurements of the point source pressure field [A. A. Merab, Sc.D. thesis, MIT/WHOI Joint Program, Woods Hole, MA (January 1987)]. In shallow water, the Green's function is characterized by poles corresponding to the eigenvalues of the perfectly trapped modes in the waveguide. These poles complicate the relationship between the Green's function and the reflection coefficient, and therefore the application of the Gelfand-Levitan technique. However, through application of the Darboux transform to the governing wave equation and the reflection coefficient, the Gelfand-Levitan method can be employed for shallowwater geoacoustic inversion [J. R. McLaughlin and S. Wang, in Mathematical and Numerical Aspects of Wave Propagation, edited by J. A. DeSanto (SIAM, Philadelphia, 1998), pp. 232-236]. In this talk, the Darboux transform will be illustrated and examples of geoacoustic inversion for synthetic shallow-water acoustic data will be presented. [Work supported by ONR.]

\section{$4: 30$}

2pUW14. Geoacoustic parameters by inversion of bottom reflectivity data from the Santa Barbara channel experiment. Ross Chapman and Danya Hudson (Ocean Acoust. Group, School of Earth and Ocean Sci., Univ. of Victoria, P.O. Box 3055, Victoria, BC V8W 3P6, Canada, chapman@uvic.ca)

A method is described to estimate the range dependence of geoacoustic parameters of the seafloor from bottom reflectivity data. The data were obtained using light bulbs as sound sources in the Santa Barbara channel experiment (SBCX). Light bulbs were deployed around the SBCX FFP array that consisted of five 30-element vertical line arrays. Signals from the direct path, the surface reflected path, and the four components of the first bottom reflection were clearly resolved at each array. The inversion integrates the results from several source-array combinations to generate estimates of the seafloor geoacoustic parameters in the area around the FFP array. The reflection coefficients were determined versus angle from the direct path and bottom-reflected path signals, and inverted using a simple fluid model to estimate the seafloor sound speed and density. The estimates are consistent with the known ground truth information for the SBCX site. [Work supported by ONR.]

\section{$4: 45$}

2pUW15. Sediment tomography in the East China Sea. Gopu R. Potty and James H. Miller (Dept. of Ocean Eng., Univ. of Rhode Island, Narragansett, RI 02882)

Sediment tomography experiments using broadband shots are planned as part of the Asian Seas International Acoustic Experiment (ASEAEX) in the East China Sea (ECS) in the year 2001. In order to gather adequate sediment information, gravity cores were taken from the experimental location during the spring of 2000. Using the gravity core data and available historic data, a sediment geoacoustic model for the region is presented. Synthetic experiments are performed with this geoacoustic model using broadband data. Various configurations of the explosive sources are tested to arrive at a suitable experimental geometry for the tomography experiment. This inversion is based on group speed dispersion of the broadband acoustic signal and is capable of estimating range-dependent sediment properties. [Work sponsored by ONR.] 